Check for updates

Cite this: RSC Adv., 2021, 11, 25700

Received 12th October 2020 Accepted 9th June 2021

DOI: 10.1039/d0ra08702e rsc.li/rsc-advances

\section{Novel mechanisms of the conformational transformations of the biologically important G. C nucleobase pairs in Watson-Crick, Hoogsteen and wobble configurations via the mutual rotations of the bases around the intermolecular $\mathrm{H}$-bonds: a QM/QTAIM study}

\begin{abstract}
Ol'ha O. Brovarets', (D) *a Alona Muradova ${ }^{b}$ and Dmytro M. Hovorun (D) ab
At the MP2/6-311++G(2df,pd)//B3LYP/6-311++G(d,p) level of quantum-mechanical theory, we provide for the first time a comprehensive investigation of the physico-chemical mechanisms of the 55 conformational transformations of the biologically-important G.C nucleobase pairs - Watson-Crick (WC), reverse Watson-Crick (rWC), Hoogsteen $(\mathrm{H})$, reverse Hoogsteen $(\mathrm{rH})$, wobble $(w)$ and reverse wobble (rw) base pairs by the participation of the $G$ and $C$ bases in the canonical and rare tautomeric forms (" $r$ " - means reverse configuration of the base pair). It was established that all these $G \cdot C$ nucleobase pairs can conformationally transform into each other without the changing of the tautomeric status of the $G$ and $\mathrm{C}$ bases. These transitions occur through significantly non-planar transition states via the mutual rotation of the $G$ and $C$ bases relative to each other within the $G \cdot C$ nucleobase pair around the upper, middle or lower intermolecular $\mathrm{H}$-bonds: $W C \leftrightarrow r W C, W C \leftrightarrow r W_{W C}, r W C \leftrightarrow W C, r W C \leftrightarrow W_{W C}, W_{W C} \leftrightarrow r W_{W C}, H$ $\leftrightarrow r \mathrm{H}, \mathrm{H} \leftrightarrow r w_{H}, r H \leftrightarrow H, r H \leftrightarrow W_{H}, W_{H} \leftrightarrow r w_{H}$. Gibbs free energies $\Delta G$ of activation for these conformational transformations are $\Delta G=2.96-19.04 / 3.58-13.36 \mathrm{kcal} \mathrm{mol}^{-1}$ (in vacuum under normal conditions $(T=298.15 \mathrm{~K})$ ), which means that these reactions proceed quite fast. Obtained conformational transformations are accompanied by the disruption and further formation of the intermolecular specific contacts in the G.C nucleobase pairs $(\mathrm{H}$-bonds and attractive van der Waals contacts). As a result, 76 conformers of the G.C nucleobase pairs were established - 48 base pairs in $W C, r W C, w_{W C}$ and $r w_{W C}$ configurations and 28 base pairs in $\mathrm{H}, \mathrm{rH}, \mathrm{w}_{\mathrm{H}}$ and $\mathrm{rw}_{\mathrm{H}}$ configurations with relative Gibbs free $\Delta G /$ electronic $\Delta E$ energies in the range $\Delta G / \Delta E=0.00-44.73 / 0.00-46.99$ and $\Delta G / \Delta E$ $=0.00-37.52 / 0.00-38.54 \mathrm{kcal} \mathrm{mol}^{-1}$, respectively (in vacuum under normal conditions). Experimental investigation and verification of the novel G.C nucleobase pairs are promising tasks for the future research. Based on the obtained data, biologically important conclusions were made about the importance of the conformational mobility of the G.C nucleobase pairs for the understanding of the functioning of the DNA and RNA molecules and their transition from the parallel into the anti-parallel duplexes and vice versa.
\end{abstract}

\section{Introduction}

In the classical conformational analysis of the nucleic acids, DNA and RNA, rotations of their structural fragments around the ordinary chemical bonds and bending of their rings, especially sugar residues, are considered as the source of their

\footnotetext{
${ }^{a}$ Department of Molecular and Quantum Biophysics, Institute of Molecular Biology and Genetics, National Academy of Sciences of Ukraine, 150 Akademika Zabolotnoho Street, 03680, Kyiv, Ukraine. E-mail: o.o.brovarets@gmail.com

${ }^{b}$ Department of Molecular Biotechnology and Bioinformatics, Institute of High Technologies, Taras Shevchenko National University of Kyiv, 2-h Akademika Hlushkova Avenue, 03022, Kyiv, Ukraine
}

structural variability. ${ }^{1}$ However, the pairs of the nucleotide bases, stabilized by the participation of the intermolecular $\mathrm{H}$ bonds, are usually considered as conformationally conservative, despite their soft structures. ${ }^{2,3}$ Their structural variability is usually associated with their insignificant deviations from planarity, which do not change the configuration of the base pairs. ${ }^{1}$ This structural variability can be induced by different external factors - stacking of neighboring pairs of bases, interaction with proteins, ligands of various nature and structure etc. (see ref. in work 1).

Shortly after the establishment of the spatial organization of the DNA molecule by James Watson and Francis Crick, the rare 
tautomeric hypothesis of spontaneous point mutagenesis was formulated, ${ }^{2,3}$ which considers the transformation or transition of the nucleotide bases from the main (canonical) into the rare (mutagenic) tautomeric forms due to the proton transfers as a main source of the origin of the spontaneous point mutations or structural variability. Since that time, the topic of prototropic tautomerism still remains important over decades to this day. ${ }^{4-14}$

In general, the topic of the prototropic tautomerism has actively attracted close attention in different areas of research drug design, proton transfer processes, formation of the mispairs, origin of the spontaneous point mutations, biologically important molecules and also play different significant roles in functioning of the DNA and RNA biomolecules. ${ }^{15-29}$ This fact enables to open new possibilities for the understanding of the fundamental mechanisms of the functioning of the biomolecules in a living cell.

The point of view, that tautomeric transformations in biological molecules are inseparable from the conformational transformations, is becoming more and more popular recently. ${ }^{30-36}$ Thus, lately it was found out, ${ }^{37-42}$ that intrapair proton transfer in the DNA base pairs causes their mutagenic tautomerization and conformational changes due to the mutual shifting of the bases respectively each other into the minor or major DNA grooves as well. These theoretical approaches have been realized for model objects, which, however, correctly reflects the real state of affairs and could be confirmed experimentally. ${ }^{42}$ Such approach significantly extends the biological importance of tautomerism and indicates that it goes far beyond the framework of the classical Watson-Crick tautomeric hypothesis, ${ }^{2,3}$ which considers tautomerism as the source of the origin of the spontaneous point mutations, which arise at the DNA replication.

As of today, while the conformationally-tautomeric mobility of the classical A-T DNA base pair has been already exhaustively explored, ${ }^{42-47}$ investigation of the conformational variety of the classical G.C DNA base pair has been insufficiently provided. Thus, recently it has been provided comprehensive research ${ }^{\mathbf{4 8 , 4 9}}$ of the tautomerization pathways of the reverse Löwdin $\mathrm{G}^{*} \cdot \mathrm{C} *(\mathrm{rWC})$, Hoogsteen $\mathrm{G}^{* \mathrm{t}} \cdot \mathrm{C}^{*}(\mathrm{H})$ and reverse Hoogsteen $\mathrm{G}^{* \mathrm{t}} \cdot \mathrm{C}^{*}(\mathrm{rH})$ nucleobase pairs by the participation of the bases in the rare, in particular mutagenic, tautomeric forms (marked with an asterisk "*”; "r" - reverse orientation of the base pair; " $\mathrm{t}$ " - trans-orientation of the $\mathrm{O} 6 \mathrm{H}$ hydroxyl group of the $\mathrm{G}$ base) via the single (SPT) or double (DPT) proton transfer along the neighboring intermolecular H-bonds with Gibbs free energies of activation for these reactions varying in the range 3.64$31.65 \mathrm{kcal} \mathrm{mol}^{-1}$ in vacuum under normal conditions $(T=$ $298.15 \mathrm{~K})$, leading to the novel $\mathrm{G} \cdot \mathrm{C}^{*}{ }_{\mathrm{O} 2}(\mathrm{rWC}), \mathrm{G}^{*}{ }_{\mathrm{N} 2} \cdot \mathrm{C}(\mathrm{rWC})$, $\mathrm{G}^{* \mathrm{t}}{ }_{\mathrm{N} 2} \cdot \mathrm{C}(\mathrm{rWC}), \mathrm{G}^{*}{ }_{\mathrm{N} 7} \cdot \mathrm{C}(\mathrm{H})$ and $\mathrm{G}^{* \mathrm{t}}{ }_{\mathrm{N} 7} \cdot \mathrm{C}(\mathrm{rH})$ conformers by the participation of the canonical and rare tautomers of the $\mathrm{G}$ and $\mathrm{C}$ bases. Further, these studies have been followed by our research, ${ }^{49}$ devoted to the physico-chemical mechanisms of the tautomeric wobblization of the biologically-important $\mathrm{G} \cdot \mathrm{C}$ nucleobase pairs - $\mathrm{G}^{*} \cdot \mathrm{C}^{*}(\mathrm{rWC}), \mathrm{G}^{* \mathrm{t}} \cdot \mathrm{C}^{*}(\mathrm{H})$ and $\mathrm{G}^{* \mathrm{t}} \cdot \mathrm{C}^{*}(\mathrm{rH})$, leading to the novel wobble $\mathrm{G} \cdot \mathrm{C}$ nucleobase pairs. However, these representations obviously could not explain the full range of the biological and structural properties of the nucleic acids, which continue to challenge by its mystery.

This work is intended to show at the example of the different biologically important G·C nucleobase pairs - classical Watson-Crick G·C(WC), reverse Watson-Crick G·C(rWC), Löwdin $\mathrm{G}^{*} \cdot \mathrm{C}^{*}(\mathrm{WC})$, reverse Löwdin $\mathrm{G}^{*} \cdot \mathrm{C}^{*}(\mathrm{rWC})$, Hoogsteen $\mathrm{G} \cdot \mathrm{C}(\mathrm{H})$ and reverse Hoogsteen $\mathrm{G} \cdot \mathrm{C}(\mathrm{rH})$, as well as wobble WatsonCrick $\mathrm{G} \cdot \mathrm{C}\left(\mathrm{w}_{\mathrm{WC}}\right)$, reverse wobble Watson-Crick $\mathrm{G} \cdot \mathrm{C}\left(\mathrm{rw}_{\mathrm{WC}}\right)$, wobble Löwdin $\mathrm{G}^{*} \cdot \mathrm{C}^{*}\left(\mathrm{w}_{\mathrm{WC}}\right)$, reverse wobble Löwdin $\mathrm{G}^{*} \cdot \mathrm{C}^{*}\left(\mathrm{rw}_{\mathrm{WC}}\right)$, wobble Hoogsteen $\mathrm{G} \cdot \mathrm{C}\left(\mathrm{w}_{\mathrm{H}}\right)$ and reverse wobble Hoogsteen $\mathrm{G} \cdot \mathrm{C}\left(\mathrm{rw}_{\mathrm{H}}\right)$ base pairs, which contain $\mathrm{G}$ and $\mathrm{C}$ bases in the main or rare tautomeric forms, that these base pairs are conformationally mobile structures and that rotations of the bases around the individual intermolecular $\mathrm{H}$-bonds are closely interconnected with the tautomeric status of the base pairs.

So, the aim of this study is to establish the conformational pathways for the different $\mathrm{G} \cdot \mathrm{C}$ nucleobase pairs through the mutual rotation of the $\mathrm{G}$ and $\mathrm{C}$ bases around the intermolecular H-bonds: WC $\leftrightarrow$ rWC, WC $\leftrightarrow \mathrm{rW}_{\mathrm{WC}}, \mathrm{rWC} \leftrightarrow \mathrm{WC}, \mathrm{rWC} \leftrightarrow \mathrm{W}_{\mathrm{WC}}$, $\mathrm{w}_{\mathrm{WC}} \leftrightarrow \mathrm{rw}_{\mathrm{WC}} ; \mathrm{H} \leftrightarrow \mathrm{rH}, \mathrm{H} \leftrightarrow \mathrm{rw}_{\mathrm{H}}, \mathrm{rH} \leftrightarrow \mathrm{H}, \mathrm{rH} \leftrightarrow \mathrm{w}_{\mathrm{H}}, \mathrm{w}_{\mathrm{H}} \leftrightarrow$ $\mathrm{rw}_{\mathrm{H}}$.

Such statement of the task is quite promising, since it enables to discover novel interpretations of the functional role of the so-called excited states - different conformers of the $\mathrm{G} \cdot \mathrm{C}$ nucleobase pair by the participation of the canonical and rare tautomers, which are now attributed to the biologicallyimportant functions, especially in the RNA molecule. ${ }^{15-20}$

As a results of this investigation, for the first time except the classical Watson-Crick G·C(WC) and Hoogsteen G·C(H) base pairs, it was revealed wide variety of the novel base pairs by the participation of the canonical and rare tautomers of the $\mathrm{G}$ and $\mathrm{C}$ bases and physico-chemical mechanisms of their mutual conformational transformations into each other. Altogether, it was discovered 76 conformations of the $\mathrm{G} \cdot \mathrm{C}$ nucleobase pairs 48 base pairs in $\mathrm{WC}, \mathrm{rWC}, \mathrm{w}_{\mathrm{WC}}$ and $\mathrm{rw}_{\mathrm{WC}}$ configurations with relative Gibbs free $\Delta G$ /electronic $\Delta E$ energies in the range $-\Delta G$ / $\Delta E=0.00-44.73 / 0.00-46.99 \mathrm{kcal} \mathrm{mol}^{-1}$, respectively, and 28 base pairs in $\mathrm{H}, \mathrm{rH}, \mathrm{w}_{\mathrm{H}}$ and $\mathrm{rw}_{\mathrm{H}}$ configurations with $\Delta G / \Delta E=$ $0.00-37.52 / 0.00-38.54 \mathrm{kcal} \mathrm{mol}^{-1}$, respectively, in vacuum under normal conditions $(T=298.15 \mathrm{~K})$.

Based on these data, it was expressed assumption about their possible biological role in the conformational transformations of the DNA and $\mathrm{RNA}^{\mathbf{1 5 - 2 0}}$ from parallel to antiparallel orientation and vice vers $a^{50}$ without proton transfer and changing of the tautomeric status of the nucleobases.

These obtained results extend the existing thoughts about the microstructural mechanisms of these processes, as well as about their functional role.

\section{Computational methods}

\section{Density functional theory calculations of the geometry and vibrational frequencies}

Equilibrium geometries of the investigated $\mathrm{G} \cdot \mathrm{C}$ nucleobase pairs and transition states (TSs) of their mutual conformational transformations, as well as their harmonic vibrational frequencies have been calculated at the B3LYP/6-311++G(d,p) 
level of theory, ${ }^{51-55}$ using Gaussian'09 program package. ${ }^{56}$ Applied B3LYP/6-311++G(d,p) level of theory has approved itself successfully for the calculations of the similar systems and processes and shown acceptable level of accuracy and adequacy of the obtained results. ${ }^{49,57,58}$ A scaling factor that is equal to 0.9668 has been applied in the present work for the correction of the harmonic frequencies for all complexes and TSs of their conformational transitions. ${ }^{\mathbf{4 4 , 4 5}}$

We have confirmed local minima and TSs, localized by Synchronous Transit-guided Quasi-Newton method, ${ }^{59}$ on the potential energy landscape by the absence or presence, respectively, of one imaginary frequency in the vibrational spectra of the complexes. All reaction pathways have been reliably confirmed by performing the optimization of the structures, which are close to the TS in the forward and reverse directions at the B3LYP/6-311++G(d,p) level of theory.

All calculations have been performed in the continuum with $\varepsilon=1$, that adequately reflects the processes occurring in real biological systems without deprivation of the structurallyfunctional properties of the bases in the composition of the DNA or RNA molecules and satisfactorily models the substantially hydrophobic recognition pocket of the DNA-polymerase machinery as a part of the replisome. ${ }^{\mathbf{6 0 , 6 1}}$

\section{Single point energy calculations}

We continued geometry optimizations with electronic energy calculations as single point calculations at MP2/6$311++\mathrm{G}(2 \mathrm{df}, \mathrm{pd})$ level of theory. ${ }^{\mathbf{6 2 , 6 3}}$

The Gibbs free energy $G$ for all structures was obtained in the following way:

$$
G=E_{\mathrm{el}}+E_{\mathrm{corr}}
$$

where $E_{\mathrm{el}}$ - electronic energy and $E_{\text {corr }}$ - thermal correction.

\section{QTAIM analysis}

Bader's quantum theory of Atoms in Molecules (QTAIM) ${ }^{64-68}$ was applied to analyse the electron density distribution, using program package AIMAll. ${ }^{69}$ The presence of the bond critical point $(\mathrm{BCP})$, namely the so-called $(3,-1) \mathrm{BCP}$, and a bond path between the donor and acceptor of the $\mathrm{H}$-bond or attractive van der Waals contact, as well as the positive value of the Laplacian at this BCP $(\Delta \rho>0)$, were considered as criteria for the formation of the $\mathrm{H}$-bond or attractive van der Waals contact. ${ }^{64-68}$ Wave functions were obtained at the B3LYP/6-311++G(d,p) level of theory, used for geometry optimisation.

The atomic numbering scheme for the DNA bases is conventional. ${ }^{36}$ In this study rare tautomeric forms of the $\mathrm{G}$ and $\mathrm{C}$ nucleobases are marked by an asterisk. ${ }^{\mathbf{1 0}}$

\section{Obtained results and their discussion}

Received results are presented on Table 1 and in Table 2. So, based on the obtained data, it would be firstly formulated and analysed basic results, which have been obtained for the first time and which have the closest relationship to the structural biology and molecular biophysics.

Altogether, it was revealed 62 novel G.C nucleobase pairs with wobble $(\mathrm{w})$ and reverse wobble (rw) geometries from the side of the Watson-Crick and Hoogsten edges $(\Delta G / \Delta E=0.00-$ 33.62/0.00-35.41 and 0.00-35.23/35.50 kcal mol${ }^{-1}$ ), respectively, which are planar and significantly non-planar pairs, stabilized by the participation of at least two H-bonds, one of which could be connected to the amino nitrogen atom of the $\mathrm{G}$ or $\mathrm{C}$ bases (Table 1). Such configuration of base pairs with necessity entails their tautomerization via the protonated amino group as transition state. However, they would become the subjects of our precise consideration in further investigations.

Altogether, for the $\mathrm{G} \cdot \mathrm{C}$ nucleobase pairs in the WatsonCrick (WC) and Hoogsteen $(\mathrm{H})$ configurations involving basic and rare tautomeric forms of the $\mathrm{G}$ and $\mathrm{C}$ bases, we have localized 55 transition states ( 34 for the $\mathrm{G} \cdot \mathrm{C}$ base pairs from the WC side and 21 for the $\mathrm{G} \cdot \mathrm{C}$ base pairs from the $\mathrm{H}$ side), defining the conformational transformations by the mutual rotations of the bases around the individual intermolecular $\mathrm{H}$-bonds (Tables 1 and 2). These conformational transformations of the $\mathrm{G} \cdot \mathrm{C}$ nucleobase pairs occur with or without the changing of the general geometry of the base pair and break of the intermolecular specific contacts with their further formation (H-bonds and van der Waals contacts), leading to the reorientation of the base pairs with cis-oriented $\mathrm{N} 1 \mathrm{H} / \mathrm{N} 9 \mathrm{H}$ glycosidic bonds to the base pairs with trans-oriented $\mathrm{N} 1 \mathrm{H} / \mathrm{N} 9 \mathrm{H}$ glycosidic bonds and vice versa: WC $\leftrightarrow$ rWC, WC $\leftrightarrow \mathrm{rw}_{\mathrm{WC}}, \mathrm{rWC} \leftrightarrow \mathrm{WC}, \mathrm{rWC} \leftrightarrow$ $\mathrm{w}_{\mathrm{WC}}, \mathrm{w}_{\mathrm{WC}} \leftrightarrow \mathrm{rw}_{\mathrm{WC}}, \mathrm{H} \leftrightarrow \mathrm{rH}, \mathrm{H} \leftrightarrow \mathrm{rw}_{\mathrm{H}}, \mathrm{rH} \leftrightarrow \mathrm{H}, \mathrm{rH} \leftrightarrow \mathrm{w}_{\mathrm{H}}, \mathrm{w}_{\mathrm{H}}$ $\leftrightarrow \mathrm{rw}_{\mathrm{H}}$ (Tables 1 and 2).

In those cases, when TSs are joined by single intermolecular $\mathrm{H}$-bond (Table 1), in most cases their lengths exceed the length of the analogical $\mathrm{H}$-bond at the starting base pair, which is stabilized by two anti-parallel $\mathrm{H}$-bonds. This fact clearly indicates that the latest are cooperative and mutually reinforce each other. By the way, this approach could be also applied for the numerical estimation of the cooperativity of the intermolecular H-bonds.

Characteristic feature of all TSs is quite low values of the imaginary frequencies $\nu_{\mathrm{i}}=11.2-101.9 / 11.3-104.7 \mathrm{~cm}^{-1}$ (Tables 1 and 2), which indicates the structural softness of these transformations, and relatively low values of their barriers $\left(\Delta \Delta G_{\mathrm{TS}} / \Delta \Delta E_{\mathrm{TS}}=2.96-19.04 / 3.58-13.36 \mathrm{kcal} \mathrm{mol} \mathrm{m}^{-1}\right.$ under normal conditions) ( $T=298.15 \mathrm{~K}$ ), pointing on the quite high speed of these conformational transformations. Just only these facts soundly testify that $\mathrm{G} \cdot \mathrm{C}$ pairs of nucleotide bases in the basic and rare tautomeric forms are conformationally mobile structures. It is quite evident that this biological observation can be transferred on the other pairs of nucleotide bases, independently from their nature and structure, almost without any quality restrictions.

It should be attracted attention to the unexpected results of this investigation and their comprehensive discussion (Tables 1 and 2).

1. It was revealed novel mechanism of the conformational transformation of the classical Watson-Crick G·C(WC) nucleobase pair into the reverse wobble Watson-Crick $\mathrm{G} \cdot \mathrm{C}\left(\mathrm{rw}_{\mathrm{WC}}\right)$ 
Table 1 Conformational transformations via the rotations of the $G$ and $C$ bases in the canonical and rare tautomeric forms around the intermolecular H-bonds in the Watson-Crick (WC), reverse Watson-Crick ( $r W C$ ), wobble Watson-Crick (wwc), reverse wobble Watson-Crick (rwwc), Hoogsteen $(\mathrm{H})$, reverse Hoogsteen $(\mathrm{rH})$, wobble Hoogsteen $\left(\mathrm{w}_{H}\right)$ and reverse wobble Hoogsteen $\left(r \mathrm{w}_{\mathrm{H}}\right) \mathrm{G} \cdot \mathrm{C}$ nucleobase pairs, obtained at the $\mathrm{MP} 2 / 6-311++\mathrm{G}(2 \mathrm{df}, \mathrm{pd}) / / \mathrm{B} 3 \mathrm{LYP} / 6-311++\mathrm{G}(\mathrm{d}, \mathrm{p})$ level of theory $\left(\Delta G\right.$ - relative Gibbs free and $\Delta E-$ relative electronic energies (in $\left.\mathrm{kcal}^{\mathrm{mol}}{ }^{-1}\right)$ in vacuum) under normal conditions $(T=298.15 \mathrm{~K})$. Intermolecular $\mathrm{AH} \cdots \mathrm{B} H$-bonds and $\mathrm{A} \cdots \mathrm{B}$ attractive van der Waals contacts are designated by the dotted lines, their lengths $H \cdots B$ and $A \cdots B$, respectively, are presented in Angstroms

Watson-Crick (WC), reverse Watson-Crick (rWC), wobble Watson-Crick $\left(\mathbf{w}_{\mathrm{WC}}\right)$ and reverse wobble Watson-Crick (rw (wC) configurations $\mathbf{G} \cdot \mathbf{C}(\mathbf{W C}) \leftrightarrow \mathbf{G} \cdot \mathbf{C}\left(\mathbf{r w}_{\mathrm{WC}}\right)\{$ rotation around the middle $\mathbf{N} 1 \mathrm{H} \cdots \mathbf{N} 3 \mathbf{H}-\mathrm{bond}\}$
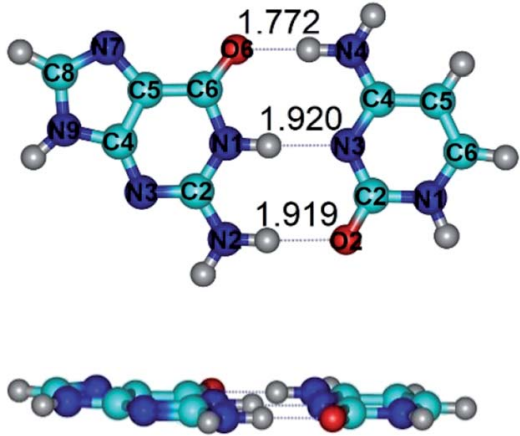

$$
\begin{gathered}
\mathrm{G} \cdot \mathrm{C}(\mathrm{WC}) \\
(\Delta G=0.00 / \Delta E=0.00 / \\
\mu=6.03)
\end{gathered}
$$
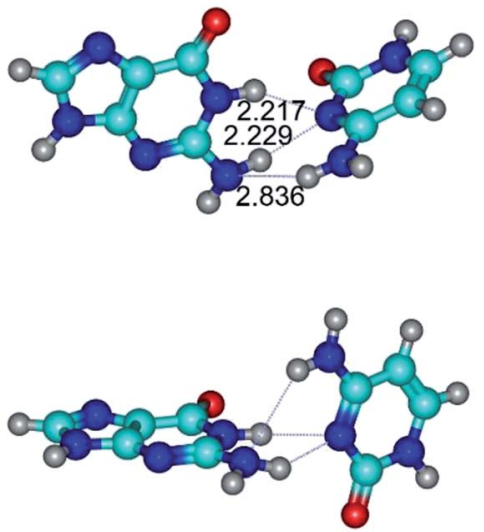

$$
\begin{gathered}
\mathrm{TS}_{\mathrm{G}} \cdot \mathrm{C}(\mathrm{WC}) \leftrightarrow \mathrm{G} \cdot \mathrm{C}\left(\mathrm{rw}_{\mathrm{Wc}}\right) \\
\left(\nu_{\mathrm{i}}=24.2 i \mathrm{~cm}^{-1}\right) \\
(\Delta G=12.63 / \Delta E=13.30 / \\
\mu=7.94)
\end{gathered}
$$

$\mathrm{G}^{+} \cdot \mathrm{C}^{-}(\mathrm{rWC}) \leftrightarrow \mathrm{G} \cdot \mathrm{C}(\mathrm{WC})\{$ rotation around the middle $\mathbf{N} 1 \mathrm{H} \cdots \mathrm{N} 3 \mathrm{H}-$ bond $\}$
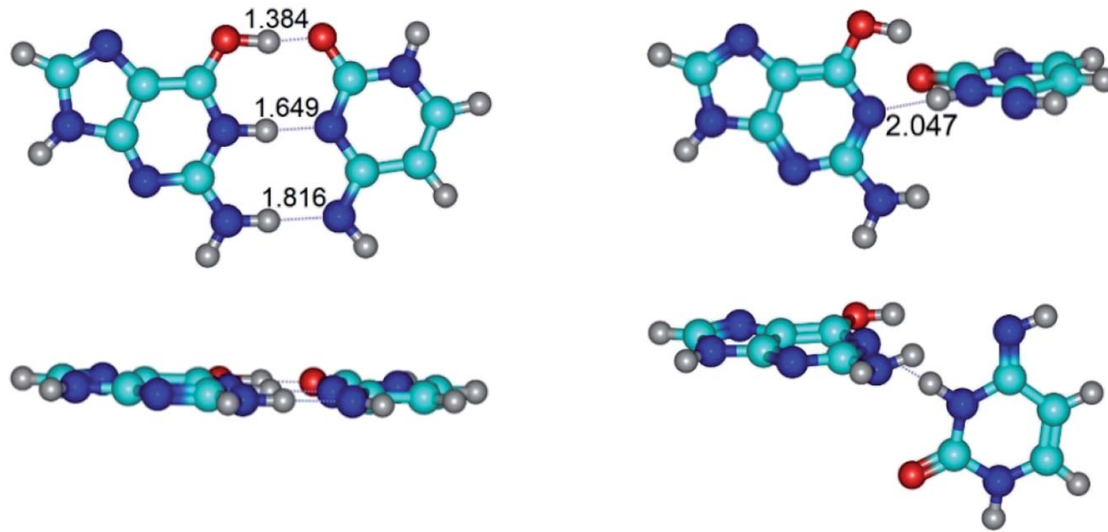

$$
\begin{gathered}
\mathrm{G}^{+} \cdot \mathrm{C}^{-}(\mathrm{rWC}) \\
(\Delta G=0.00 / \Delta E=0.00 / \\
\mu=3.20)
\end{gathered}
$$

$$
\begin{gathered}
\mathrm{TS}_{\mathrm{G}^{+} \cdot \mathrm{C}^{-}(\mathrm{rWC}) \leftrightarrow \mathrm{G} \cdot \mathrm{C}(\mathrm{WC})}\left(\nu_{\mathrm{i}}=37.2 i \mathrm{~cm}^{-1}\right) \\
(\Delta G=6.93 / \Delta E=7.06 / \\
\mu=4.12)
\end{gathered}
$$

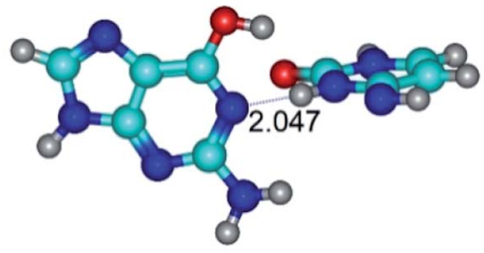

$\mathbf{G}^{*} \cdot \mathbf{C}^{*}(\mathrm{WC}) \leftrightarrow \mathrm{G}^{*} \cdot \mathrm{C}^{*}(\mathbf{r W C})\{$ rotation around the middle N3H $\cdots \mathbf{N} 1 \mathrm{H}-$ bond $\}$
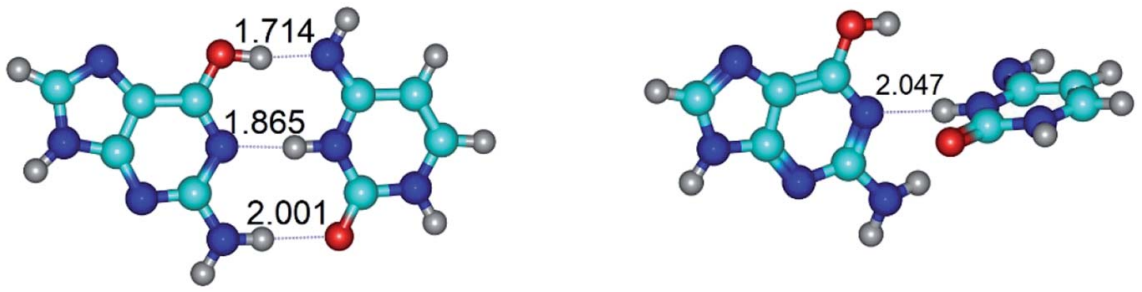
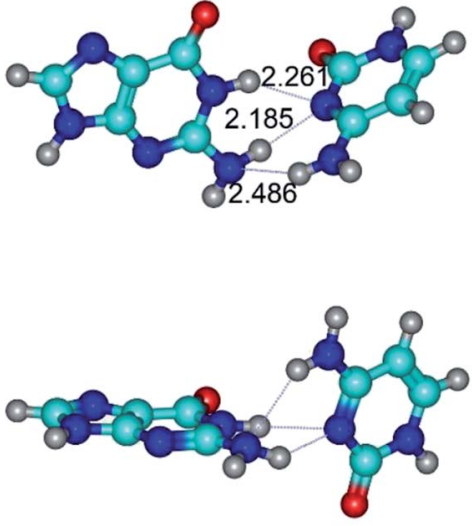

$\mathrm{G} \cdot \mathrm{C}\left(\mathrm{rw}_{\mathrm{WC}}\right)$

$(\Delta G=11.53 / \Delta E=13.09 / \mu=8.57)$
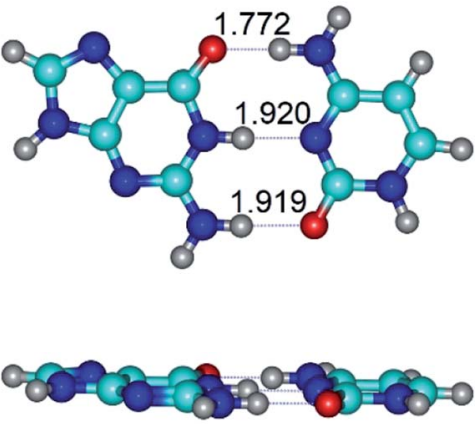

$\mathrm{G} \cdot \mathrm{C}(\mathrm{WC})$

$(\Delta G=-14.55 / \Delta E=-15.33 / \mu=6.03)$

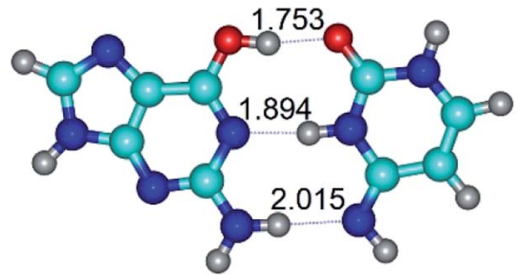




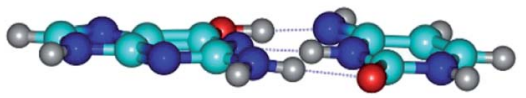

$\mathrm{G}^{*} \cdot \mathrm{C}^{*}(\mathrm{WC})$

$(\Delta G=0.00 / \Delta E=0.00 /$ $\mu=6.09)$

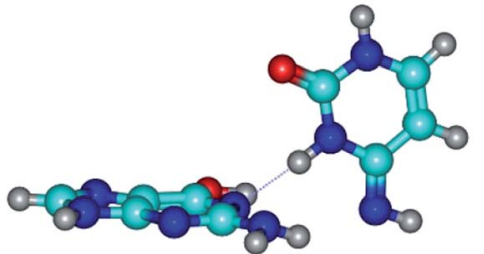

$\mathrm{TS}_{\mathrm{G}^{*} \cdot \mathrm{C}^{*}(\mathrm{WC}) \leftrightarrow \mathrm{G}^{*} \cdot \mathrm{C}^{*}(\mathrm{rWC})}$ $\left(\nu_{\mathrm{i}}=37.2 i \mathrm{~cm}^{-1}\right)$

$(\Delta G=13.11 / \Delta E=14.53 /$ $\mu=4.12$ )

$\mathbf{G}^{*} \cdot \mathbf{C}^{*}(\mathrm{WC}) \leftrightarrow \mathbf{G}^{*} \cdot \mathrm{C}^{*}\left(\mathrm{rw}_{\mathrm{WC} / \mathrm{H}}\right)$ rotation around the upper $\mathbf{O 6 H} \cdots \mathbf{N} 4 \mathrm{H}-$ bond $\}$
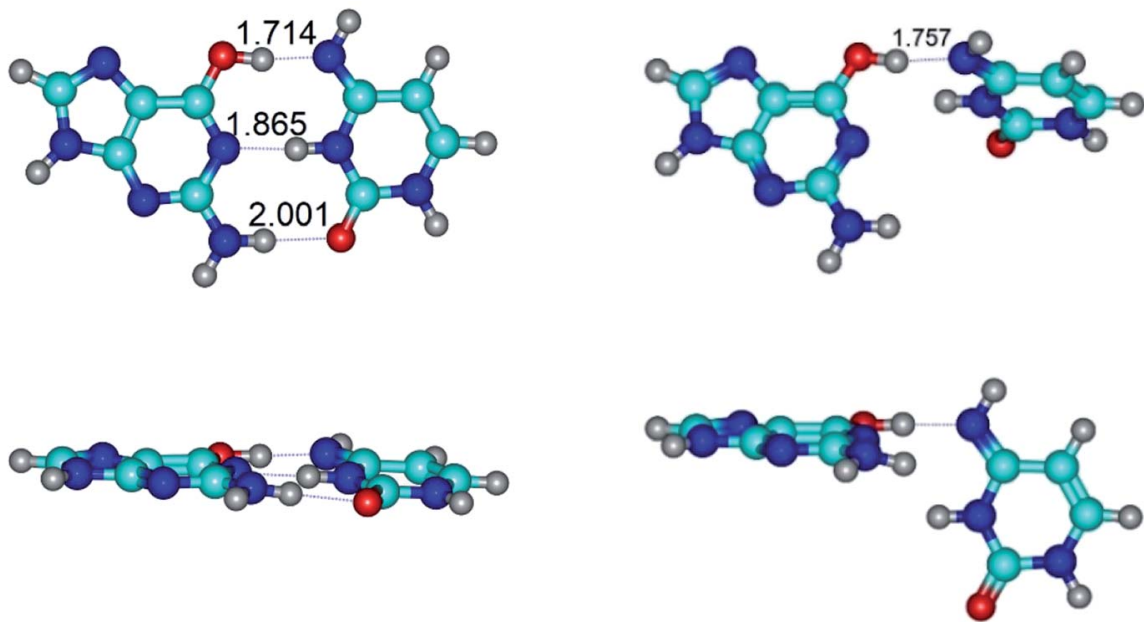

$$
\begin{gathered}
\mathrm{G}^{*} \cdot \mathrm{C}^{*}(\mathrm{WC}) \\
(\Delta G=0.00 / \Delta E=0.00 / \\
\mu=6.09)
\end{gathered}
$$

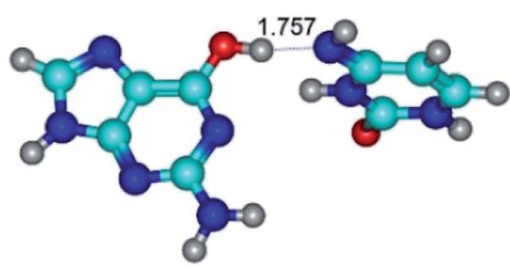

$$
\begin{gathered}
\mathrm{TS}_{\mathrm{G}^{*} \cdot \mathrm{C}^{*}(\mathrm{WC}) \leftrightarrow} \mathrm{G}^{*} \cdot \mathrm{C}^{*}\left(\mathrm{rw}_{\mathrm{WC} / \mathrm{H}}\right) \\
\left(\nu_{\mathrm{i}}=17.7 i \mathrm{~cm}^{-1}\right) \\
(\Delta G=10.05 / \Delta E=11.17 / \\
\mu=6.47)
\end{gathered}
$$

$\mathbf{G}^{*} \cdot \mathbf{C}^{*}(\mathbf{W C}) \leftrightarrow \mathbf{G}^{*} \cdot \mathbf{C}^{*}\left(\mathbf{r w}_{\mathbf{W C}}\right)\left\{\right.$ rotation around the lower $\mathbf{N} 2 \mathbf{H}^{\cdots} \mathbf{O} 2 \mathrm{H}-\mathrm{bond}$ \}
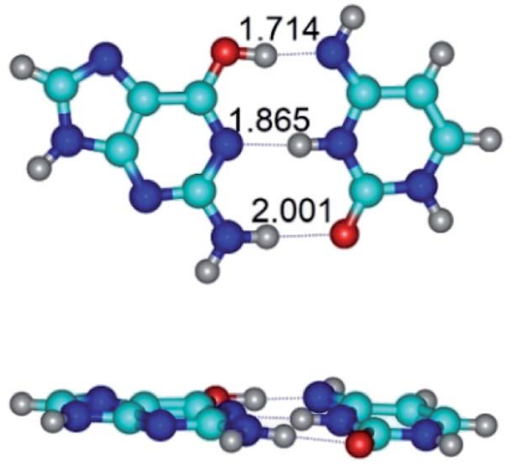

$\mathrm{G}^{*} \cdot \mathrm{C}^{*}(\mathrm{WC})$

$(\Delta G=0.00 / \Delta E=0.00 /$

$$
\mu=6.09)
$$
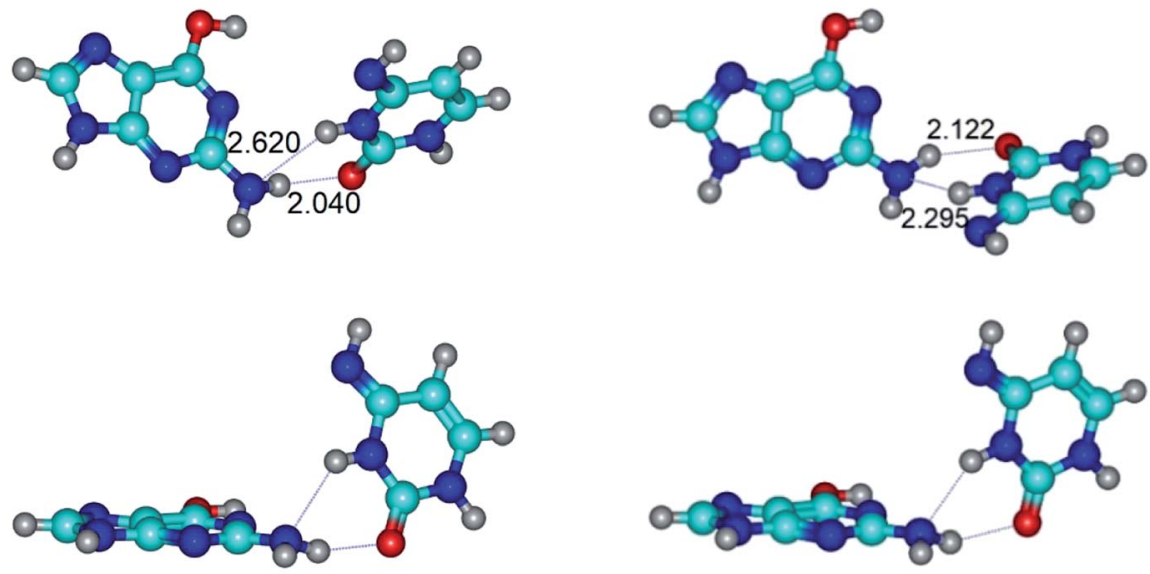

$$
\begin{gathered}
\mathrm{TS}_{\mathrm{G}^{*} \cdot \mathrm{C}^{*}(\mathrm{WC})} \leftrightarrow \mathrm{G}^{*} \cdot \mathrm{C}^{*}\left(\mathrm{rw}_{\mathrm{wc}}\right) \\
\left(\nu_{\mathrm{i}}=30.3 i \mathrm{~cm}^{-1}\right) \\
(\Delta G=12.98 / \Delta E=14.53 / \\
\mu=5.21)
\end{gathered}
$$

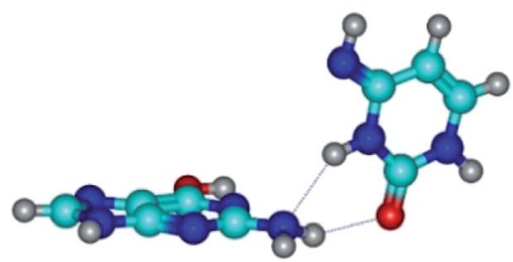

$\mathrm{G}^{*} \cdot \mathrm{C}^{*}\left(\mathrm{rw}_{\mathrm{WC}}\right)$

$(\Delta G=11.95 / \Delta E=14.30 / \mu=5.12)$

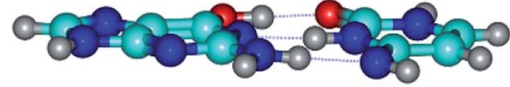

$\mathrm{G}^{*} \cdot \mathrm{C}^{*}(\mathrm{rWC})$

$(\Delta G=2.03 / \Delta E=2.07 / \mu=6.54)$
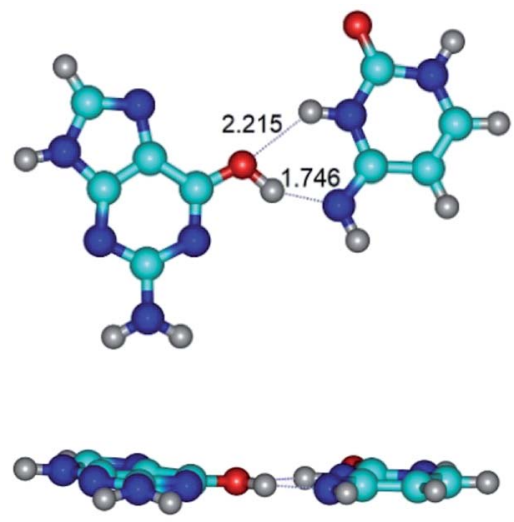

$$
\begin{gathered}
\mathrm{G}^{*} \cdot \mathrm{C}^{*}\left(\mathrm{rw}_{\mathrm{WC} / \mathrm{H}}\right) \\
(\Delta G=7.63 / \Delta E=10.21 / \mu=5.13)
\end{gathered}
$$


Table 1 (Contd.)

$\mathbf{G}^{*} \cdot \mathbf{C}^{*}(\mathbf{r W C}) \leftrightarrow \mathbf{G}^{*} \cdot \mathbf{C}^{*}\left(\mathbf{w}_{\mathrm{WC} / \mathrm{H}}\right)$ \{rotation around the upper $06 \mathrm{H} \cdots \mathbf{O} 2 \mathrm{H}-$-bond
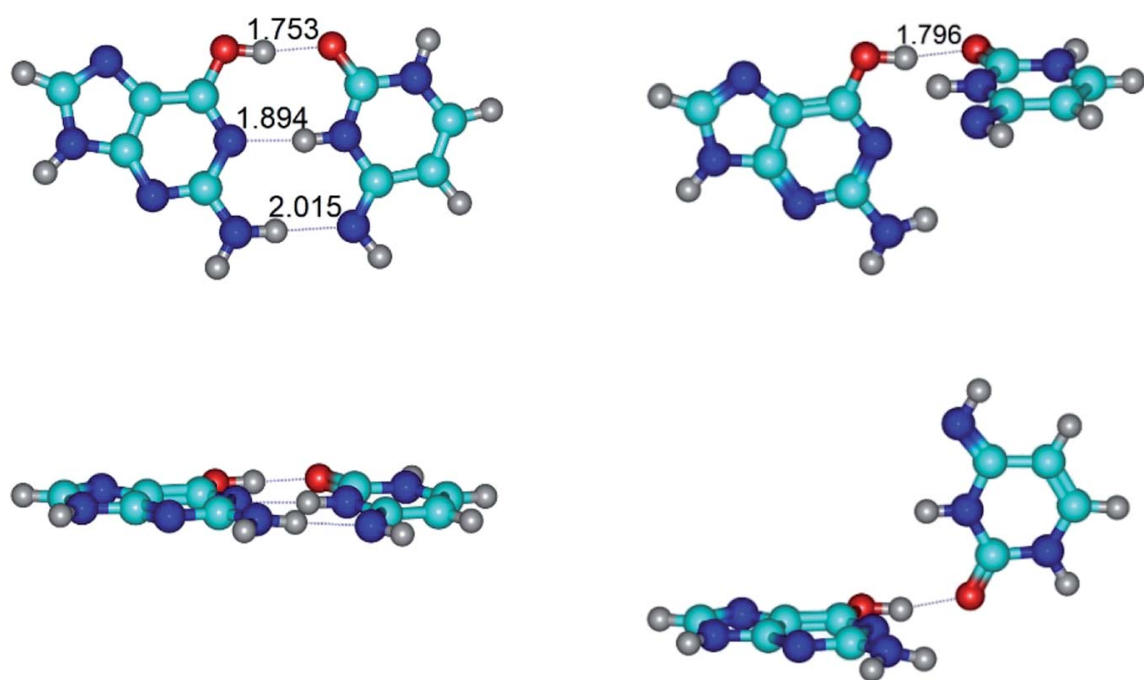

$$
\begin{gathered}
\mathrm{G}^{*} \cdot \mathrm{C}^{*}(\mathrm{rWC}) \\
(\Delta G=0.00 / \Delta E=0.00 / \\
\mu=6.54)
\end{gathered}
$$

$$
\begin{gathered}
\mathrm{TS}_{\mathrm{G}^{*} \cdot \mathrm{C}^{*}(\mathrm{rWC}) \leftrightarrow \mathrm{G}^{*} \cdot \mathrm{C}^{*}\left(\mathrm{w}_{\mathrm{WC} / \mathrm{H}}\right)} \\
\left(\nu_{\mathrm{i}}=11.8 i \mathrm{~cm}^{-1}\right) \\
(\Delta G=10.29 / \Delta E=11.67 / \\
\mu=6.09)
\end{gathered}
$$

$\mathbf{G}^{*} \cdot \mathbf{C}^{*}(\mathbf{r W C}) \leftrightarrow \mathbf{G}^{*} \cdot \mathbf{C}^{*}\left(\mathbf{w}_{\mathbf{W C}}\right)\left\{\right.$ rotation around the lower $\left.\mathbf{N} 2 \mathbf{H}^{\cdots} \mathbf{N} 4 \mathrm{H}-\mathrm{bond}\right\}$
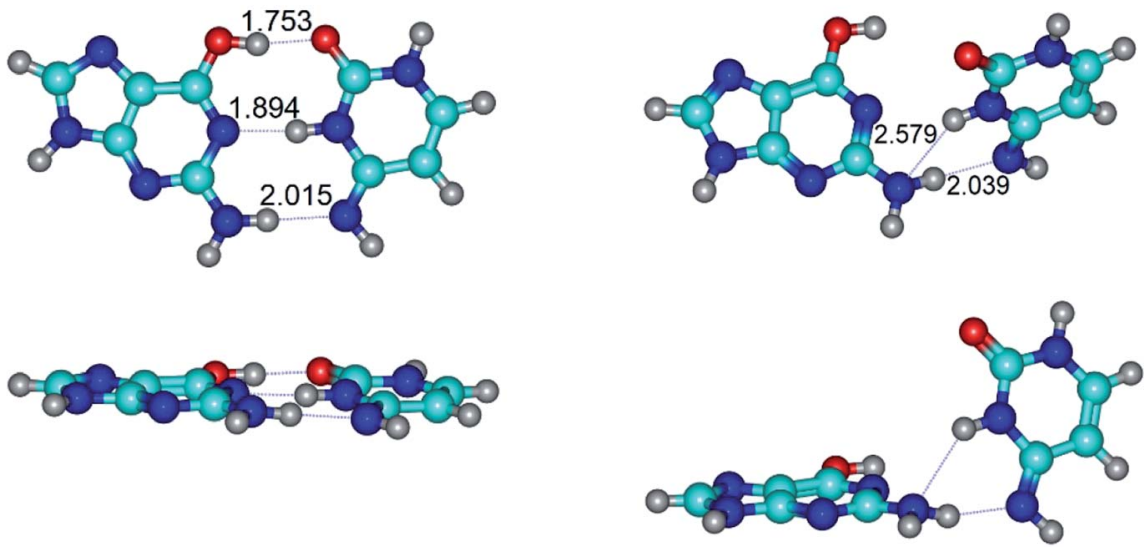

$$
\begin{gathered}
\mathrm{G}^{*} \cdot \mathrm{C}^{*}(\mathrm{rWC}) \\
(\Delta G=0.00 / \Delta E=0.00 / \\
\mu=6.54)
\end{gathered}
$$

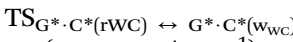

$$
\begin{aligned}
& \left(\nu_{\mathrm{i}}=33.3 i \mathrm{~cm}^{-1}\right) \\
& (\Delta G=9.95 / \Delta E=11.28 / \\
& \mu=5.86)
\end{aligned}
$$

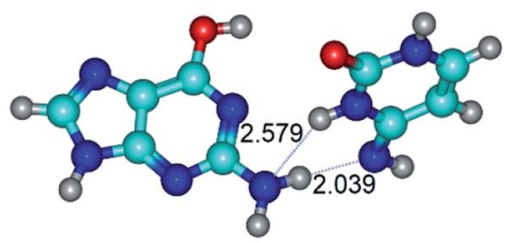

$\mathbf{G} \cdot \mathbf{C}^{*}{ }_{\mathrm{O} 2}(\mathrm{rWC}) \leftrightarrow \mathrm{G} \cdot \mathrm{C}^{*}{ }_{\mathrm{O} 2}\left(\mathbf{w}_{\mathrm{WC}}\right)$ \{rotation around the middle $\left.\mathbf{N} 1 \mathrm{H} \cdots \mathrm{N} 3 \mathrm{H}-\mathrm{bond}\right\}$
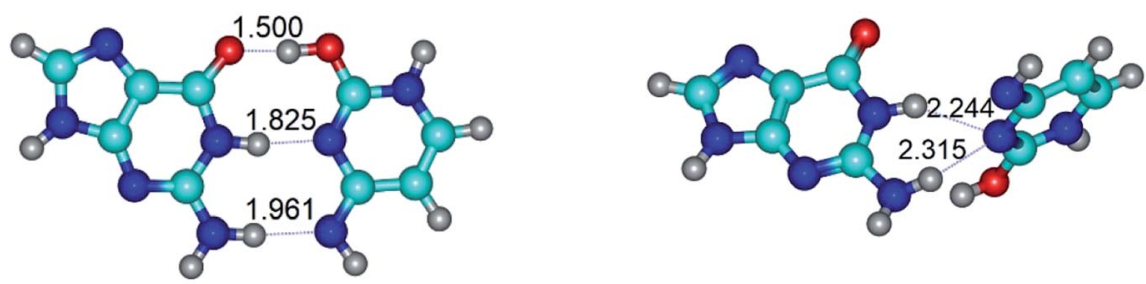
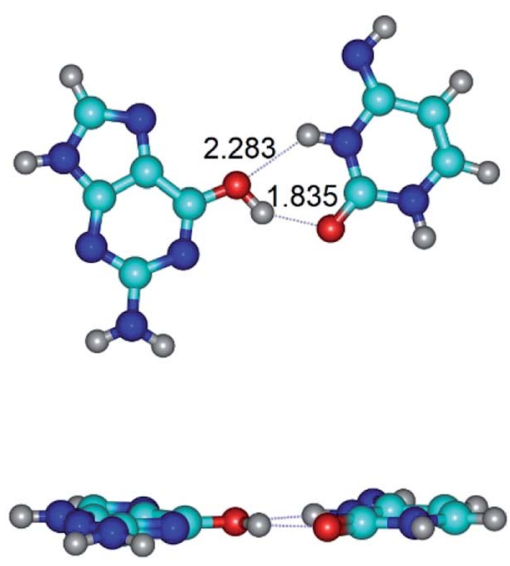

$$
\begin{gathered}
\mathrm{G}^{*} \cdot \mathrm{C}^{*}\left(\mathrm{w}_{\mathrm{WC} / \mathrm{H}}\right) \\
(\Delta G=8.40 / \Delta E=11.16 / \mu=4.00)
\end{gathered}
$$
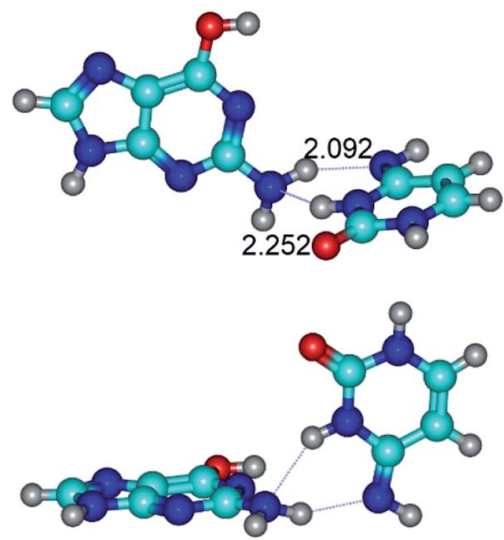

$\mathrm{G}^{*} \cdot \mathrm{C}^{*}\left(\mathrm{w}_{\mathrm{WC}}\right)$

$(\Delta G=8.76 / \Delta E=11.00 / \mu=5.48)$

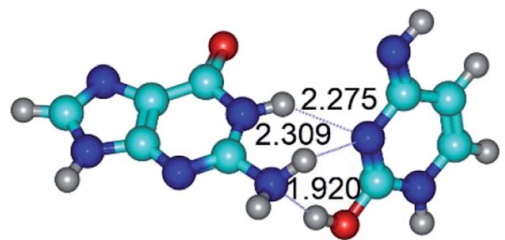




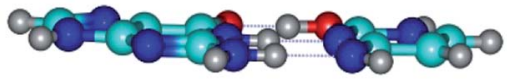

$$
\begin{gathered}
\mathrm{G} \cdot \mathrm{C}^{*}{ }_{\mathrm{O} 2}(\mathrm{rWC}) \\
(\Delta G=0.00 / \Delta E=0.00 / \\
\mu=5.43)
\end{gathered}
$$

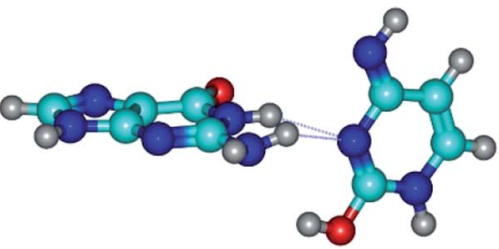

$$
\begin{gathered}
\mathrm{TS}_{\mathrm{G} \cdot \mathrm{C}^{*} \mathrm{O} 2(\mathrm{rWC}) \leftrightarrow \mathrm{G} \cdot \mathrm{C}^{*} \mathrm{O} 2\left(\mathrm{w}_{\mathrm{wc}}\right)}, \\
\left(\nu_{\mathrm{i}}=43.4 i \mathrm{~cm}^{-1}\right) \\
(\Delta G=19.04 / \Delta E=19.92 / \\
\mu=7.45)
\end{gathered}
$$

$\mathbf{G}^{*} \cdot \mathbf{C}^{*}\left(\mathrm{rw}_{\mathrm{WC} / \mathrm{H}}\right) \leftrightarrow \mathbf{G}^{*} \cdot \mathbf{C}^{*}\left(\mathbf{w}_{\mathrm{WC} / \mathrm{H}}\right)$ \{rotation around the lower $\mathbf{N} 3 \mathrm{H}^{\cdots} \mathrm{O}$. H-bond
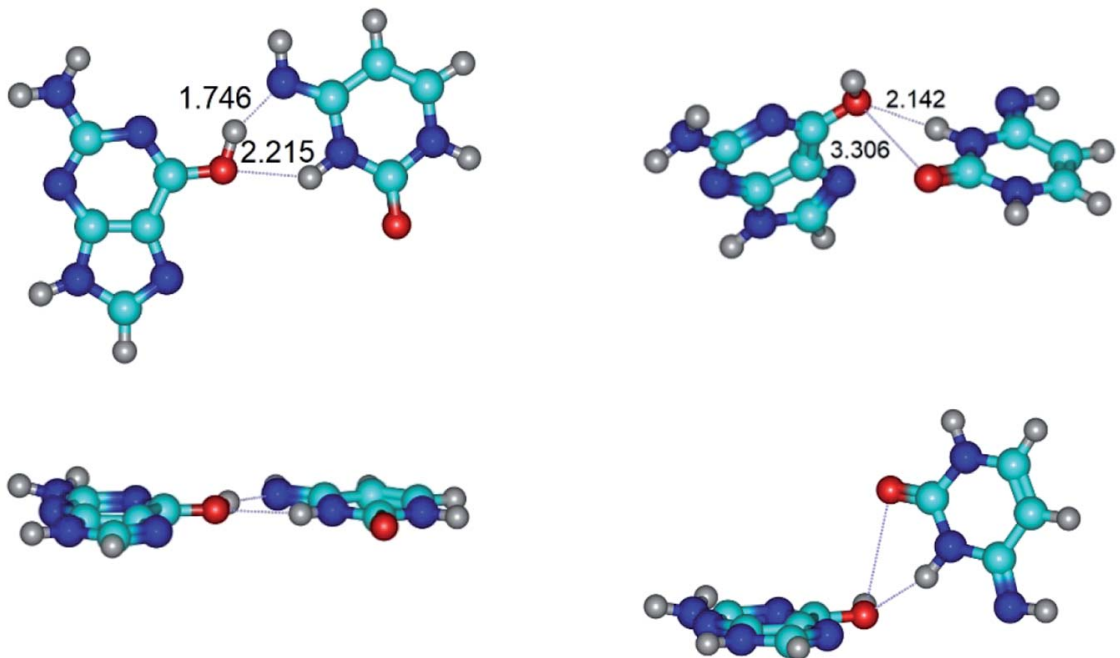

$$
\begin{gathered}
\mathrm{G}^{*} \cdot \mathrm{C} *\left(\mathrm{rw}_{\mathrm{WC} / \mathrm{H}}\right) \\
(\Delta G=0.00 / \Delta E=0.00 / \\
\mu=5.13)
\end{gathered}
$$

$\mathrm{TS}_{\mathrm{G}} * \mathrm{C}^{*}\left(\mathrm{rw}_{\mathrm{wC} / \mathrm{H}}\right) \leftrightarrow \mathrm{G}^{*} \cdot \mathrm{C}^{*}\left(\mathrm{w}_{\mathrm{wC} / \mathrm{H}}\right)$,

$$
\left(\nu_{\mathrm{i}}=18.8 i \mathrm{~cm}^{-1}\right)
$$

$(\Delta G=7.49 / \Delta E=7.16 /$

$$
\mu=3.94)
$$

$\mathbf{G}^{* \mathrm{t}} \cdot \mathbf{C}^{*}\left(\mathrm{rw}_{\mathrm{WC} / \mathrm{H}}\right) \leftrightarrow \mathbf{G}^{* \mathrm{t}} \cdot \mathbf{C}^{*}\left(\mathbf{w}_{\mathrm{WC} / \mathrm{H}}\right)$ \{rotation around the upper N3H $\cdots \mathrm{O}$ H-bond
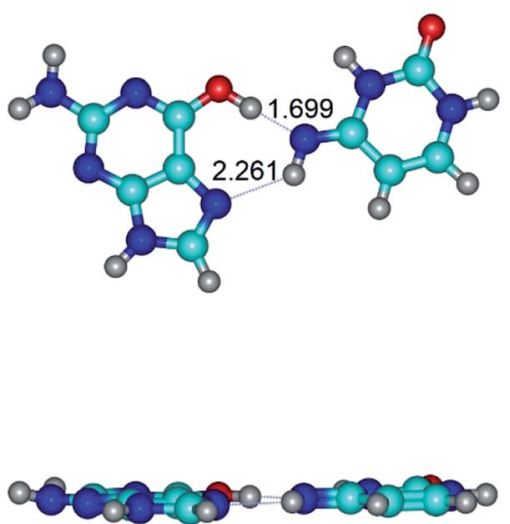

$$
\begin{gathered}
\mathrm{G}^{* \mathrm{t}} \cdot \mathrm{C}^{*}\left(\mathrm{rw}_{\mathrm{WC} / \mathrm{H}}\right) \\
(\Delta G=0.00 / \Delta E=0.00 / \\
\mu=5.14)
\end{gathered}
$$
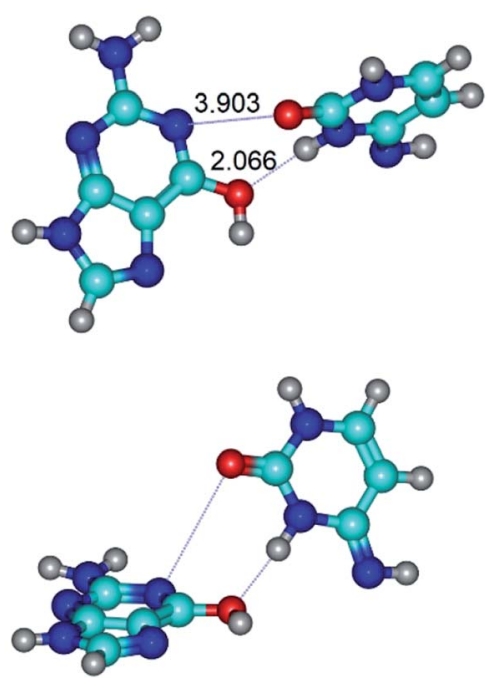

$$
\begin{gathered}
{ }_{\mathrm{H}}\left(\nu_{\mathrm{i}}=11.2 i \mathrm{~cm}^{-1}\right) \\
\Delta G=8.48 / \Delta E=8.35 / \\
\mu=3.30)
\end{gathered}
$$

$\mathrm{TS}_{\mathrm{G}} * \mathrm{t} \cdot \mathrm{C}^{*}\left(\mathrm{rw}_{\mathrm{WC} / \mathrm{H}}\right) \leftrightarrow \mathrm{G}^{*} \mathrm{t} \cdot \mathrm{C}^{*}\left(\mathrm{w}_{\mathrm{WC} /}\right.$

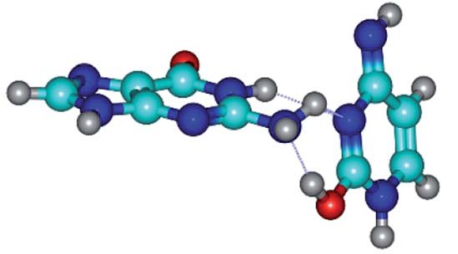

$\mathrm{G} \cdot \mathrm{C}^{*}{ }_{\mathrm{O} 2}\left(\mathrm{w}_{\mathrm{WC}}\right)$

$(\Delta G=16.74 / \Delta E=17.33 / \mu=7.91)$
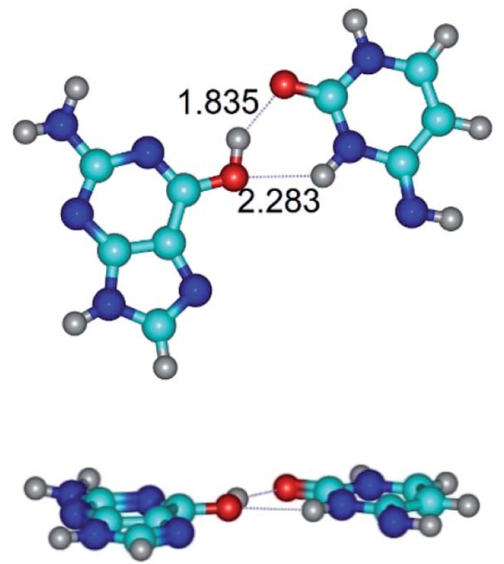

$\mathrm{G}^{*} \cdot \mathrm{C}^{*}\left(\mathrm{~W}_{\mathrm{WC} / \mathrm{H}}\right)$

$(\Delta G=2.80 / \Delta E=3.02 / \mu=4.00)$
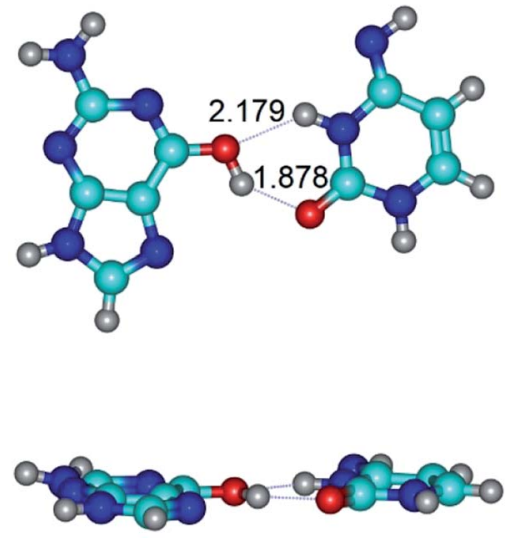

$\mathrm{G}^{* \mathrm{t}} \cdot \mathrm{C}^{*}\left(\mathrm{~W}_{\mathrm{WC} / \mathrm{H}}\right)$

$(\Delta G=4.37 / \Delta E=4.76 / \mu=4.12)$ 
$\mathbf{G}^{* \mathrm{t}} \cdot \mathbf{C}^{*}(\mathbf{r W C}) \leftrightarrow \mathbf{G}^{* \mathrm{t}} \cdot \mathbf{C}^{*}(\mathrm{WC})\{$ rotation around the middle N3H $\cdots \mathrm{N} 1 \mathrm{H}-$ bond
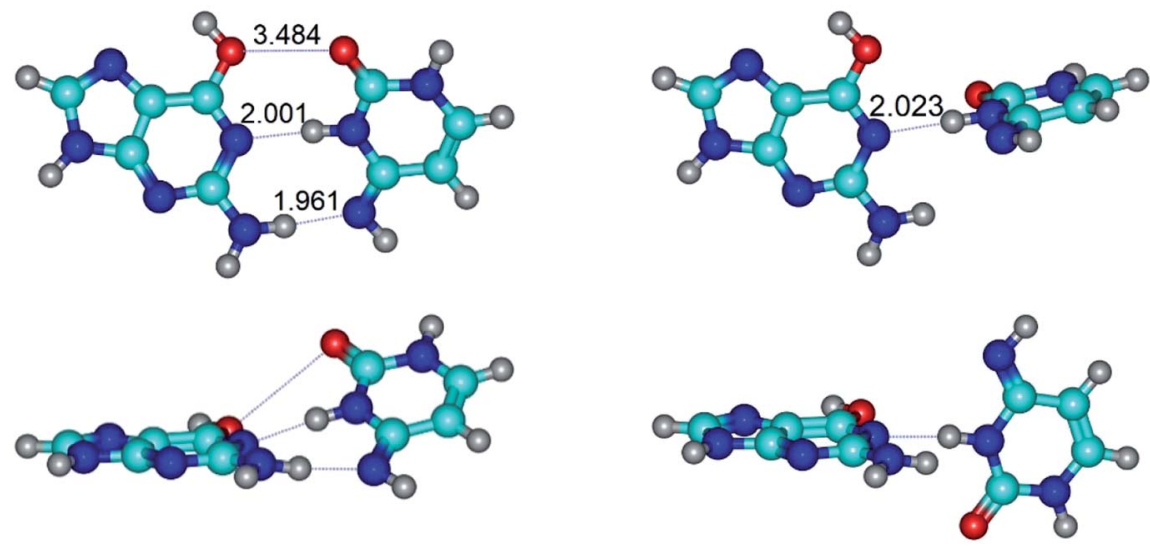

$$
\begin{gathered}
\mathrm{G}^{* \mathrm{t}} \cdot \mathrm{C}^{*}(\mathrm{rWC}) \\
(\Delta G=0.00 / \Delta E=0.00 / \\
\mu=2.54)
\end{gathered}
$$

$$
\begin{gathered}
\mathrm{TS}_{\mathrm{G}^{*} \mathrm{t} \cdot \mathrm{C}^{*}(\mathrm{rWC}) \leftrightarrow \mathrm{G}^{*} \cdot \mathrm{C}^{*}(\mathrm{WC})} \\
\left(\nu_{\mathrm{i}}=24.0 i \mathrm{~cm}^{-1}\right) \\
(\Delta G=5.23 / \Delta E=6.28 / \\
\mu=1.08)
\end{gathered}
$$

$\mathbf{G}^{* \mathrm{t}} \cdot \mathbf{C}^{*}(\mathbf{r W C}) \leftrightarrow \mathbf{G}^{* \mathrm{t}} \cdot \mathbf{C}^{*}\left(\mathbf{w}_{\mathrm{WC}}\right)\{$ rotation around the lower N2H $\cdots \mathbf{N} 4 \mathrm{H}-$ bond $\}$
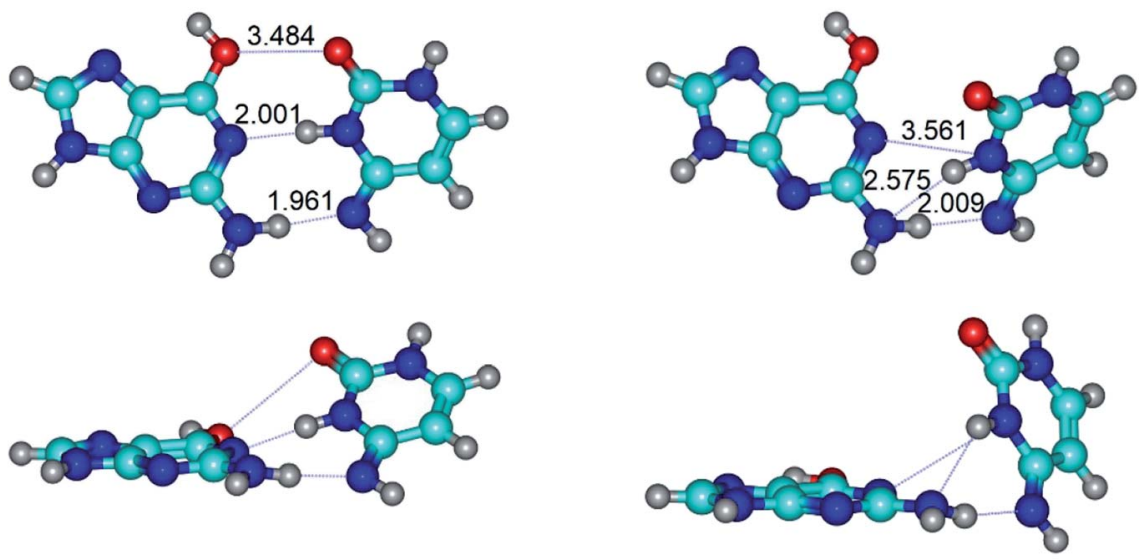

$$
\begin{gathered}
\mathrm{G}^{* \mathrm{t}} \cdot \mathrm{C}^{*}(\mathrm{rWC}) \\
(\Delta G=0.00 / \Delta E=0.00 / \\
\mu=2.54)
\end{gathered}
$$

$$
\begin{gathered}
\mathrm{TS}_{\mathrm{G}^{*} \mathrm{t} \cdot \mathrm{C}^{*}(\mathrm{rWC}) \leftrightarrow \mathrm{G}^{*} \mathrm{t} \cdot \mathrm{C}^{*}\left(\mathrm{w}_{\mathrm{WC}}\right)} \\
\left(\nu_{\mathrm{i}}=14.6 i \mathrm{~cm}^{-1}\right) \\
(\Delta G=4.13 / \Delta E=3.97 / \\
\mu=2.10)
\end{gathered}
$$

$\mathbf{G} \cdot \mathrm{C}^{* \mathrm{t}}{ }_{\mathrm{O} 2}(\mathrm{rWC}) \leftrightarrow \mathrm{G} \cdot \mathrm{C}^{* \mathrm{t}}{ }_{\mathrm{O} 2}\left(\mathbf{w}_{\mathrm{WC}}\right)$ \{rotation around the middle N1H $\left.\cdots \mathrm{N} 3 \mathrm{H}-\mathrm{bond}\right\}$
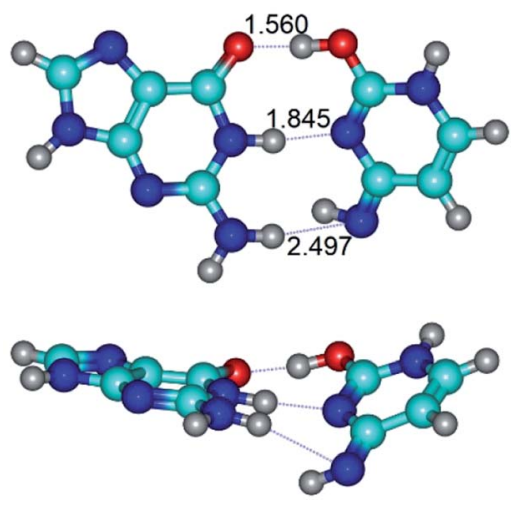
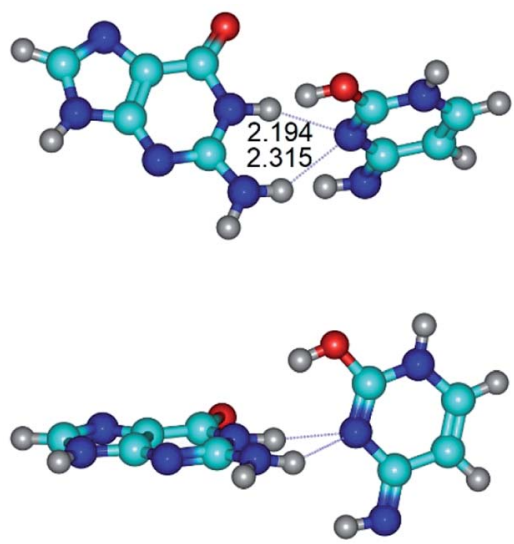
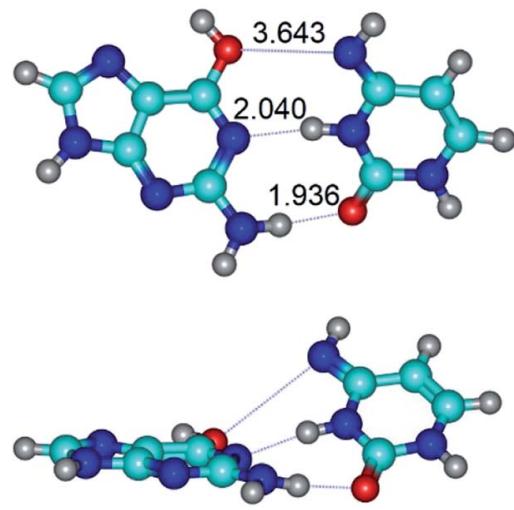

$$
\begin{gathered}
\mathrm{G}^{* \mathrm{t}} \cdot \mathrm{C}^{*}(\mathrm{WC}) \\
(\Delta G=1.34 / \Delta E=1.54 / \mu=1.91)
\end{gathered}
$$
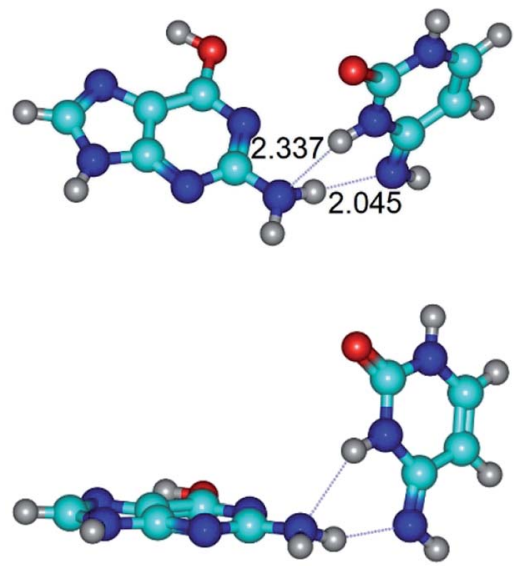

$$
(\Delta G=2.74 / \Delta E=4.24 / \mu=1.95)
$$
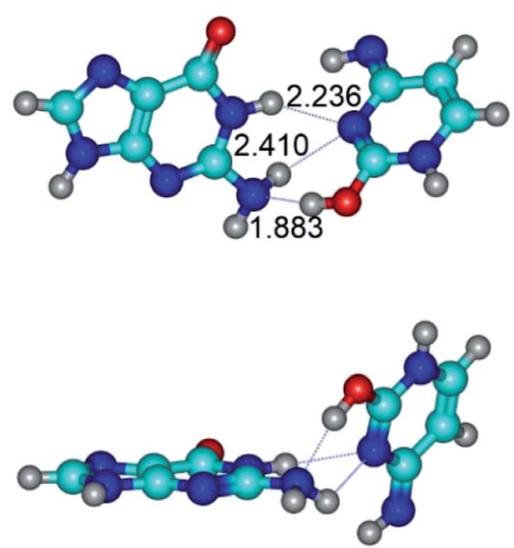
Table 1 (Contd.)

$$
\begin{array}{cc}
\mathrm{G} \cdot \mathrm{C}^{* \mathrm{t}}{ }_{\mathrm{O} 2}(\mathrm{rWC}) & \mathrm{TS}_{\mathrm{G}} \cdot \mathrm{C}^{*} \mathrm{tO} 2(\mathrm{rWC}) \leftrightarrow \mathrm{G}^{\mathrm{G}} \cdot \mathrm{C}^{*} \mathrm{tO}_{2}\left(\mathrm{w}_{\mathrm{WC}}\right) \\
(\Delta G=0.00 / \Delta E=0.00 / & \left(\nu_{\mathrm{i}}=36.4 i \mathrm{~cm}^{-1}\right) \\
\mu=3.32) & (\Delta G=12.28 / \Delta E=13.65 / \\
& \mu=7.40)
\end{array}
$$$$
\mathrm{G} \cdot \mathrm{C}^{* \mathrm{t}}{ }_{\mathrm{O} 2}\left(\mathrm{~W}_{\mathrm{WC}}\right)
$$$$
\Delta G=9.20 / \Delta E=9.84 / \mu=8.65)
$$

$\mathbf{G}^{* \mathrm{t}} \cdot \mathbf{C}^{* \mathrm{t}}(\mathrm{WC}) \leftrightarrow \mathbf{G}^{* \mathrm{t}} \cdot \mathbf{C}^{* \mathrm{t}}\left(\mathbf{r w}_{\mathrm{WC}}\right)_{\uparrow}\{$ rotation around the middle N3H $\cdots \mathbf{N} 1$ H-bond
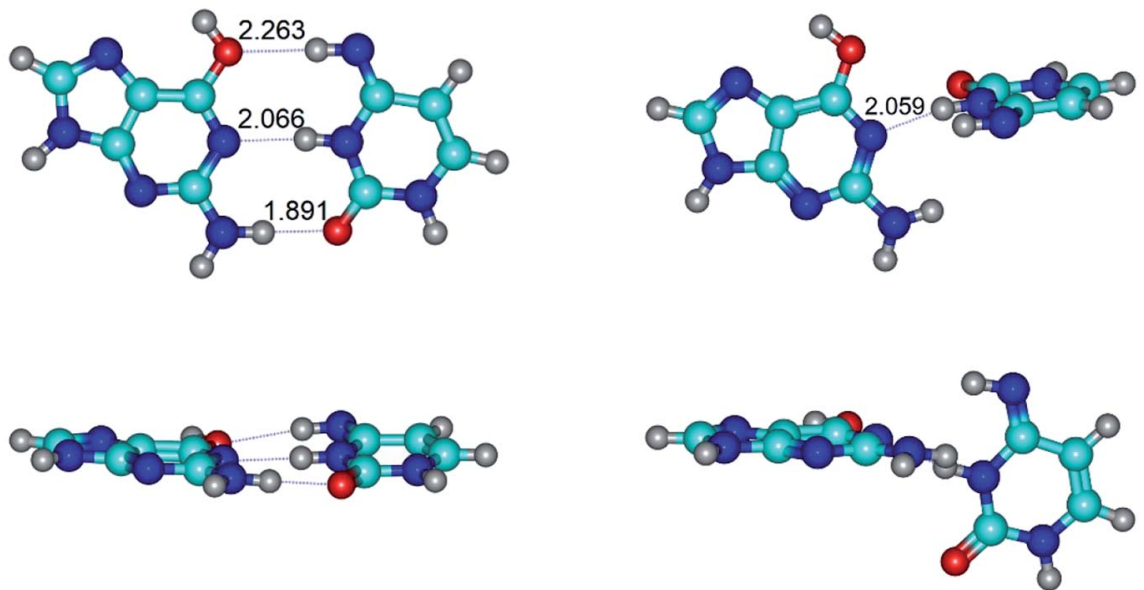

$$
\begin{gathered}
\mathrm{G}^{* \mathrm{t}} \cdot \mathrm{C}^{* \mathrm{t}}(\mathrm{WC}) \\
(\Delta G=0.00 / \Delta E=0.00 / \\
\mu=2.99)
\end{gathered}
$$

$$
\begin{gathered}
\mathrm{TS}_{\mathrm{G}^{*} \mathrm{t} \cdot \mathrm{C} * \mathrm{t}(\mathrm{WC})} \leftrightarrow \mathrm{G}^{*} \mathrm{t} \cdot \mathrm{C}^{*} \mathrm{t}\left(\mathrm{rw} \mathrm{wC}_{\mathrm{wC}}\right)_{\uparrow} \\
\left(\nu_{\mathrm{i}}=21.7 i \mathrm{~cm}^{-1}\right) \\
(\Delta G=5.05 / \Delta E=6.01 / \\
\mu=3.51)
\end{gathered}
$$

$\mathbf{G}^{* \mathrm{t}} \cdot \mathbf{C}^{* \mathrm{t}}(\mathrm{WC}) \leftrightarrow \mathbf{G}^{* \mathrm{t}} \cdot \mathbf{C}^{* \mathrm{t}}\left(\mathrm{rw}_{\mathrm{WC}}\right)$ \{rotation around the lower $\mathbf{N} 2 \mathrm{H} \cdots \mathbf{O} 2 \mathrm{H}-$-bond
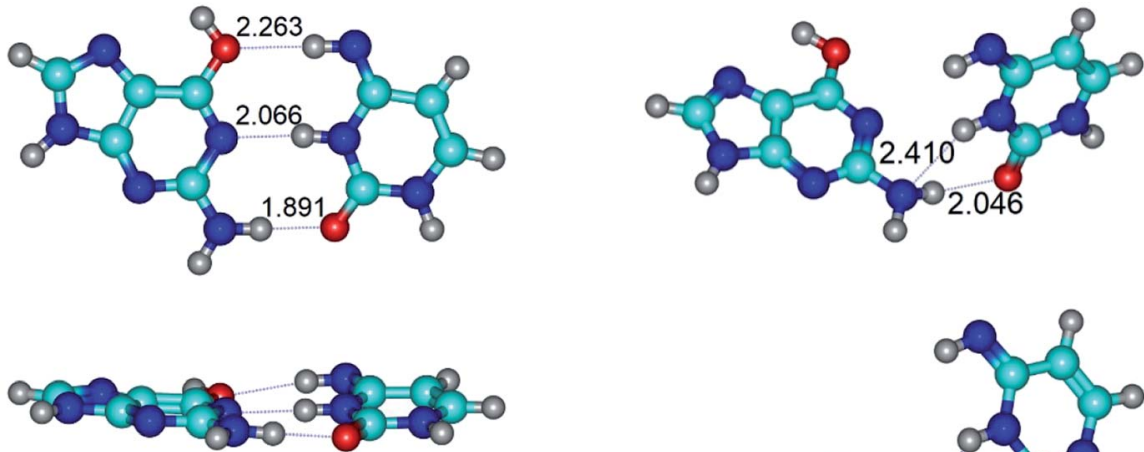

$$
\begin{gathered}
\mathrm{G}^{* \mathrm{t}} \cdot \mathrm{C}^{* \mathrm{t}}(\mathrm{WC}) \\
(\Delta G=0.00 / \Delta E=0.00 / \\
\mu=2.99)
\end{gathered}
$$

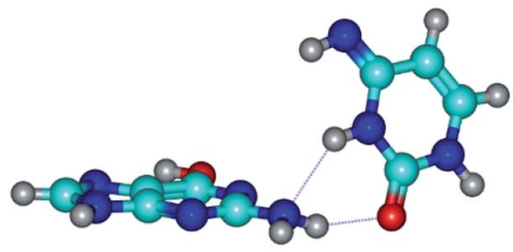

$$
\begin{gathered}
\mathrm{TS}_{\mathrm{G}^{*} \mathrm{t} \cdot \mathrm{C}^{*} \mathrm{t}(\mathrm{WC}) \leftrightarrow \mathrm{G}^{*} \cdot \mathrm{C}^{*}(\mathrm{rw}}\left(\mathrm{rw}_{\mathrm{wC}}\right) \\
\left(\nu_{\mathrm{i}}=27.3 i \mathrm{~cm}^{-1}\right) \\
(\Delta G=6.69 / \Delta E=6.91 / \\
\mu=1.92)
\end{gathered}
$$

$\mathbf{G} \cdot \mathbf{C}^{*}\left(\mathbf{w}_{\mathbf{W C}}\right)_{\uparrow} \leftrightarrow \mathbf{G} \cdot \mathbf{C}^{*}\left(\mathbf{r w}_{\mathbf{W C}}\right)$ rotation around the lower N1H $\cdots \mathbf{O} 2 \mathbf{H}-$ bond

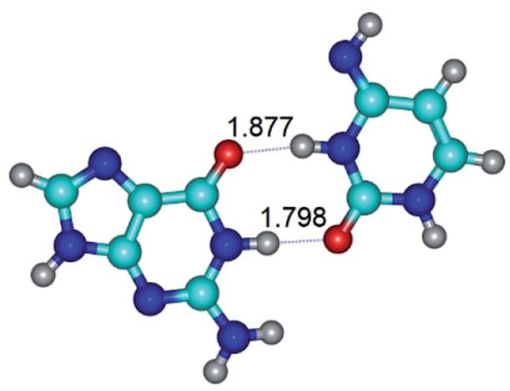

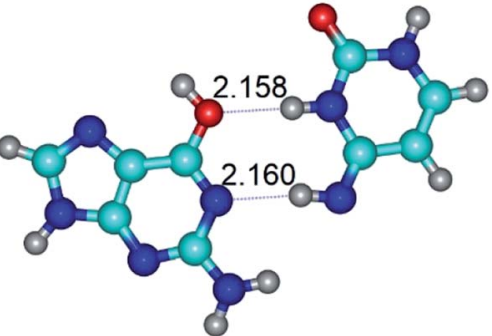

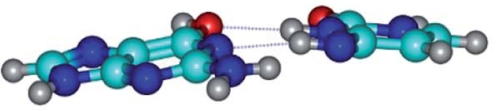

$$
\begin{gathered}
\mathrm{G}^{* \mathrm{t}} \cdot \mathrm{C}^{* \mathrm{t}}\left(\mathrm{rw}_{\mathrm{WC}}\right)_{\uparrow} \\
(\Delta G=3.69 / \Delta E=5.37 / \mu=3.00)
\end{gathered}
$$
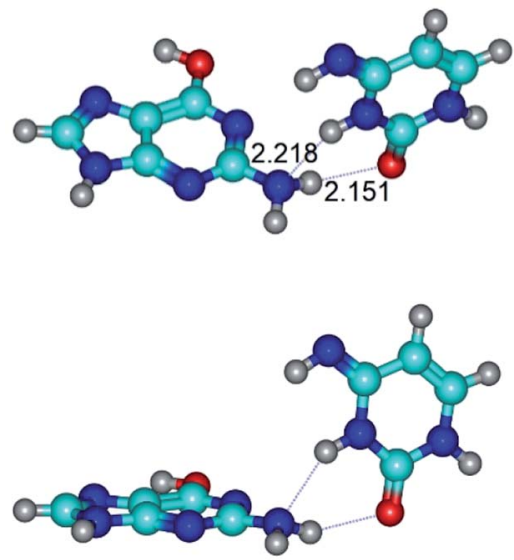

$\mathrm{G}^{* \mathrm{t}} \cdot \mathrm{C}^{* \mathrm{t}}\left(\mathrm{rw}_{\mathrm{WC}}\right)$

$(\Delta G=5.36 / \Delta E=7.03 / \mu=1.98)$

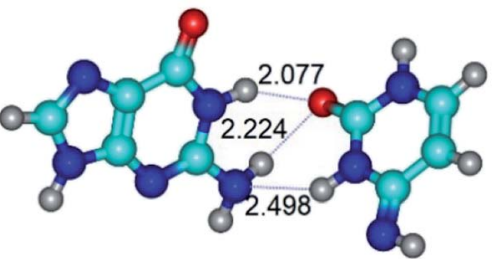



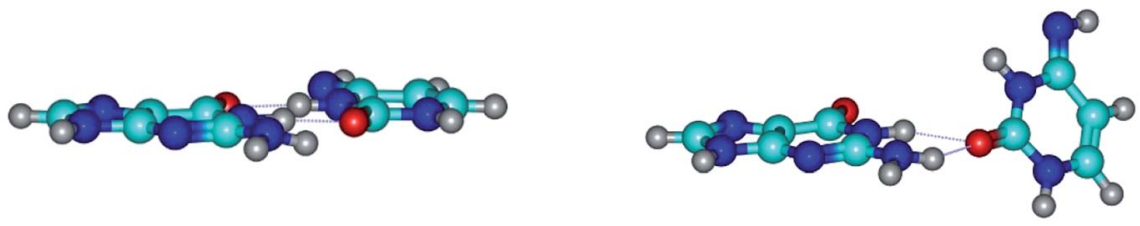

$$
\begin{gathered}
\mathrm{G} \cdot \mathrm{C}^{*}\left(\mathrm{~W}_{\mathrm{WC}}\right)_{\uparrow} \\
(\Delta G=0.00 / \Delta E=0.00 / \\
\mu=7.29)
\end{gathered}
$$

$$
\begin{gathered}
\mathrm{TS}_{\mathrm{G} \cdot \mathrm{C}^{*}\left(\mathrm{w}_{\mathrm{wC}}\right)_{\uparrow} \leftrightarrow \mathrm{G} \cdot \mathrm{C}^{*}(\mathrm{rwWC})}\left(\nu_{\mathrm{i}}=18.1 i \mathrm{~cm}^{-1}\right) \\
(\Delta G=4.97 / \Delta E=5.50 / \\
\mu=9.37)
\end{gathered}
$$

$\mathbf{G} \cdot \mathbf{C}^{*}\left(\mathbf{r w}_{\mathbf{W C}}\right) \leftrightarrow \mathbf{G} \cdot \mathbf{C}^{*}\left(\mathbf{w}_{\mathbf{W C}}\right)\{$ rotation around the lower $\mathbf{N} 3 \mathbf{H} \cdots \mathbf{N} 2 \mathrm{H}-$ bond
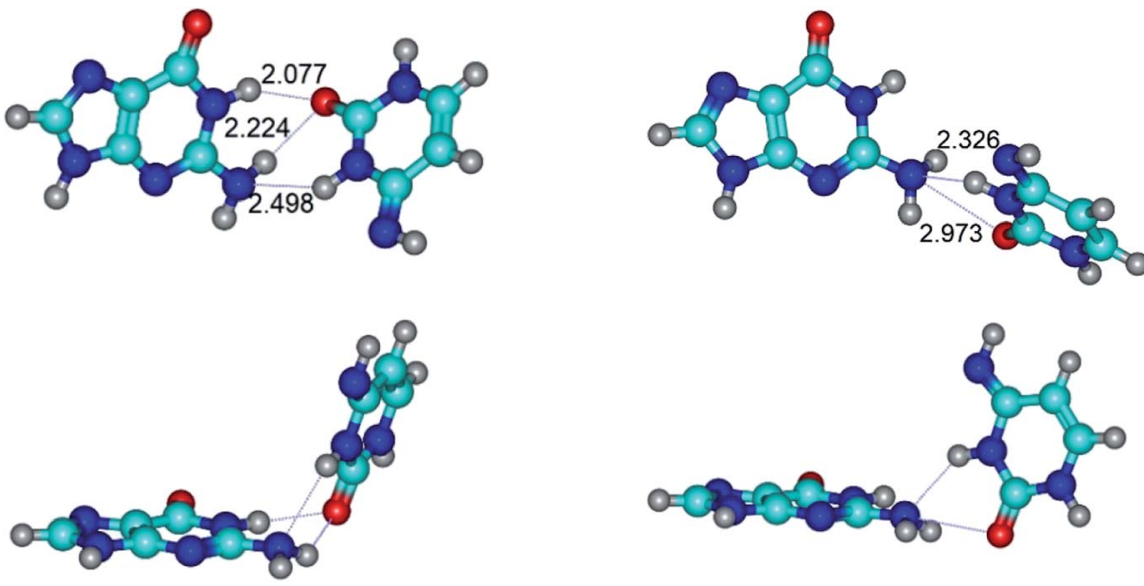

$$
\begin{gathered}
\mathrm{G} \cdot \mathrm{C}^{*}\left(\mathrm{rw}_{\mathrm{WC}}\right) \\
(\Delta G=0.00 / \Delta E=0.00 / \\
\mu=6.94)
\end{gathered}
$$

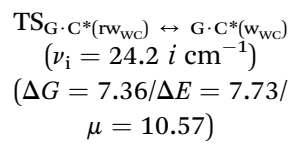

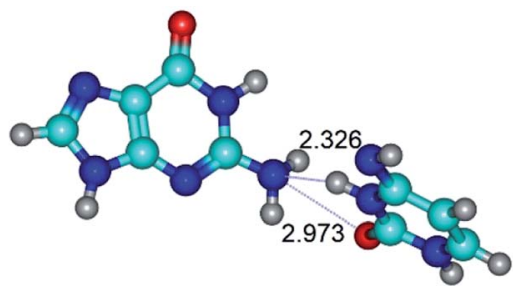

$\mathbf{G} \cdot \mathbf{C}^{*}\left(\mathbf{r w}_{\mathbf{W C}}\right)_{\uparrow} \leftrightarrow \mathbf{G} \cdot \mathbf{C}^{*}\left(\mathbf{w}_{\mathbf{W C}}\right)_{\uparrow}$ \{rotation around the upper N3H $\cdots$ O6 H-bond
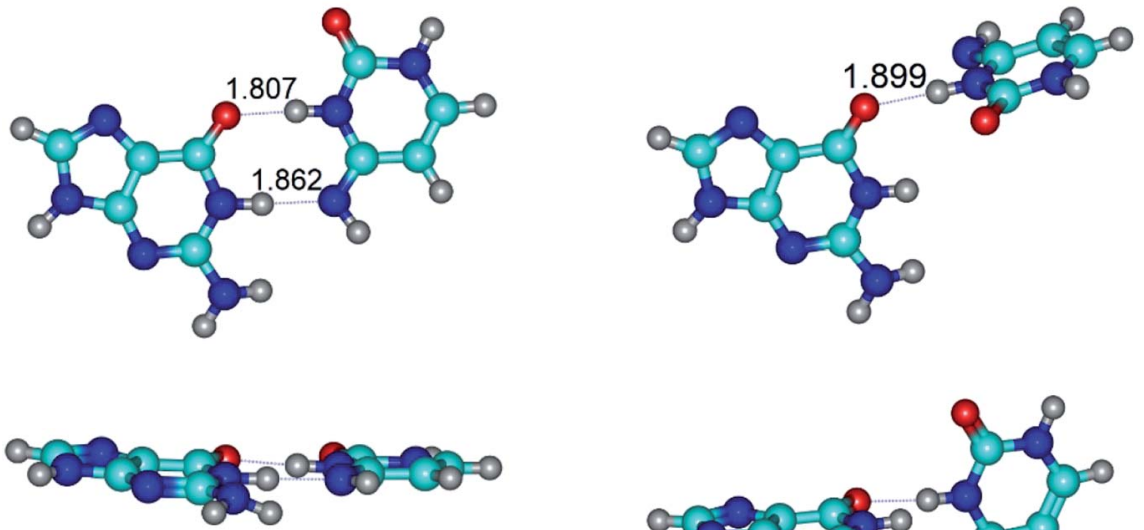

$\mathrm{G} \cdot \mathrm{C} *\left(\mathrm{rw}_{\mathrm{WC}}\right)_{\uparrow}$

$(\Delta G=0.00 / \Delta E=0.00 /$

$$
\mu=7.74)
$$

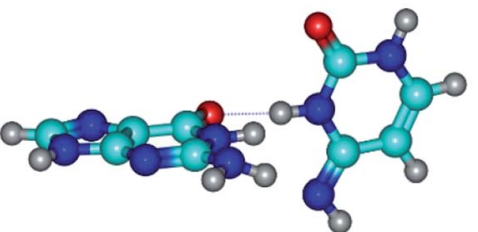

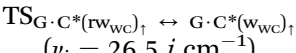

$$
\left(\nu_{\mathrm{i}}=26.5 i \mathrm{~cm}^{-1}\right)
$$

$(\Delta G=9.98 / \Delta E=11.47 /$

$$
\mu=6.10)
$$

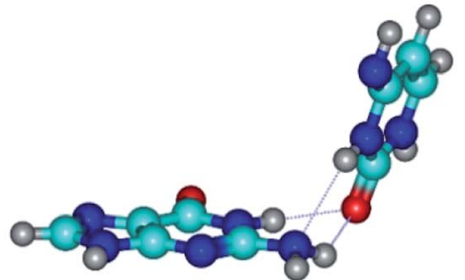

$\mathrm{G} \cdot \mathrm{C}^{*}\left(\mathrm{rw}_{\mathrm{WC}}\right)$

$(\Delta G=3.20 / \Delta E=3.88 / \mu=6.94)$
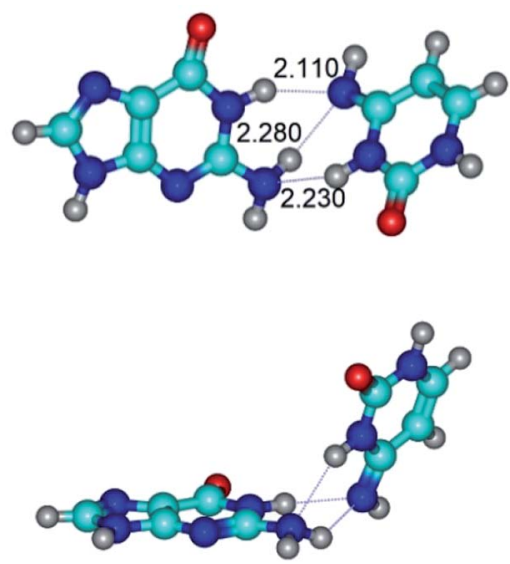

$$
\begin{gathered}
\mathrm{G} \cdot \mathrm{C} *\left(\mathrm{w}_{\mathrm{WC}}\right) \\
(\Delta G=1.83 / \Delta E=2.37 / \mu=7.36)
\end{gathered}
$$
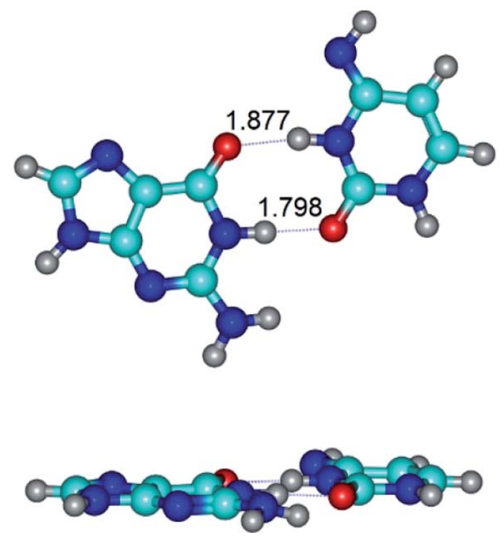

$\mathrm{G} \cdot \mathrm{C} *\left(\mathrm{~W}_{\mathrm{WC}}\right)_{\uparrow}$

$(\Delta G=1.38 / \Delta E=1.55 / \mu=7.29)$ 
Table 1 (Contd.)

$\mathbf{G} \cdot \mathbf{C}^{*}\left(\mathbf{r w}_{\mathrm{WC}}\right)_{\uparrow} \leftrightarrow \mathbf{G} \cdot \mathbf{C}^{*}\left(\mathbf{w}_{\mathrm{WC}}\right)$ \{rotation around the lower $\left.\mathbf{N} 1 \mathrm{H}^{\cdots} \mathrm{N} 4 \mathrm{H}-\mathrm{bond}\right\}$
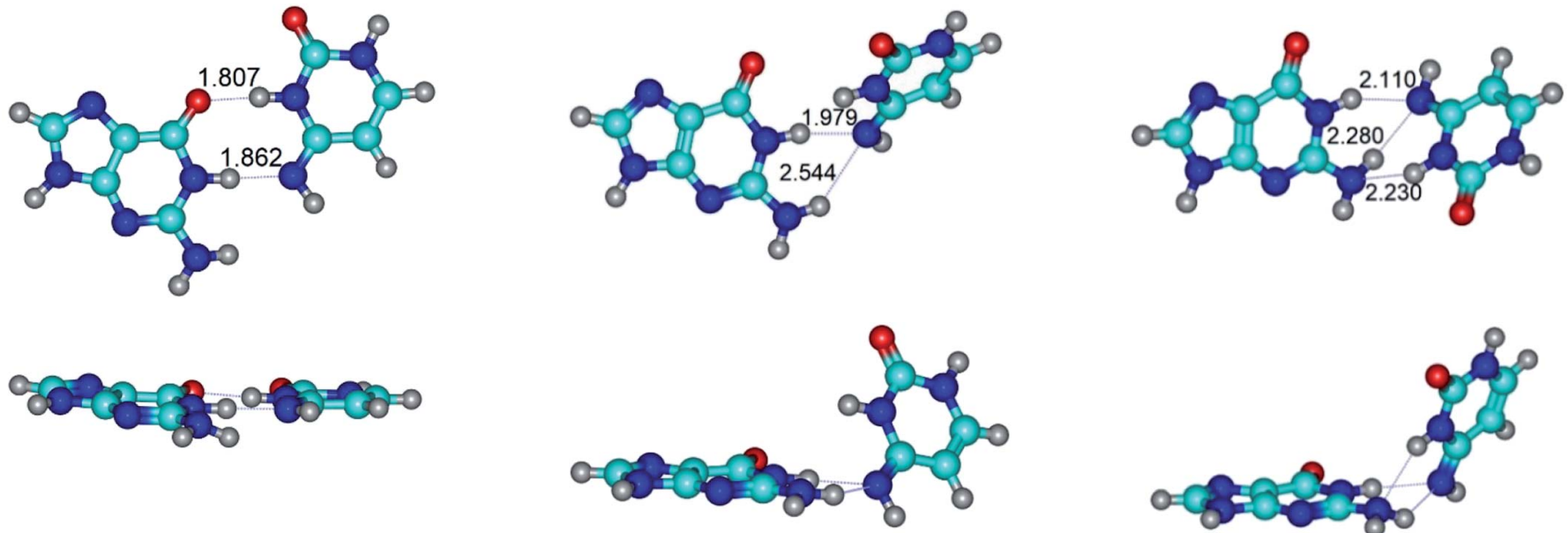

$$
\begin{gathered}
\mathrm{G} \cdot \mathrm{C}^{*}\left(\mathrm{rw}_{\mathrm{WC}}\right)_{\uparrow} \\
(\Delta G=0.00 / \Delta E=0.00 / \\
\mu=7.74)
\end{gathered}
$$

$$
\begin{aligned}
& \mathrm{TS}_{\mathrm{G}} \cdot \mathrm{C}^{*}\left(\mathrm{rw}_{\mathrm{wc}}\right)_{\uparrow} \leftrightarrow \mathrm{G} \cdot \mathrm{C}^{*}\left(\mathrm{w}_{\mathrm{wc}}\right) \\
& \left(\nu_{\mathrm{i}}=37.4 i \mathrm{~cm}^{-1}\right) \\
& (\Delta G=4.34 / \Delta E=5.80 \text { ) } \\
& \mu=8.68)
\end{aligned}
$$

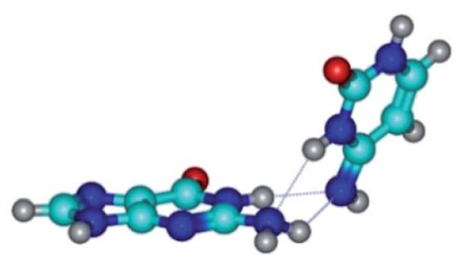

$\mathrm{G} \cdot \mathrm{C} *\left(\mathrm{w}_{\mathrm{WC}}\right)$

$(\Delta G=2.75 / \Delta E=3.05 / \mu=7.36)$

$\mathbf{G}^{*} \cdot \mathbf{C}\left(\mathbf{r w}_{\mathbf{W C}}\right)_{\uparrow} \leftrightarrow \mathbf{G}^{*} \cdot \mathbf{C}\left(\mathbf{w}_{\mathbf{W C} / \mathrm{H}}\right)$ \{rotation around the upper $\mathbf{O 6 H}^{\cdots} \cdot \mathbf{N} 3$ H-bond
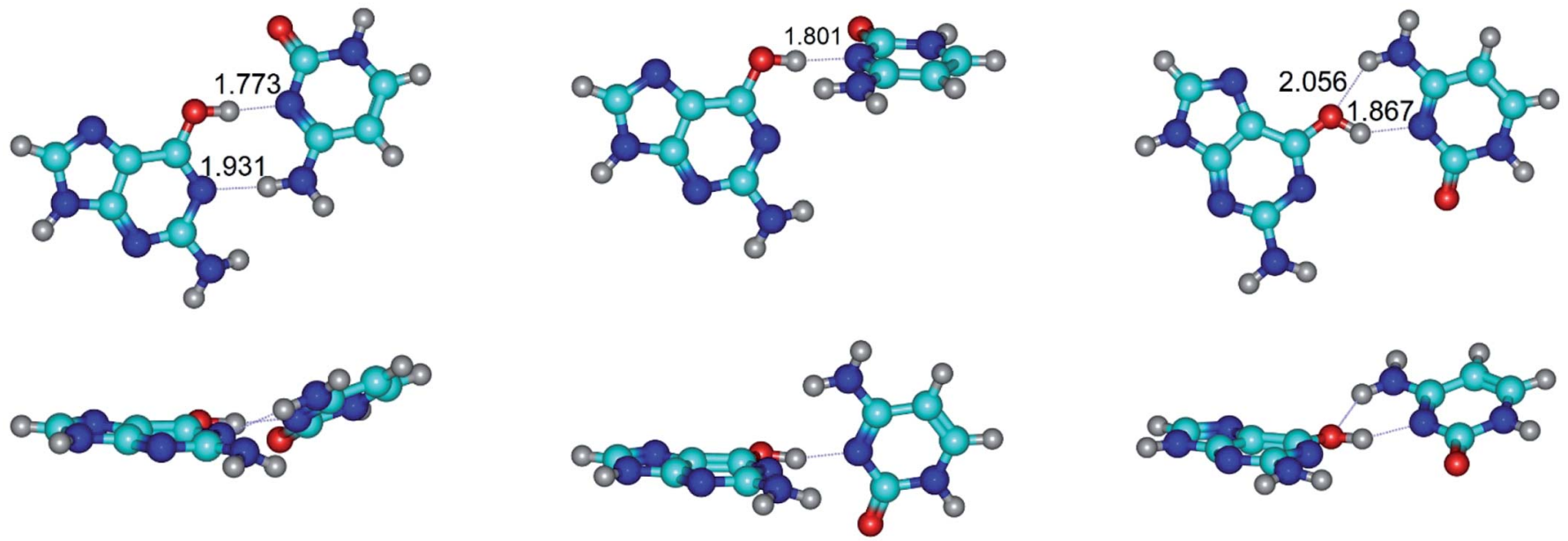

$\mathrm{G}^{*} \cdot \mathrm{C}\left(\mathrm{rw}_{\mathrm{WC}}\right)_{\uparrow}$

$(\Delta G=0.00 / \Delta E=0.00 /$

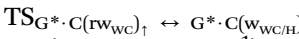

$$
\begin{aligned}
& \left(\nu_{\mathrm{i}}=38.1 i \mathrm{~cm}^{-1}\right) \\
& (\Delta G=3.73 / \Delta E=4.71 / \\
& \mu=7.39 \text { ) }
\end{aligned}
$$

$$
\begin{gathered}
\mathrm{G}^{*} \cdot \mathrm{C}\left(\mathrm{w}_{\mathrm{WC} / \mathrm{H}}\right) \\
(\Delta G=2.29 / \Delta E=4.06 / \mu=4.79)
\end{gathered}
$$

$\mathbf{G}^{*} \cdot \mathbf{C}\left(\mathbf{r w}_{\mathbf{W C}}\right)_{\uparrow} \leftrightarrow \mathbf{G}^{*} \cdot \mathbf{C}\left(\mathbf{w}_{\mathbf{W C}}\right)_{\downarrow}\{$ rotation around the lower $\mathbf{N} 4 \mathbf{H} \cdots \mathbf{N} 1 \mathrm{H}-$ bond $\}$
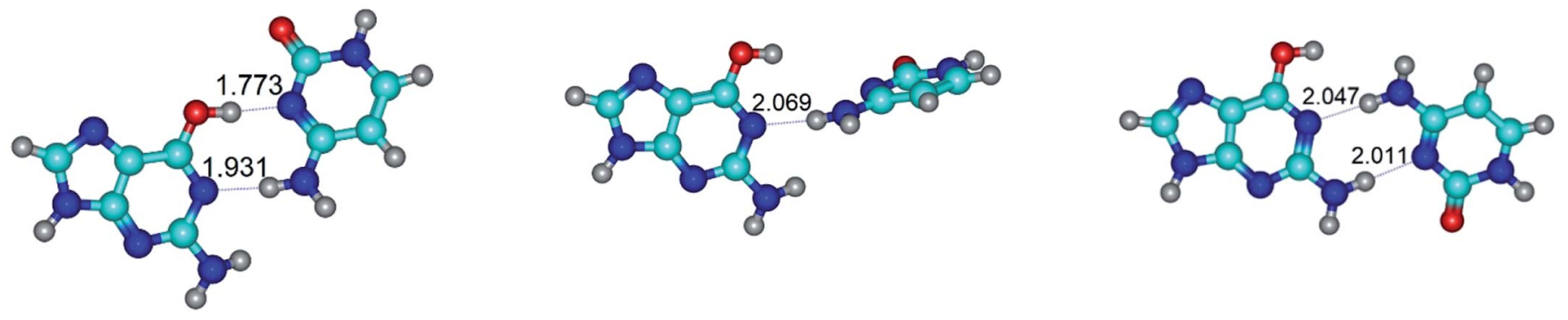

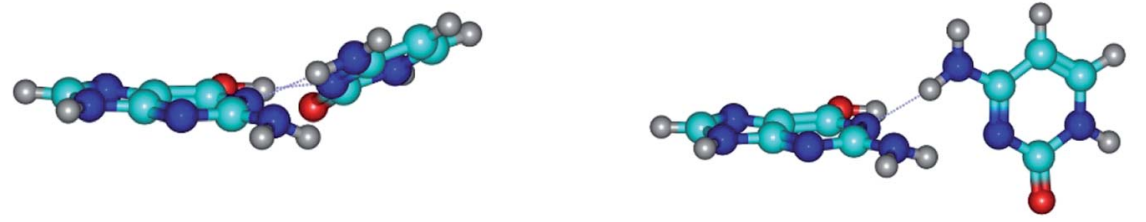

$$
\begin{gathered}
\mathrm{G}^{*} \cdot \mathrm{C}\left(\mathrm{rw}_{\mathrm{WC}}\right)_{\uparrow} \\
(\Delta G=0.00 / \Delta E=0.00 / \\
\mu=7.36)
\end{gathered}
$$

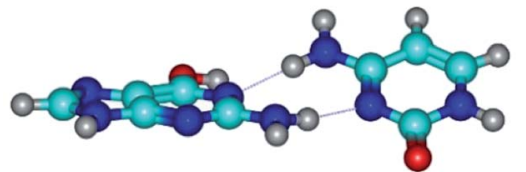

$\mathrm{G}^{*} \cdot \mathrm{C}\left(\mathrm{W}_{\mathrm{WC}}\right)_{\downarrow}$

$(\Delta G=3.32 / \Delta E=4.09 / \mu=4.70)$

$\mathbf{G}^{*} \cdot \mathbf{C}\left(\mathbf{w}_{\mathbf{W C}}\right)_{\downarrow} \leftrightarrow \mathbf{G}^{*} \cdot \mathbf{C}\left(\mathbf{r w}_{\mathrm{WC}}\right)$ \{rotation around the lower $\mathbf{N} 2 \mathbf{H}^{\cdots} \cdot \mathbf{N} 1 \mathrm{H}-$-bond
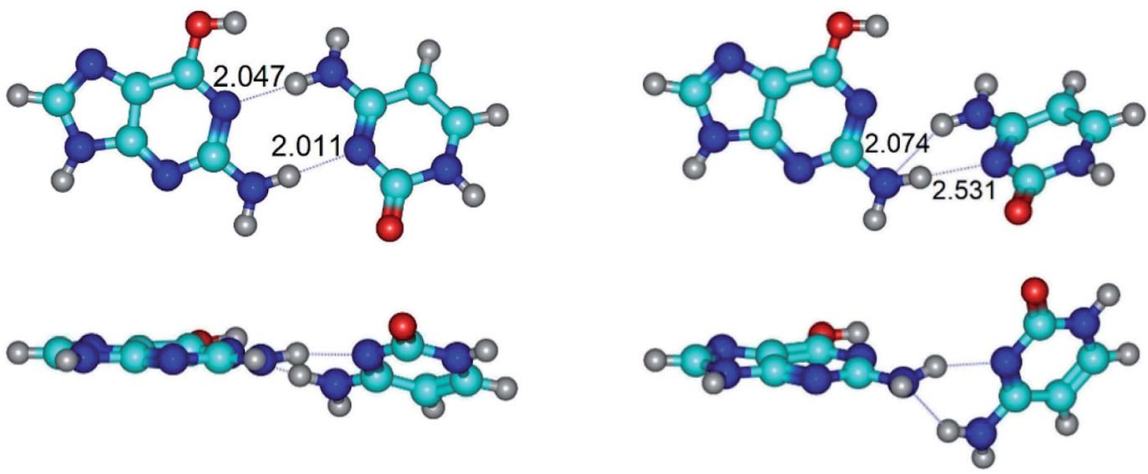

$$
\begin{gathered}
\mathrm{G}^{*} \cdot \mathrm{C}\left(\mathrm{w}_{\mathrm{WC}}\right)_{\downarrow} \\
(\Delta G=0.00 / \Delta E=0.00 / \\
\mu=4.70)
\end{gathered}
$$

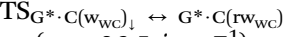

$$
\begin{aligned}
& \left(\nu_{\mathrm{i}}=36.5 i \mathrm{~cm}^{-1}\right) \\
& (\Delta G=2.96 / \Delta E=2.91 / \\
& \mu=5.63 \text { ) }
\end{aligned}
$$

$\mathrm{G}^{*} \cdot \mathrm{C}^{*} \mathrm{O} 2_{2}\left(\mathbf{w}_{\mathrm{WC}}\right)_{\uparrow} \leftrightarrow \mathrm{G}^{*} \cdot \mathrm{C}^{*}{ }_{\mathrm{O} 2}\left(\mathrm{rw}_{\mathrm{WC}}\right)_{\downarrow}\{$ rotation around the lower $\mathrm{O} 2 \mathrm{H} \cdots \mathrm{N} 1 \mathrm{H}-\mathrm{bond}\}$
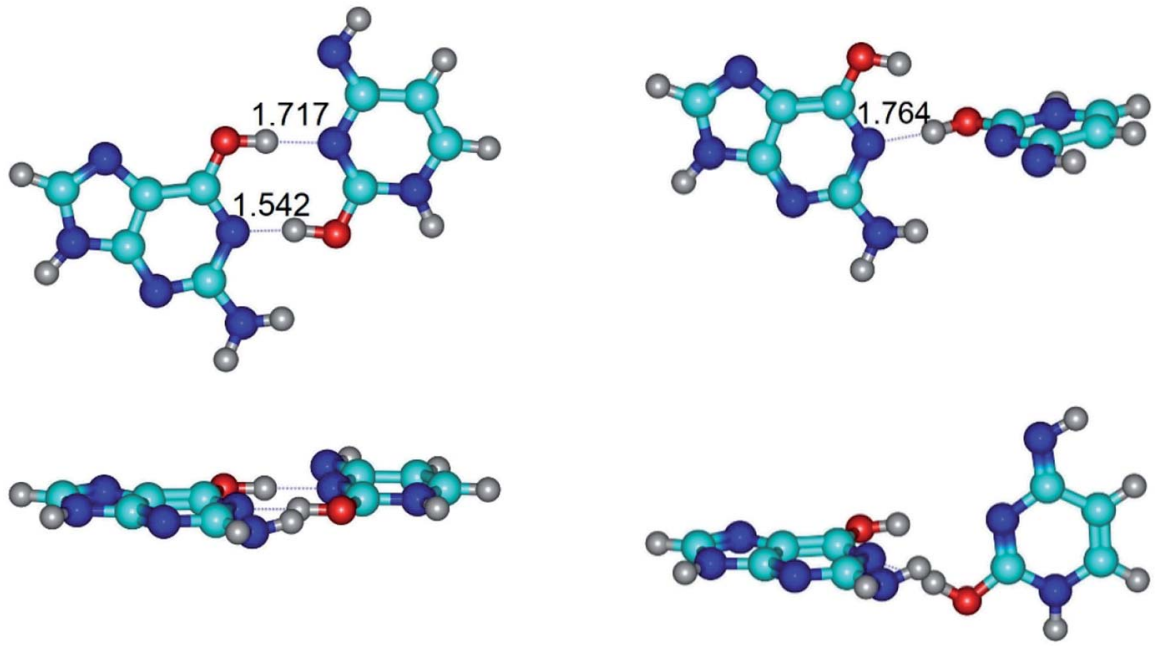

$$
\begin{gathered}
\mathrm{G}^{*} \cdot \mathrm{C}^{*}{ }_{\mathrm{O} 2}\left(\mathrm{w}_{\mathrm{WC}}\right)_{\uparrow} \\
(\Delta G=0.00 / \Delta E=0.00 / \\
\mu=6.67)
\end{gathered}
$$

$$
\begin{gathered}
\mathrm{TS}_{\mathrm{G}^{*} \cdot \mathrm{C}^{*} \mathrm{O} 2\left(\mathrm{w}_{\mathrm{wc}}\right)_{\uparrow} \leftrightarrow} \\
\mathrm{G}^{*} \cdot \mathrm{C}^{*} \mathrm{O} 2\left(\mathrm{rw}_{\mathrm{wc}}\right)_{\downarrow} \\
\left(\nu_{\mathrm{i}}=42.8 i \mathrm{~cm}^{-1}\right) \\
(\Delta G=12.20 / \Delta E=11.29 / \\
\mu=4.64)
\end{gathered}
$$
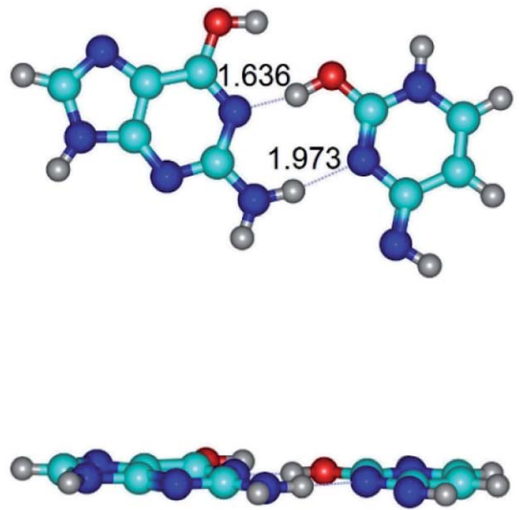

$\mathrm{G}^{*} \cdot \mathrm{C}^{*}{ }_{\mathrm{O} 2}\left(\mathrm{rw}_{\mathrm{WC}}\right)$

$(\Delta G=3.09 / \Delta E=3.82 / \mu=2.64)$ 
$\mathrm{G}^{*} \cdot \mathrm{C}^{*}{ }_{\mathrm{O} 2}\left(\mathbf{w}_{\mathrm{WC}}\right)_{\uparrow} \leftrightarrow \mathrm{G}^{*} \cdot \mathrm{C}^{*}{ }_{\mathrm{O} 2}\left(\mathrm{rw}_{\mathrm{WC} / \mathrm{H}}\right)\{$ rotation around the upper $\mathrm{O6H} \cdots \mathrm{N} 3 \mathrm{H}-\mathrm{bond}\}$
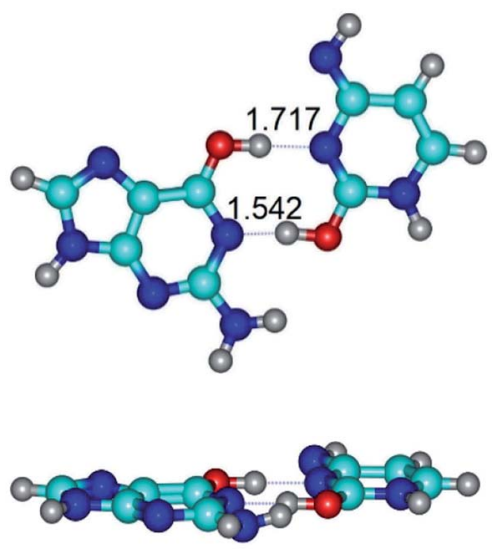

$$
\begin{gathered}
\mathrm{G}^{*} \cdot \mathrm{C}^{*}{ }_{\mathrm{O} 2}\left(\mathrm{w}_{\mathrm{WC}}\right)_{\uparrow} \\
(\Delta G=0.00 / \Delta E=0.00 / \\
\mu=6.67)
\end{gathered}
$$
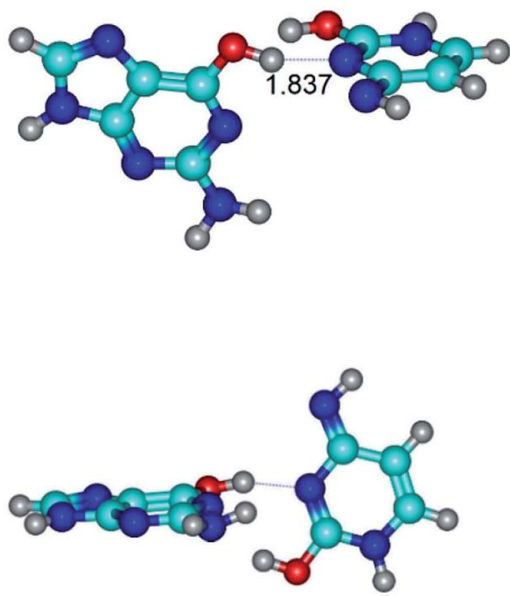

$$
\begin{gathered}
\mathrm{TS}_{\mathrm{G}^{*} \cdot \mathrm{C}^{*} \mathrm{O} 2\left(\mathrm{w}_{\mathrm{wc}}\right)_{\uparrow} \leftrightarrow} \\
\mathrm{G}^{*} \cdot \mathrm{C}^{*} \mathrm{O} 2\left(\mathrm{rw}_{\mathrm{wC} / H}\right) \\
\left(\nu_{\mathrm{i}}=55.3 i \mathrm{~cm}^{-1}\right) \\
(\Delta G=11.52 / \Delta E=11.00 / \\
\mu=6.00)
\end{gathered}
$$
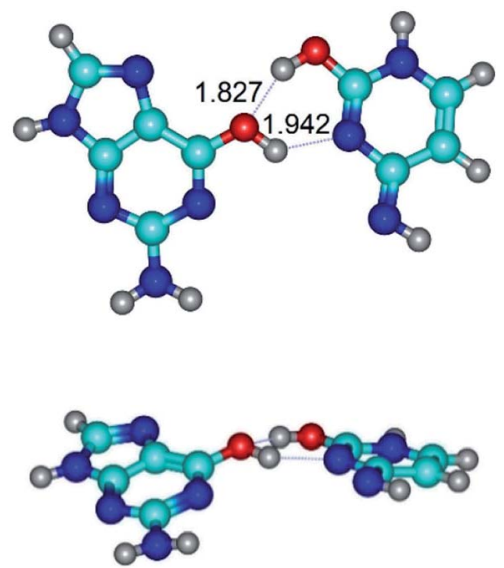

$\mathrm{G}^{*} \cdot \mathrm{C}^{*}{ }_{\mathrm{O} 2}\left(\mathrm{rw}_{\mathrm{WC} / \mathrm{H}}\right)$

$(\Delta G=8.74 / \Delta E=9.01 / \mu=3.05)$

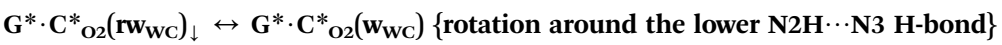
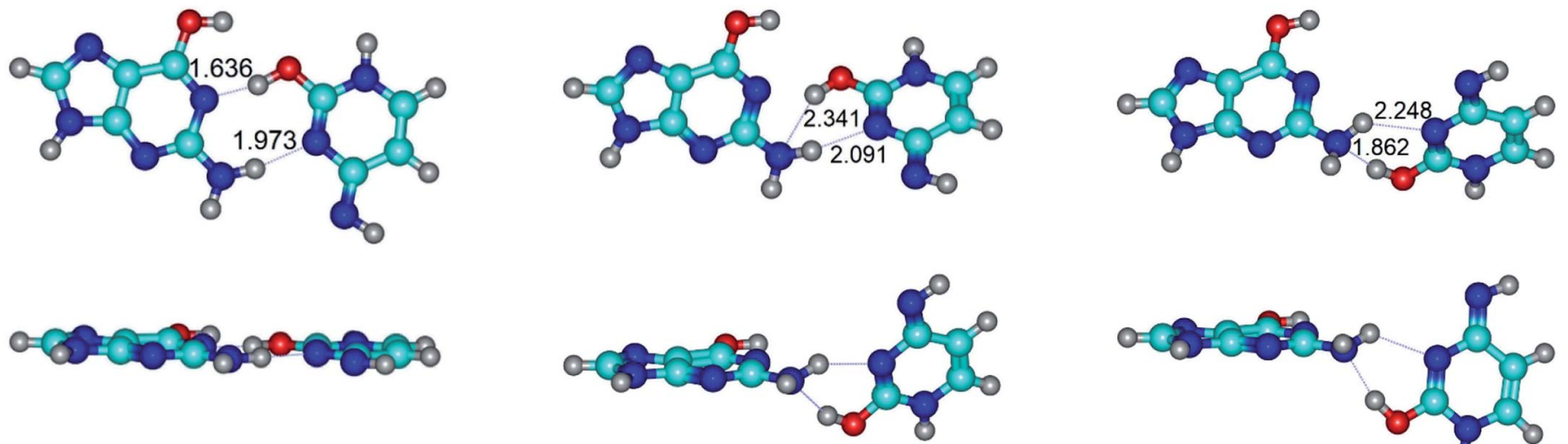

$$
\begin{gathered}
\mathrm{G}^{*} \cdot \mathrm{C}^{*}{ }_{\mathrm{O} 2}\left(\mathrm{rw}_{\mathrm{WC}}\right)_{\downarrow} \\
(\Delta G=0.00 / \Delta E=0.00 / \\
\mu=2.64)
\end{gathered}
$$

$$
\begin{gathered}
\mathrm{TS}_{\mathrm{G}^{*} \cdot \mathrm{C}^{*} \mathrm{O} 2\left(\mathrm{rw}_{\mathrm{wc}}\right)_{\downarrow} \leftrightarrow} \\
\mathrm{G}^{*} \cdot \mathrm{C}^{*} \mathrm{O} 2\left(\mathrm{w}_{\mathrm{wc}}\right) \\
\left(\nu_{\mathrm{i}}=87.3 i \mathrm{~cm}^{-1}\right) \\
(\Delta G=9.10 / \Delta E=7.84 / \\
\mu=4.48)
\end{gathered}
$$

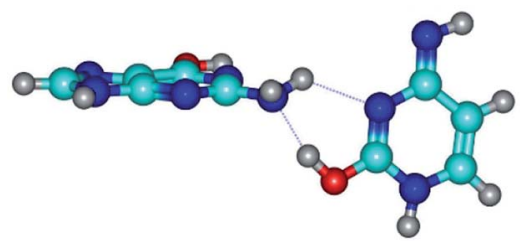

$$
\begin{gathered}
\mathrm{G}^{*} \cdot \mathrm{C}^{*}{ }_{\mathrm{O} 2}\left(\mathrm{w}_{\mathrm{WC}}\right) \\
(\Delta G=7.07 / \Delta E=6.14 / \mu=5.05)
\end{gathered}
$$

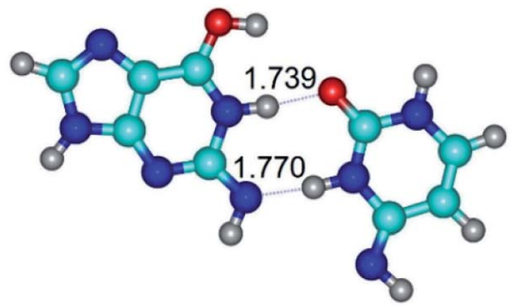



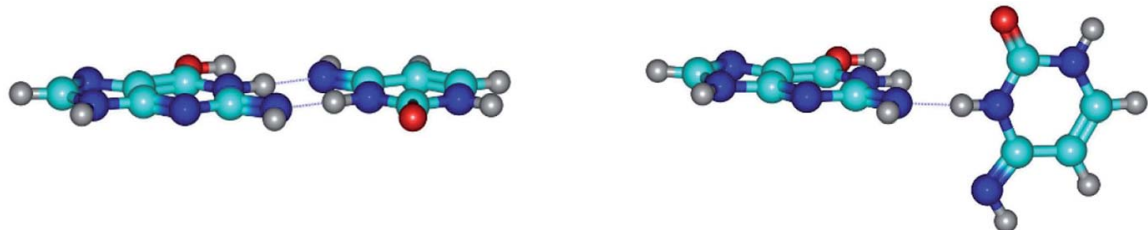

$$
\begin{gathered}
\mathrm{G}^{*}{ }_{\mathrm{N} 2} \cdot \mathrm{C}^{*}\left(\mathrm{~W}_{\mathrm{WC}}\right)_{\downarrow} \\
(\Delta G=0.00 / \Delta E=0.00 / \\
\mu=4.17)
\end{gathered}
$$

$$
\begin{gathered}
\mathrm{TS}_{\mathrm{G}^{*} \mathrm{~N} 2 \cdot \mathrm{C}^{*}\left(\mathrm{w}_{\mathrm{wC}}\right)_{\downarrow} \leftrightarrow} \\
\mathrm{G}^{*} \mathrm{~N} 2 \cdot \mathrm{C} *\left(\mathrm{rw}_{\mathrm{wc}}\right)_{\downarrow} \\
\left(\nu_{\mathrm{i}}=36.5 i \mathrm{~cm}^{-1}\right) \\
(\Delta G=13.90 / \Delta E=15.15 / \\
\mu=2.62)
\end{gathered}
$$

$\mathbf{G}^{* \mathrm{t}} \cdot \mathbf{C}^{*}{ }_{\mathrm{O} 2}\left(\mathrm{rw}_{\mathrm{WC}}\right)_{\downarrow} \leftrightarrow \mathbf{G}^{* \mathrm{t}} \cdot \mathbf{C}^{*}{ }_{\mathrm{O} 2}\left(\mathbf{w}_{\mathrm{WC}}\right)\{$ rotation around the lower $\mathbf{N} 2 \mathrm{H} \cdots \mathbf{N} 3 \mathrm{H}-\mathrm{bond}\}$
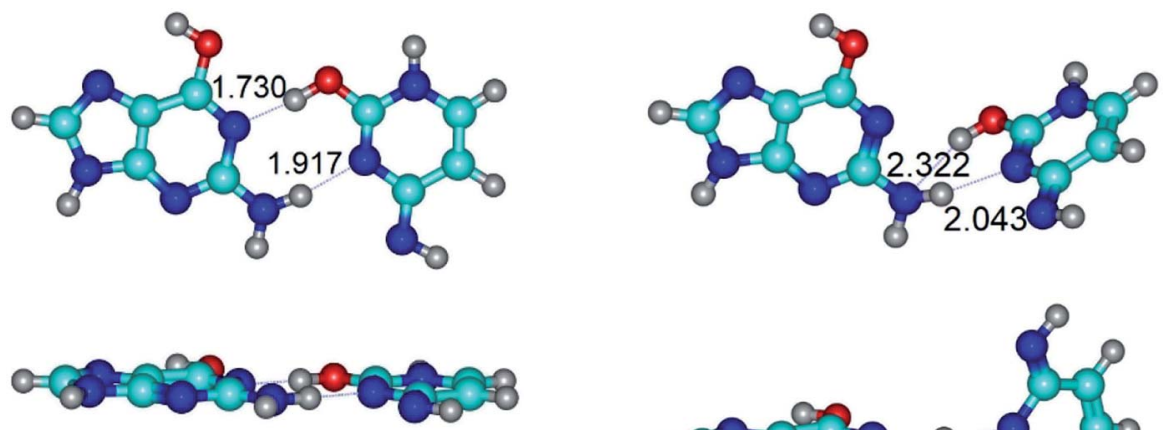

$$
\begin{gathered}
\mathrm{G}^{* \mathrm{t}} \cdot \mathrm{C}^{*}{ }_{\mathrm{O} 2}\left(\mathrm{rw}_{\mathrm{WC}}\right)_{\downarrow} \\
(\Delta G=0.00 / \Delta E=0.00 / \\
\mu=2.25)
\end{gathered}
$$

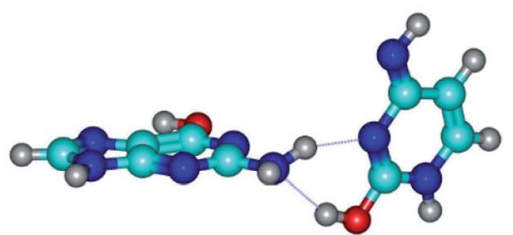

$$
\begin{gathered}
\mathrm{TS}_{\mathrm{G}^{*} \mathrm{t} \cdot \mathrm{C}^{*} \mathrm{O} 2\left(\mathrm{rw}_{\mathrm{wC}}\right) \downarrow} \\
\mathrm{G}^{*} \mathrm{t} \cdot \mathrm{C}^{*} \mathrm{O} 2\left(\mathrm{w}_{\mathrm{wc}}\right) \\
\left(\nu_{\mathrm{i}}=72.6 i \mathrm{~cm}^{-1}\right) \\
(\Delta G=9.55 / \Delta E=9.27 / \\
\mu=1.75)
\end{gathered}
$$

$\mathbf{G}^{*} \cdot \mathbf{C}^{* \mathrm{t}}{ }_{\mathrm{O} 2}\left(\mathbf{w}_{\mathrm{WC}}\right)_{\uparrow} \leftrightarrow \mathrm{G}^{*} \cdot \mathrm{C}^{* \mathrm{t}}{ }_{\mathrm{O} 2}\left(\mathrm{rW}_{\mathrm{WC} / \mathrm{H}}\right)$ \{rotation around the upper O6H $\cdots \mathrm{N} 3 \mathrm{H}-$ bond
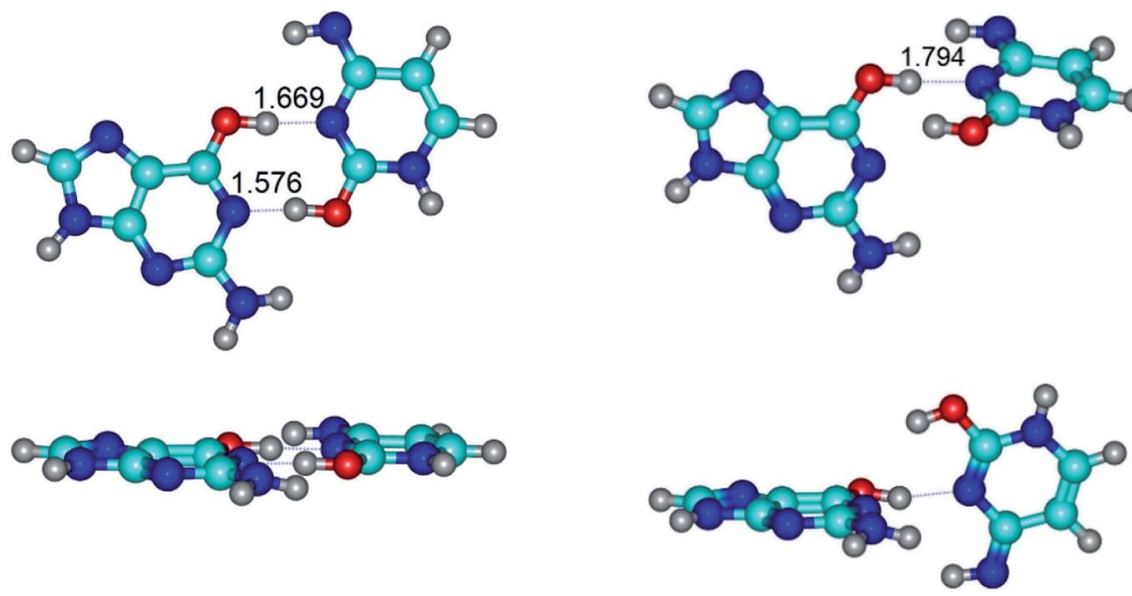

$$
\begin{gathered}
\mathrm{G}^{*} \cdot \mathrm{C}^{* \mathrm{t}}{ }_{\mathrm{O} 2}\left(\mathrm{~W}_{\mathrm{WC}}\right)_{\uparrow} \\
(\Delta G=0.00 / \Delta E=0.00 / \\
\mu=7.38)
\end{gathered}
$$
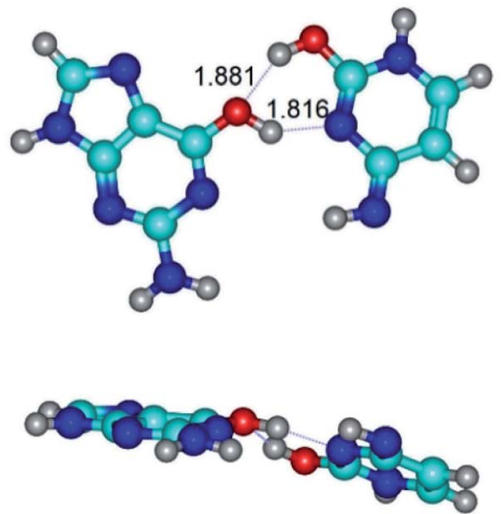

$$
\begin{gathered}
\mathrm{G}^{*} \cdot \mathrm{C}^{* \mathrm{O}^{\mathrm{O}}\left(\mathrm{rw}_{\mathrm{WC} / \mathrm{H}}\right)} \\
(\Delta G=8.10 / \Delta E=8.45 / \mu=3.71)
\end{gathered}
$$


Table 1 (Contd.)

$\mathbf{G}^{*} \cdot \mathbf{C}^{* \mathrm{t}}{ }_{\mathrm{O} 2}\left(\mathbf{w}_{\mathrm{WC}}\right)_{\uparrow} \leftrightarrow \mathbf{G}^{*} \cdot \mathbf{C}^{* \mathrm{t}}{ }_{\mathrm{O} 2}\left(\mathbf{r w}_{\mathrm{WC}}\right)_{\downarrow}$ \{rotation around the lower $\left.\mathrm{O} 2 \mathrm{H}^{\cdots} \cdot \mathbf{N} 1 \mathrm{H}-\mathrm{bond}\right\}$
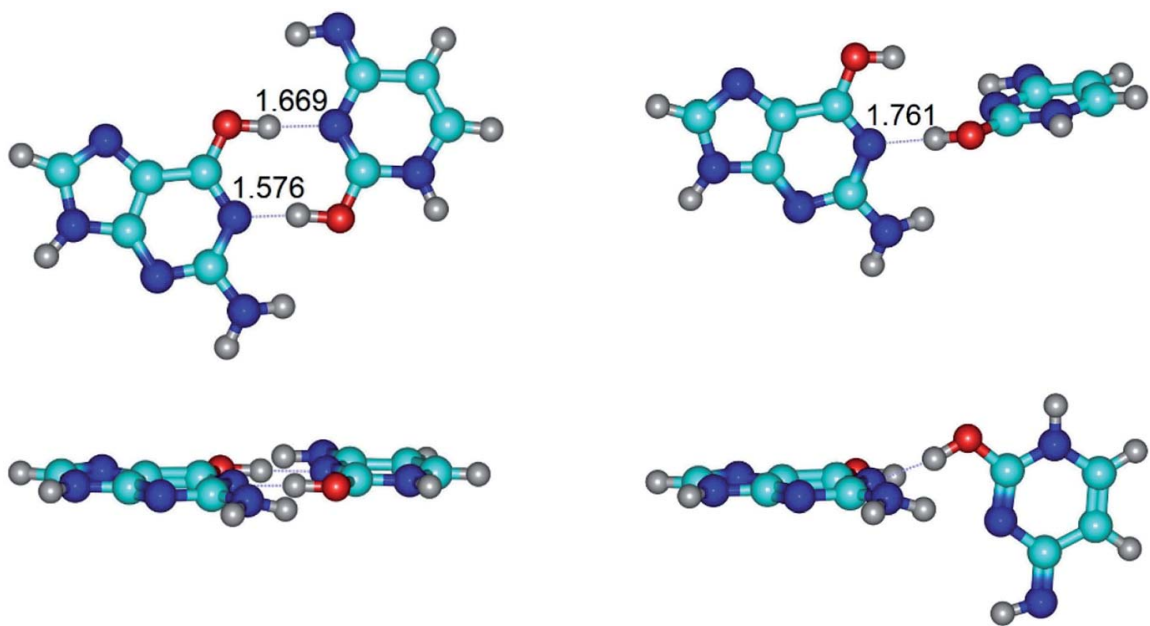

$$
\begin{gathered}
\mathrm{G}^{*} \cdot \mathrm{C}^{* \mathrm{t}}{ }_{\mathrm{O} 2}\left(\mathrm{w}_{\mathrm{WC}}\right)_{\uparrow} \\
(\Delta G=0.00 / \Delta E=0.00 / \\
\mu=7.38)
\end{gathered}
$$

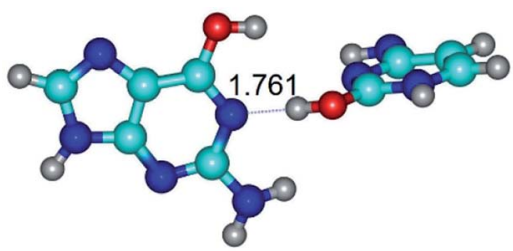

$$
\begin{gathered}
\mathrm{TS}_{\mathrm{G}^{*} \cdot \mathrm{C}^{*} \mathrm{tO} 2\left(\mathrm{w}_{\mathrm{wc}}\right)_{\uparrow} \leftrightarrow} \\
\mathrm{G}^{*} \cdot \mathrm{C}^{*} \mathrm{tO} 2\left(\mathrm{rw}_{\mathrm{wc}}\right)_{\downarrow} \\
\left(\nu_{\mathrm{i}}=39.5 i \mathrm{~cm}^{-1}\right) \\
(\Delta G=11.27 / \Delta E=10.80 / \\
\mu=5.74)
\end{gathered}
$$

$\mathbf{G}^{*} \cdot \mathrm{C}^{* \mathrm{t}}{ }_{\mathrm{O} 2}\left(\mathrm{rw}_{\mathrm{WC}}\right)_{\downarrow} \leftrightarrow \mathbf{G}^{*} \cdot \mathbf{C}^{* \mathrm{t}}{ }_{\mathrm{O} 2}\left(\mathbf{w}_{\mathrm{WC}}\right)$ \{rotation around the lower N2H $\cdots \mathbf{N} 3 \mathrm{H}$-bond
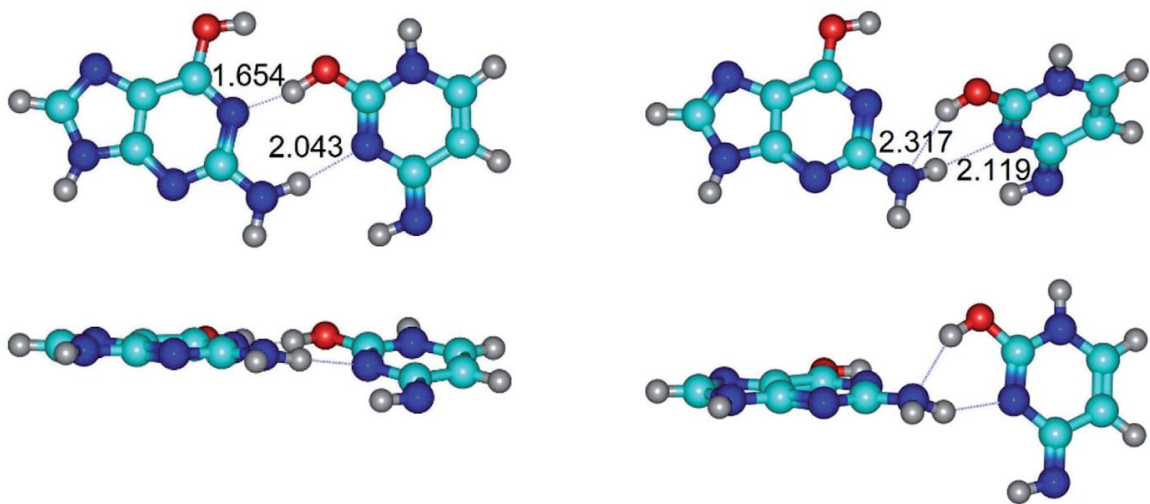

$$
\begin{gathered}
\mathrm{G}^{*} \cdot \mathrm{C}^{* \mathrm{t}}{ }_{\mathrm{O} 2}\left(\mathrm{rw}_{\mathrm{WC}}\right)_{\downarrow} \\
(\Delta G=0.00 / \Delta E=0.00 / \\
\mu=2.34)
\end{gathered}
$$

$$
\begin{gathered}
\mathrm{TS}_{\mathrm{G}^{*} \cdot \mathrm{C}^{*} \mathrm{tO} 2\left(\mathrm{rw}_{\mathrm{wc}}\right)_{\downarrow} \leftrightarrow} \\
\mathrm{G}^{*} \cdot \mathrm{C}^{*} \mathrm{tO} 2\left(\mathrm{w}_{\mathrm{wc}}\right) \\
\left(\nu_{\mathrm{i}}=101.9 i \mathrm{~cm}^{-1}\right) \\
(\Delta G=6.87 / \Delta E=6.58 / \\
\mu=4.03)
\end{gathered}
$$

$\mathrm{G}^{* \mathrm{t}} \cdot \mathrm{C}^{* \mathrm{t}}{ }_{\mathrm{O} 2}\left(\mathrm{rw}_{\mathrm{WC}}\right)_{\downarrow} \leftrightarrow \mathrm{G}^{* \mathrm{t}} \cdot \mathrm{C}^{* \mathrm{t}}{ }_{\mathrm{O} 2}\left(\mathrm{w}_{\mathrm{WC}}\right)$ \{rotation around the lower N2H $\left.\cdots \mathrm{N} 3 \mathrm{H}-\mathrm{bond}\right\}$
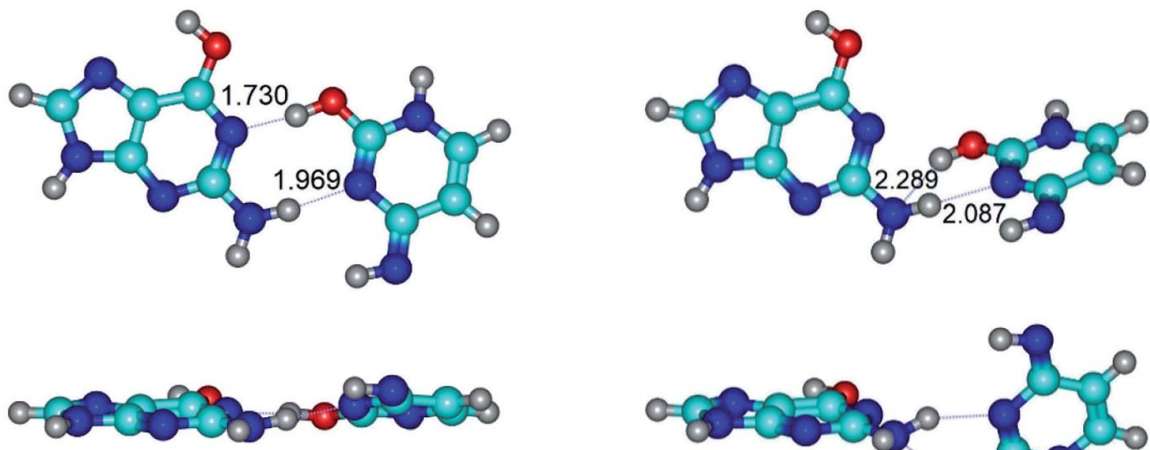
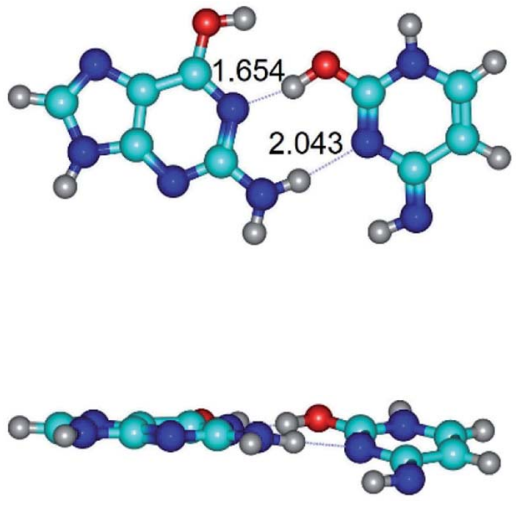

$$
\begin{gathered}
\mathrm{G}^{*} \cdot \mathrm{C}^{* \mathrm{t}} \mathrm{O} 2\left(\mathrm{rw}_{\mathrm{WC}}\right)_{\downarrow} \\
(\Delta G=5.73 / \Delta E=5.65 / \mu=2.34)
\end{gathered}
$$
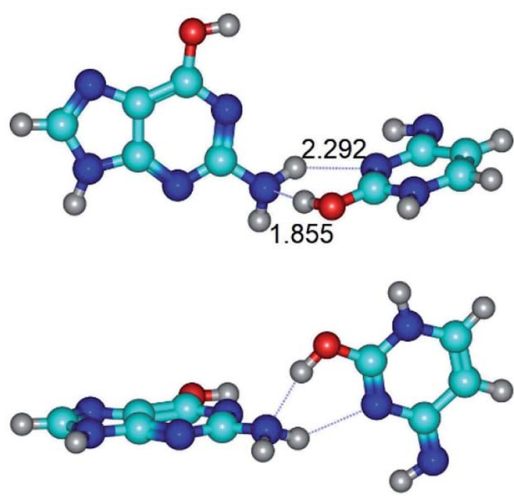

$$
\begin{gathered}
\mathrm{G}^{*} \cdot \mathrm{C}^{* \mathrm{t}} \mathrm{o}_{2}\left(\mathrm{~W}_{\mathrm{WC}}\right) \\
(\Delta G=4.58 / \Delta E=4.75 / \mu=5.53)
\end{gathered}
$$
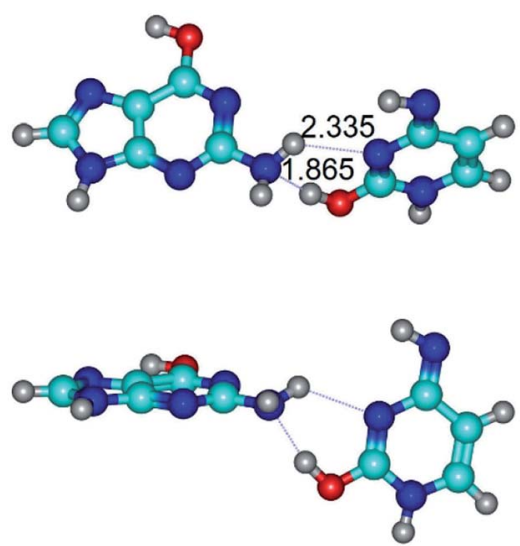
Table 1 (Contd.)

$$
\begin{array}{cc}
\mathrm{G}^{* \mathrm{t}} \cdot \mathrm{C}^{* \mathrm{t}}{ }_{\mathrm{O} 2}\left(\mathrm{rw}_{\mathrm{WC}}\right)_{\downarrow} & \mathrm{TS}_{\mathrm{G}^{*} \mathrm{t} \cdot \mathrm{C}^{*} \mathrm{tO} 2\left(\mathrm{rw}_{\mathrm{wC}}\right)_{\downarrow} \leftrightarrow} \\
(\Delta G=0.00 / \Delta E=0.00 / & \mathrm{G}^{*} \cdot \mathrm{C}^{*} \mathrm{tO} 2\left(\mathrm{w}_{\mathrm{wC}}\right) \\
\mu=4.88) & \left(\nu_{\mathrm{i}}=92.9 i \mathrm{~cm}^{-1}\right) \\
& (\Delta G=7.82 / \Delta E=7.96 / \\
& \mu=4.60)
\end{array}
$$

$\mathbf{G}^{* \mathrm{t}}{ }_{\mathbf{N} 2} \cdot \mathbf{C}^{*}\left(\mathbf{w}_{\mathbf{W C}}\right)_{\downarrow} \leftrightarrow \mathbf{G}^{*{ }^{\mathrm{t}} \mathbf{N} 2} \cdot \mathbf{C}^{*}\left(\mathbf{r w}_{\mathbf{W C}}\right)_{\downarrow}\{$ rotation around the lower $\mathbf{N} 3 \mathbf{H} \cdots \mathbf{N} 2$ H-bond $\}$
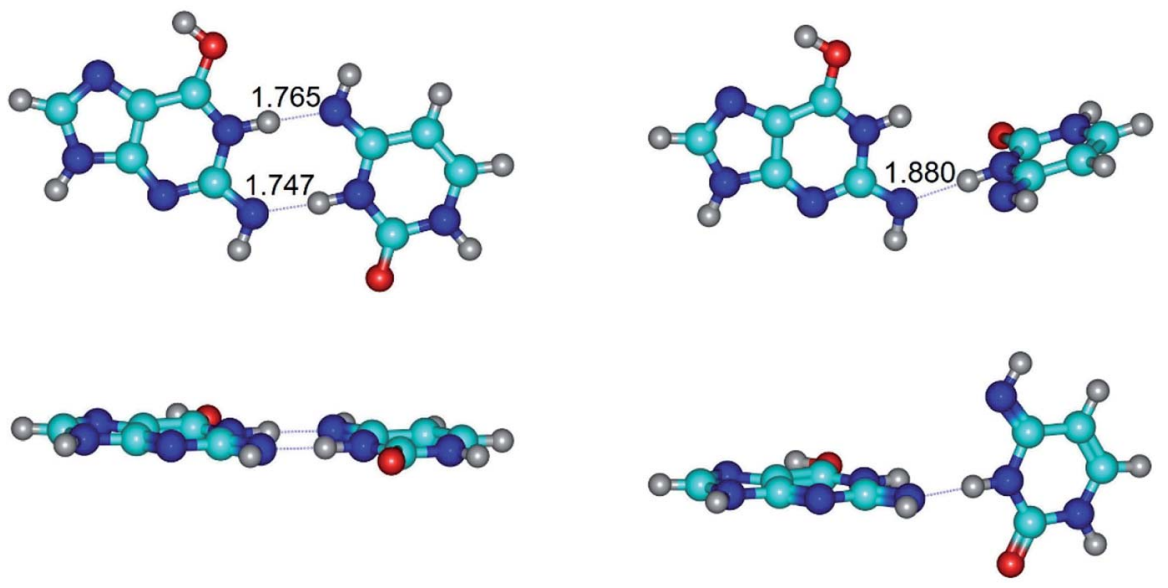

$$
\begin{gathered}
\mathrm{G}^{* \mathrm{t}}{ }_{\mathrm{N} 2} \cdot \mathrm{C}^{*}\left(\mathrm{w}_{\mathrm{WC}}\right)_{\downarrow} \\
(\Delta G=0.00 / \Delta E=0.00 / \\
\mu=3.78)
\end{gathered}
$$

$$
\begin{gathered}
\mathrm{TS}_{\mathrm{G}^{*} \mathrm{tN} 2 \cdot \mathrm{C} *\left(\mathrm{w}_{\mathrm{Wc}}\right)_{\downarrow} \leftrightarrow} \\
\mathrm{G}^{*} \mathrm{tN} 2 \cdot \mathrm{C}^{*}\left(\mathrm{rw}_{\mathrm{wc}}\right)_{\downarrow} \\
\left(\nu_{\mathrm{i}}=28.3 i \mathrm{~cm}^{-1}\right) \\
(\Delta G=11.90 / \Delta E=13.01 / \\
\mu=3.01)
\end{gathered}
$$

$\mathbf{G}^{* \mathrm{t}}{ }_{\mathbf{N} 2} \cdot \mathbf{C}^{*}\left(\mathbf{r w}_{\mathbf{W C}}\right)_{\downarrow} \leftrightarrow \mathbf{G}^{* \mathrm{t}}{ }_{\mathbf{N} 2} \cdot \mathbf{C}^{*}\left(\mathbf{w}_{\mathbf{W C}}\right)_{\uparrow}$ \{rotation around the upper $\mathbf{N} 1 H^{\cdots} \mathbf{O} 2$ H-bond
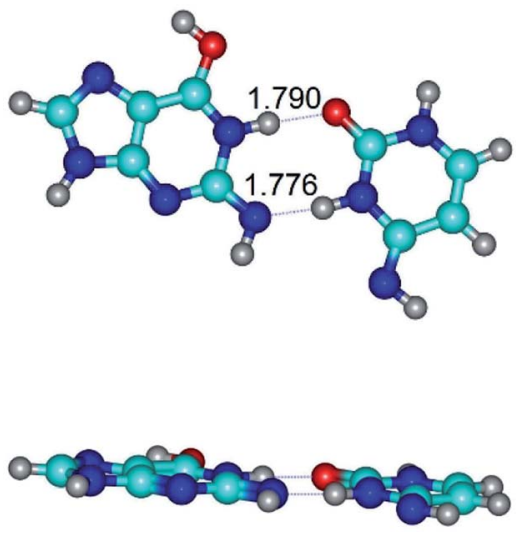

$$
\begin{gathered}
\mathrm{G}^{* \mathrm{t}}{ }_{\mathrm{N} 2} \cdot \mathrm{C}^{*}\left(\mathrm{rw}_{\mathrm{WC}}\right)_{\downarrow} \\
(\Delta G=0.00 / \Delta E=0.00 / \\
\mu=2.79)
\end{gathered}
$$
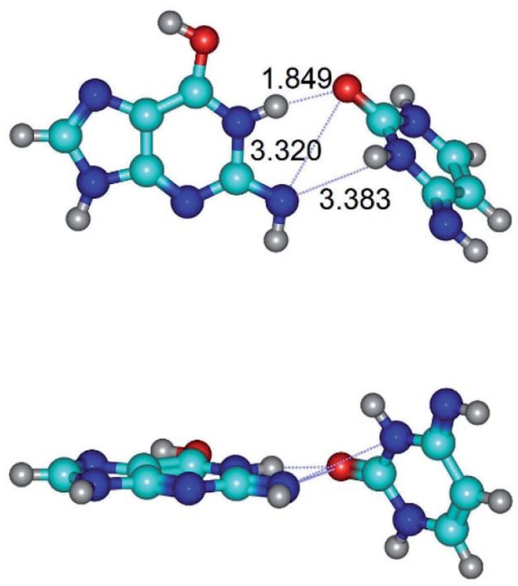

$\mathrm{TS}_{\mathrm{G}} * \mathrm{tN} 2 \cdot \mathrm{C}^{*}\left(\mathrm{rw}_{\mathrm{wC}}\right)_{\downarrow} \leftrightarrow$

$\mathrm{G}^{*} \mathrm{tN}^{2} \cdot \mathrm{C}^{*}\left(\mathrm{w}_{\mathrm{wC}}\right)_{+}\left(\nu_{\mathrm{i}}=36.4\right.$

$\left.i \mathrm{~cm}^{-1}\right)(\Delta G=8.06 / \Delta E=$ $8.02 / \mu=3.67)$
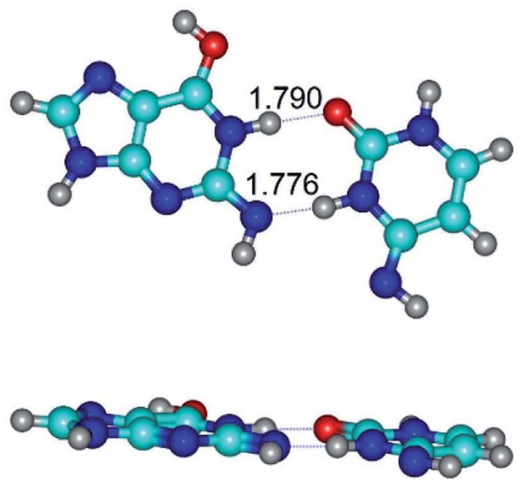

$\mathrm{G}^{* \mathrm{t}}{ }_{\mathrm{N} 2} \cdot \mathrm{C}^{*}\left(\mathrm{rw}_{\mathrm{WC}}\right)_{\downarrow}$

$(\Delta G=3.04 / \Delta E=3.02 / \mu=2.79)$
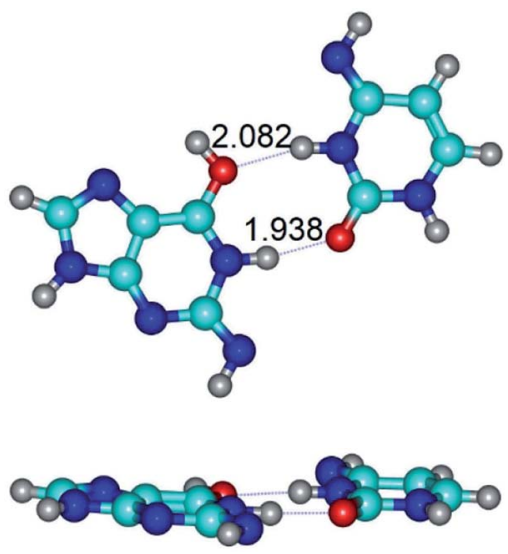

$\mathrm{G}^{* \mathrm{t}}{ }_{\mathrm{N} 2} \cdot \mathrm{C} *\left(\mathrm{w}_{\mathrm{WC}}\right) \uparrow$

$(\Delta G=7.28 / \Delta E=9.50 / \mu=4.23)$ 
Table 1 (Contd.)

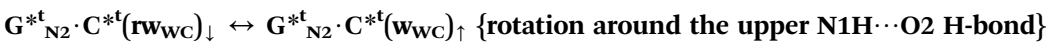
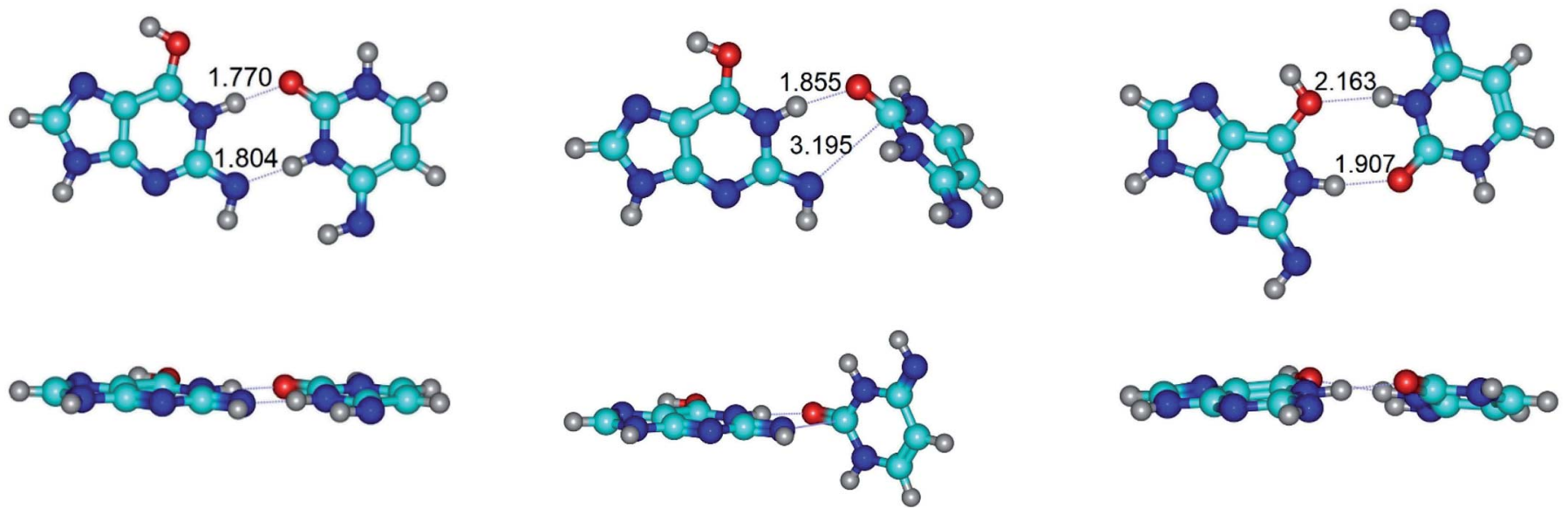

$$
\begin{gathered}
\mathrm{G}^{* \mathrm{t}}{ }_{\mathrm{N} 2} \cdot \mathrm{C}^{* \mathrm{t}}\left(\mathrm{rw}_{\mathrm{WC}}\right)_{\downarrow} \\
(\Delta G=0.00 / \Delta E=0.00 /
\end{gathered}
$$

$\mathrm{TS}_{\mathrm{G}} * \mathrm{tN} 2 \cdot \mathrm{C}^{*} \mathrm{t}\left(\mathrm{rw}_{\mathrm{wC}}\right)_{\downarrow} \leftrightarrow$

$\mathrm{G}^{*} \mathrm{tN2} \cdot \mathrm{C} * \mathrm{t}\left(\mathrm{w}_{\mathrm{WC}}\right)_{1}$

$\left(\nu_{\mathrm{i}}=28.9 i \mathrm{~cm}^{-1}\right)$

$(\Delta G=9.92 / \Delta E=9.99 /$

$$
\mu=4.01)
$$

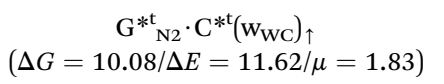

Hoogsteen $(\mathrm{H})$, reverse Hoogsteen $(\mathrm{rH})$, wobble Hoogsteen $\left(\mathrm{w}_{\mathrm{H}}\right)$ and reverse wobble Hoogsteen $\left(\mathrm{rw}_{\mathrm{H}}\right)$ configurations $\mathbf{G}^{*} \cdot \mathbf{C}^{*}(\mathbf{r H}) \leftrightarrow \mathbf{G}^{*} \cdot \mathbf{C}^{*}(\mathbf{H})$ \{rotation around the middle N3H $\cdots \mathbf{N} 7 \mathrm{H}$-bond
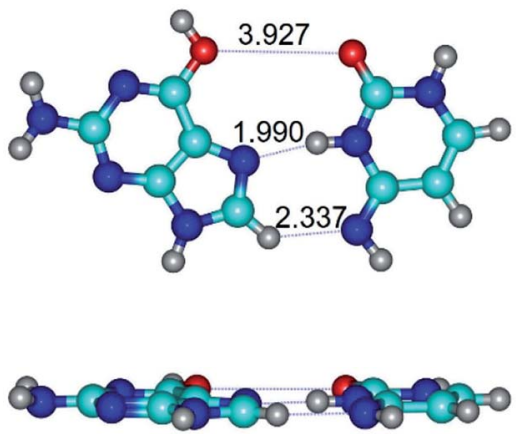

$\mathrm{G}^{*} \cdot \mathrm{C}^{*}(\mathrm{rH})$

$(\Delta G=0.00 / \Delta E=0.00 /$
$\mu=2.84)$
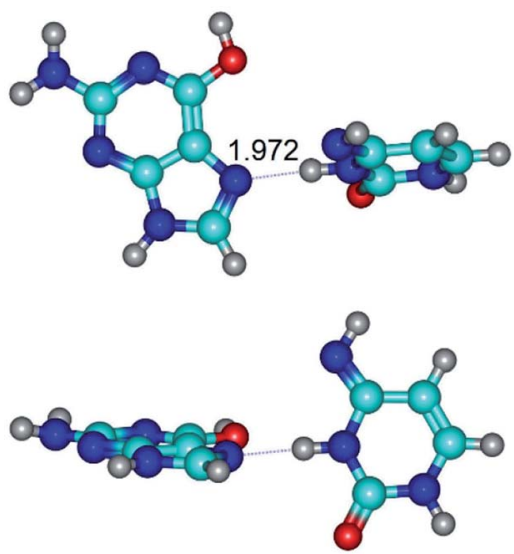

$$
\begin{gathered}
\mathrm{TS}_{\mathrm{G}^{*} \cdot \mathrm{C} *(\mathrm{H})} \leftrightarrow \mathrm{G}^{*} \cdot \mathrm{C}^{*}(\mathrm{rH}) \\
\left(\nu_{\mathrm{i}}=11.3 i \mathrm{~cm}^{-1}\right) \\
(\Delta G=3.58 / \Delta E=2.90 / \\
\mu=1.45)
\end{gathered}
$$

$\mathbf{G}^{* \mathrm{t}} \cdot \mathbf{C}^{*}(\mathrm{H}) \leftrightarrow \mathbf{G}^{* \mathrm{t}} \cdot \mathbf{C}^{*}(\mathbf{r H})\{$ rotation around the middle N3H $\cdots \mathrm{N} 7 \mathrm{H}-$ bond

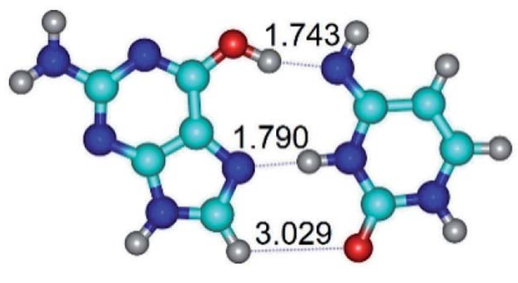

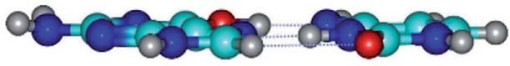
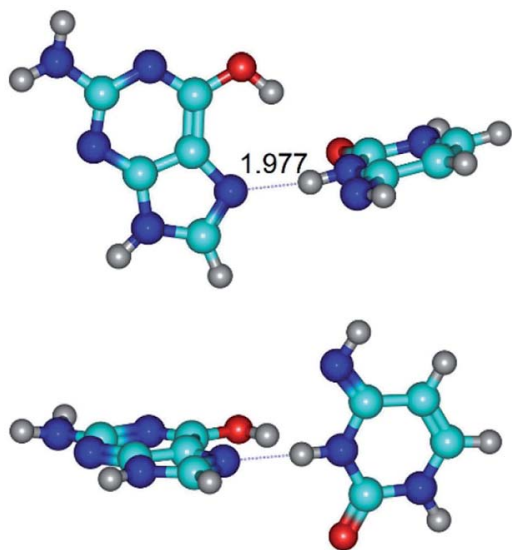
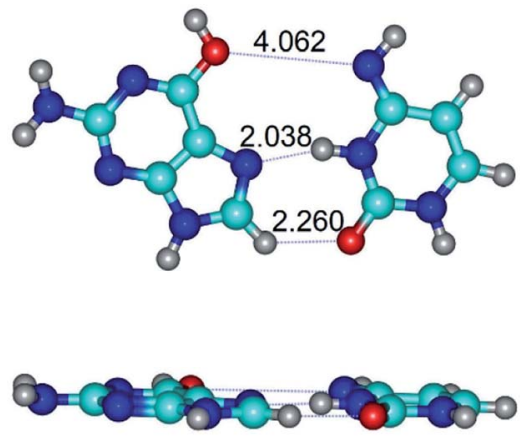

$$
(\Delta G=0.76 / \Delta E=0.75 / \mu=2.22)
$$
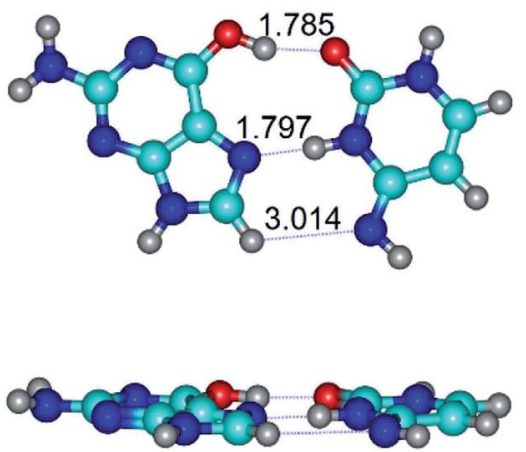
Table 1 (Contd.)

$$
\begin{array}{cc}
\mathrm{G}^{* \mathrm{t}} \cdot \mathrm{C}^{*}(\mathrm{H}) & \mathrm{TS}_{\mathrm{G} * \mathrm{t} \cdot \mathrm{C} *(\mathrm{H}) \leftrightarrow \mathrm{G}^{* \mathrm{t}} \cdot \mathrm{C}^{*}(\mathrm{rH})} \\
(\Delta G=0.00 / \Delta E=0.00 / & \left(\nu_{\mathrm{i}}=25.8 i \mathrm{~cm}^{-1}\right) \\
\mu=5.16) & (\Delta G=11.35 / \Delta E=12.71 / \\
& \mu=3.33)
\end{array}
$$$$
\mathrm{G}^{* \mathrm{t}} \cdot \mathrm{C}^{*}(\mathrm{rH})
$$$$
(\Delta G=2.59 / \Delta E=2.72 / \mu=5.43)
$$

$\mathbf{G}^{* \mathrm{t}} \cdot \mathbf{C}^{*}(\mathbf{H}) \leftrightarrow \mathbf{G}^{* \mathrm{t}} \cdot \mathbf{C}^{*}\left(\mathrm{rw}_{\mathbf{H}}\right)$ \{rotation around the upper $\left.\mathbf{0 6 H} \cdots \mathbf{N} 4 \mathrm{H}-\mathrm{bond}\right\}$
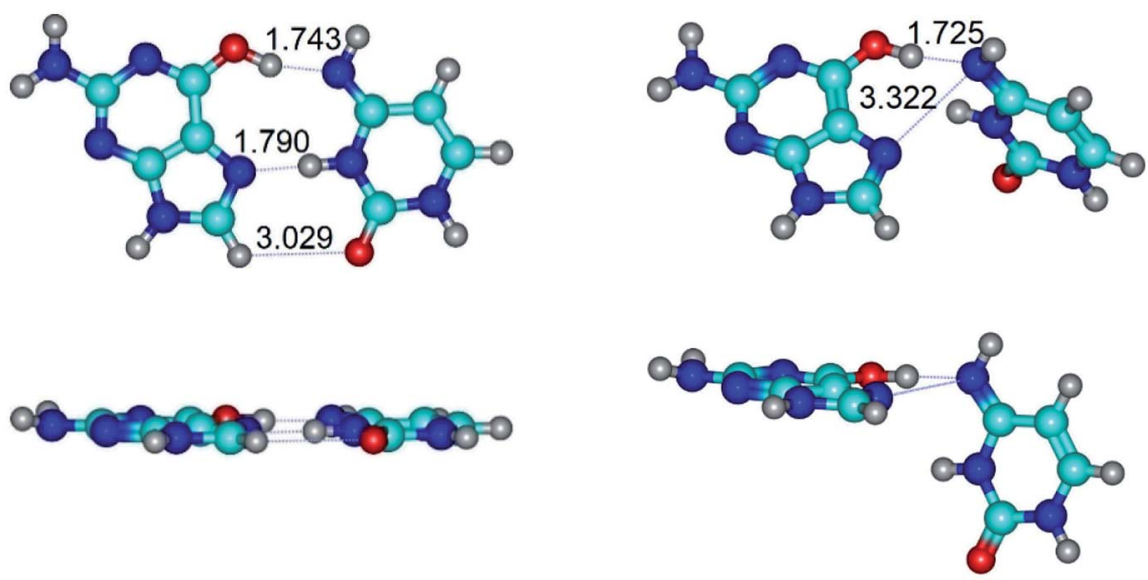

$$
\begin{array}{cc}
\mathrm{G}^{* \mathrm{t}} \cdot \mathrm{C}^{*}(\mathrm{H}) & \mathrm{TS}_{\mathrm{G}^{*} \cdot \mathrm{C}^{*}(\mathrm{H}) \leftrightarrow \mathrm{G}^{*} \cdot \mathrm{C}^{*}\left(\mathrm{rw}_{\mathrm{H}}\right)} \\
(\Delta G=0.00 / \Delta E=0.00 / & \left(\nu_{\mathrm{i}}=19.5 i \mathrm{~cm}^{-1}\right) \\
\mu=5.16) & (\Delta G=8.37 / \Delta E=8.98 / \\
& \mu=7.54)
\end{array}
$$

$\mathbf{G}^{* \mathrm{t}} \cdot \mathbf{C}^{*}(\mathrm{rH}) \leftrightarrow \mathbf{G}^{* \mathrm{t}} \cdot \mathbf{C}^{*}\left(\mathbf{w}_{\mathrm{WC} / \mathrm{H}}\right)$ \{rotation around the upper $\mathrm{O6H} \cdots \mathrm{O} 2 \mathrm{H}-\mathrm{bond}$ \}
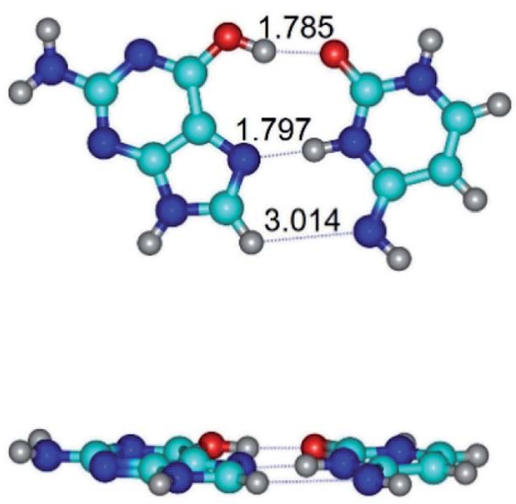

$$
\begin{gathered}
\mathrm{G}^{* \mathrm{t}} \cdot \mathrm{C}^{*}(\mathrm{rH}) \\
(\Delta G=0.00 / \Delta E=0.00 / \\
\mu=5.43)
\end{gathered}
$$
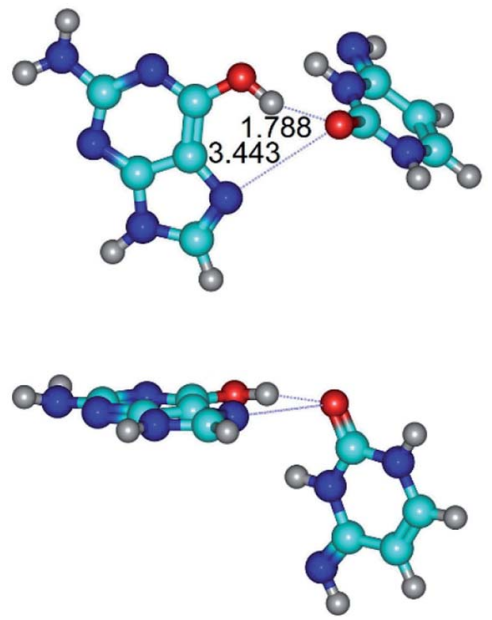

$$
\begin{gathered}
\mathrm{TS}_{\mathrm{G}^{*} \mathrm{t} \cdot \mathrm{C}^{*}(\mathrm{rH})} \leftrightarrow \mathrm{G}^{*} \cdot \mathrm{C}^{*}\left(\mathrm{w}_{\mathrm{WC} / \mathrm{H}}\right) \\
\left(\nu_{\mathrm{i}}=12.0 i \mathrm{~cm}^{-1}\right) \\
(\Delta G=8.79 / \Delta E=9.31 / \\
\mu=6.73)
\end{gathered}
$$

$\mathrm{G}^{* \mathrm{t}} \cdot \mathrm{C}^{*} \mathrm{O} 2_{2}\left(\mathbf{w}_{\mathrm{H}}\right)_{\uparrow} \leftrightarrow \mathrm{G}^{* \mathrm{t}} \cdot \mathrm{C}^{*} \mathrm{O} 2\left(\mathrm{rw}_{\mathrm{H}}\right)_{\downarrow}$ \{rotation around the lower $\mathrm{O} 2 \mathrm{H} \cdots \mathrm{N} 7 \mathrm{H}$-bond

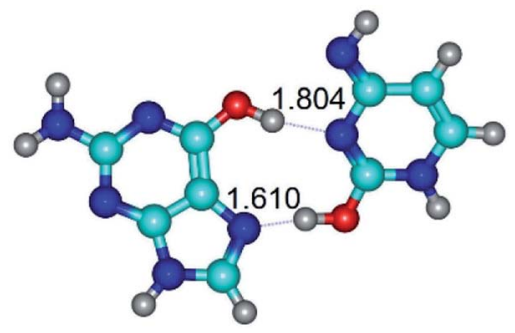

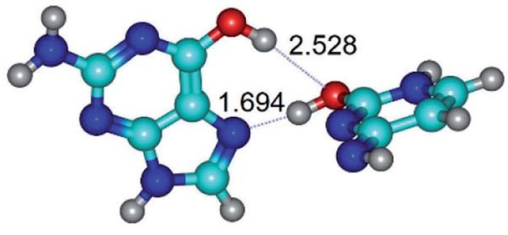

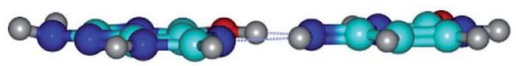

$$
\begin{gathered}
\mathrm{G}^{* \mathrm{t}} \cdot \mathrm{C}^{*}\left(\mathrm{rw}_{\mathrm{H}}\right) \\
(\Delta G=5.28 / \Delta E=7.08 / \mu=7.71)
\end{gathered}
$$
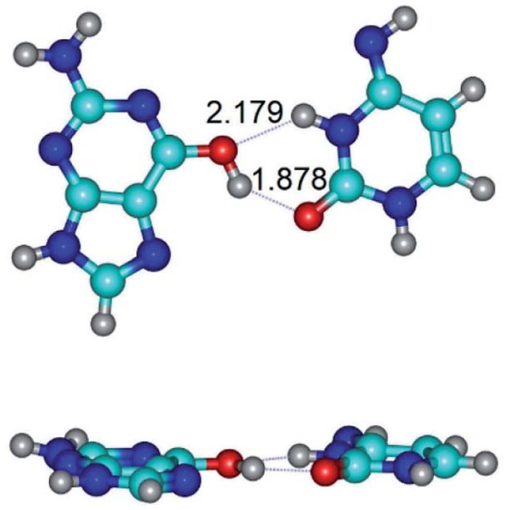

$$
\begin{gathered}
\mathrm{G}^{* \mathrm{t}} \cdot \mathrm{C}^{*}\left(\mathrm{w}_{\mathrm{WC} / \mathrm{H}}\right) \\
(\Delta G=7.05 / \Delta E=9.12 / \mu=4.12)
\end{gathered}
$$

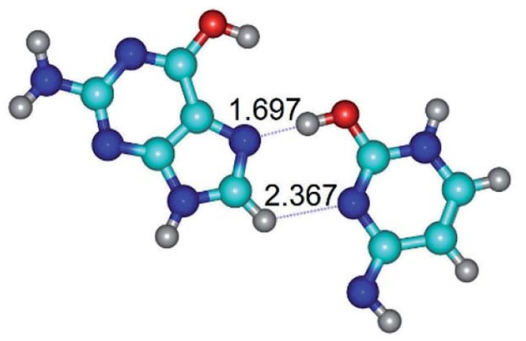




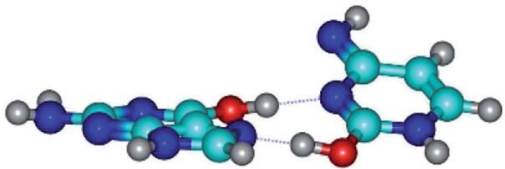

$\mathrm{G}^{* \mathrm{t}} \cdot \mathrm{C}^{*} \mathrm{O} 2_{2}\left(\mathrm{w}_{\mathrm{H}}\right)_{\uparrow}(\Delta G=0.00 /$

$\Delta E=0.00 / \mu=6.70)$

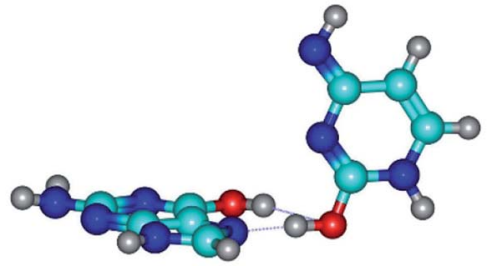

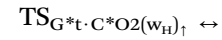

$\mathrm{G}^{*} \mathrm{t} \cdot \mathrm{C} * \mathrm{O} 2\left(\mathrm{rw}_{\mathrm{H}}\right)$

$\left(\nu_{\mathrm{i}}=48.0 i \mathrm{~cm}^{-1}\right)$

$(\Delta G=8.53 / \Delta E=9.22 /$

$\mu=4.25)$

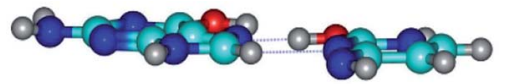

$\mathrm{G}^{* \mathrm{t}} \cdot \mathrm{C}^{*} \mathrm{O} 2\left(\mathrm{rw}_{\mathrm{H}}\right) \downarrow$

$(\Delta G=3.93 / \Delta E=4.69 / \mu=2.36)$
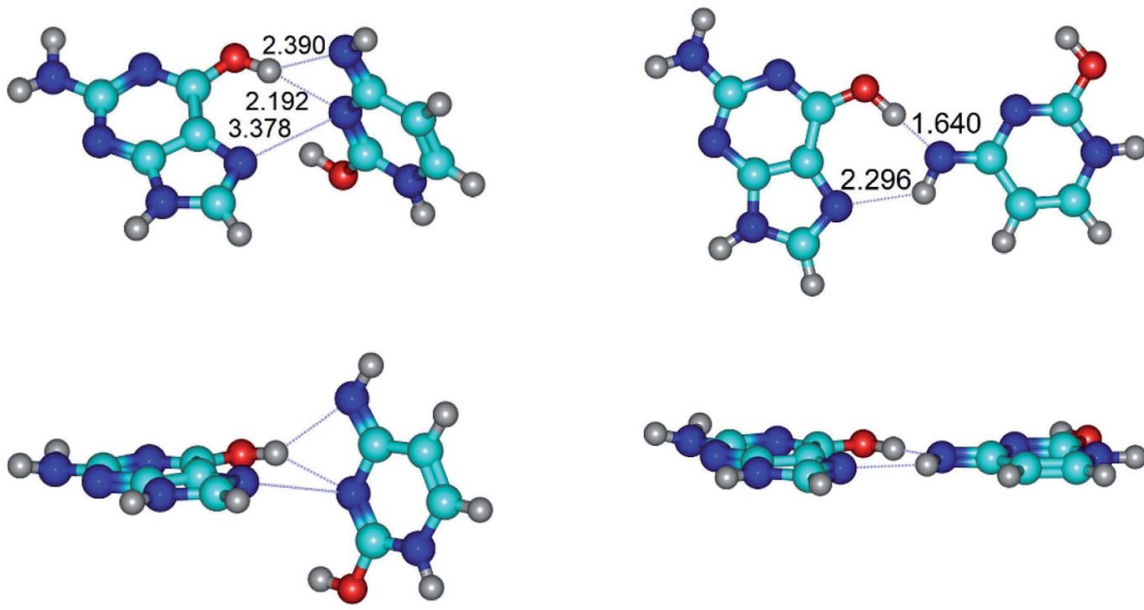

$\mathrm{G}^{* \mathrm{t}} \cdot \mathrm{C}^{*} \mathrm{O} 2\left(\mathrm{w}_{\mathrm{H}}\right)_{\uparrow}$

$(\Delta G=0.00 / \Delta E=0.00 /$ $\mu=6.70)$

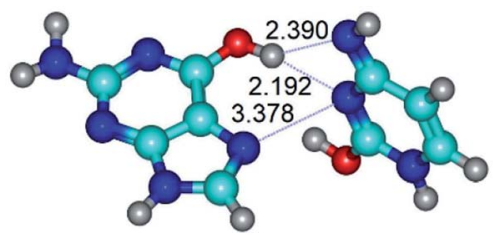

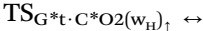

$\mathrm{G}^{* \mathrm{t}} \cdot \mathrm{C}^{*} \mathrm{O} 2\left(\mathrm{rw}_{\mathrm{H}}\right)$

$\left(\nu_{\mathrm{i}}=104.7 i \mathrm{~cm}^{-1}\right)$

$(\Delta G=9.87 / \Delta E=11.67)$

$\mu=7.58)$

$\mathbf{G}^{* \mathrm{t}} \cdot \mathrm{C}^{*} \mathrm{O}_{2}\left(\mathbf{w}_{\mathrm{H}}\right)_{\uparrow} \leftrightarrow \mathbf{G}^{* \mathrm{t}} \cdot \mathrm{C}^{*} \mathrm{O} 2_{2}\left(\mathrm{rw}_{\mathrm{WC} / \mathrm{H}}\right)\{$ rotation around the upper O6H $\cdots \mathrm{N} 3 \mathrm{H}-$ bond $\}$

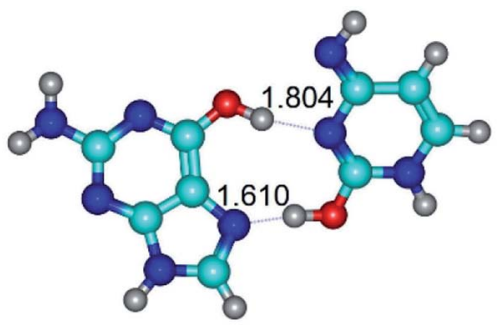

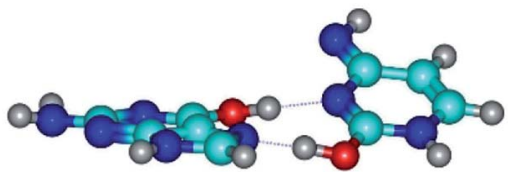

$\mathrm{G}^{* \mathrm{t}} \cdot \mathrm{C}^{*} \mathrm{O} 2\left(\mathrm{w}_{\mathrm{H}}\right)_{\uparrow}$

$(\Delta G=0.00 / \Delta E=0.00 /$ $\mu=6.70)$
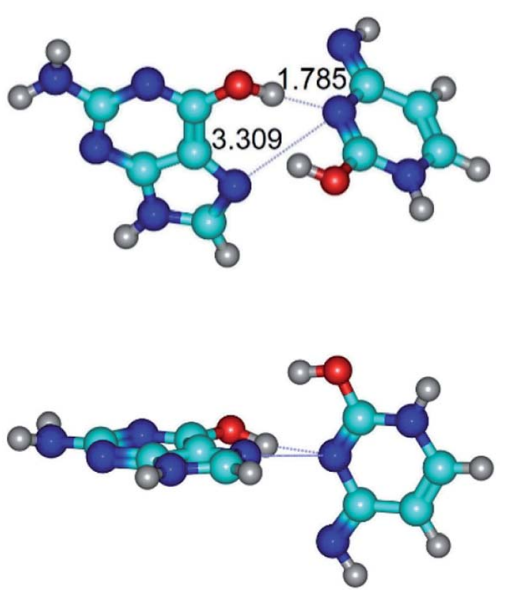

$$
\begin{gathered}
\mathrm{TS}_{\mathrm{G}^{*} \cdot \mathrm{C} \cdot \mathrm{O} 2\left(\mathrm{w}_{\mathrm{H}}\right)_{\uparrow} \leftrightarrow} \\
\mathrm{G}^{*} \cdot \mathrm{C}^{*} \mathrm{O} 2\left(\mathrm{rw}_{\mathrm{wC} / \mathrm{H}}\right) \\
\left(\nu_{\mathrm{i}}=42.0 i \mathrm{~cm}{ }^{-1}\right) \\
(\Delta G=9.67 / \Delta E=9.96 / \\
\mu=7.55)
\end{gathered}
$$
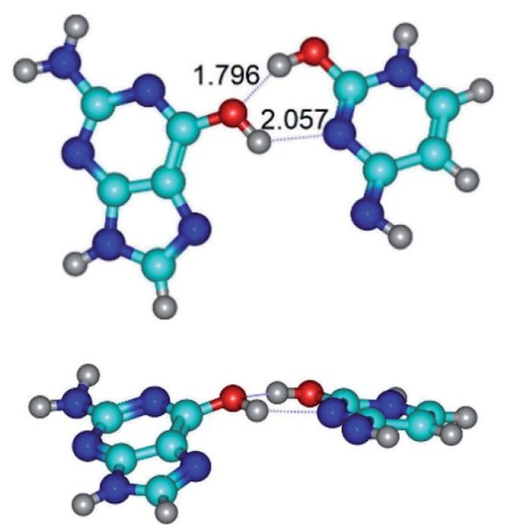

$\mathrm{G}^{* \mathrm{t}} \cdot \mathrm{C}^{*}{ }_{\mathrm{O} 2}\left(\mathrm{rw}_{\mathrm{WC} / \mathrm{H}}\right)$

$(\Delta G=8.47 / \Delta E=9.73 / \mu=2.47)$ 
Table 1 (Contd.)

$\mathrm{G}^{* \mathrm{t}} \cdot \mathrm{C}^{* \mathrm{t}}{ }_{\mathrm{O} 2}\left(\mathrm{w}_{\mathrm{H}}\right)_{\uparrow} \leftrightarrow \mathrm{G}^{* \mathrm{t}} \cdot \mathrm{C}^{* \mathrm{t}}{ }_{\mathrm{O} 2}\left(\mathrm{rw}_{\mathrm{H}}\right)_{\uparrow}$ \{rotation around the middle O6H $\cdots \mathrm{N} 3 \mathrm{H}$-bond
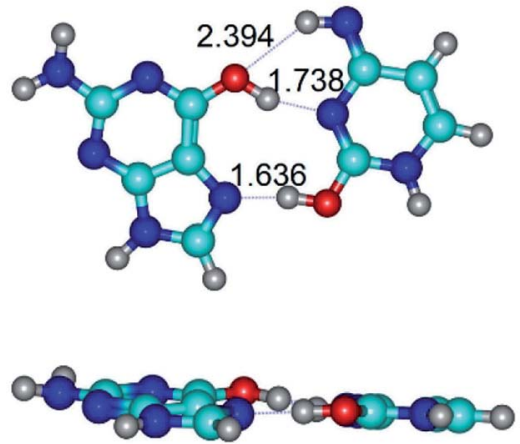

$$
\begin{gathered}
\mathrm{G}^{* \mathrm{t}} \cdot \mathrm{C}^{* \mathrm{t}} \mathrm{O2}\left(\mathrm{w}_{\mathrm{H}}\right)_{\uparrow} \\
(\Delta G=0.00 / \Delta E=0.00 / \\
\mu=7.97)
\end{gathered}
$$
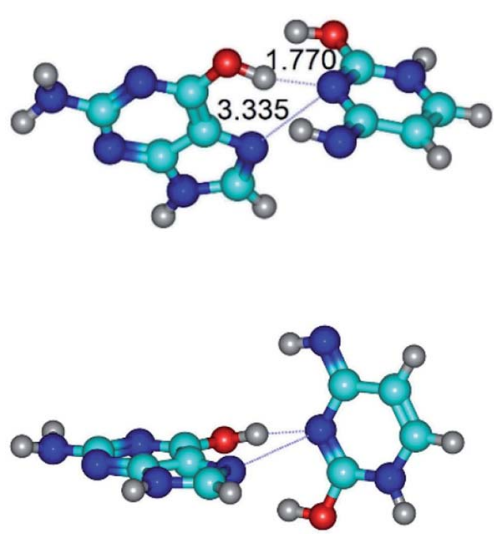

$$
\begin{gathered}
\mathrm{TS}_{\mathrm{G}^{*} \cdot \mathrm{C}^{*} \mathrm{tO} 2\left(\mathrm{w}_{\mathrm{H}}\right)_{\uparrow} \leftrightarrow} \\
\mathrm{G}^{*} \cdot \mathrm{C}^{*} \mathrm{tO}^{2}\left(\mathrm{rw}_{\mathrm{H}}\right)_{\uparrow} \\
\left(\nu_{\mathrm{i}}=32.2 i \mathrm{~cm}^{-1}\right) \\
(\Delta G=10.62 / \Delta E=10.82 / \\
\mu=6.28)
\end{gathered}
$$

$\mathbf{G}^{* \mathrm{t}} \cdot \mathbf{C}^{* \mathrm{t}}{ }_{\mathrm{O} 2}\left(\mathbf{W}_{\mathbf{H}}\right)_{\uparrow} \leftrightarrow \mathbf{G}^{* \mathrm{t}} \cdot \mathbf{C}^{* \mathrm{t}}{ }_{\mathrm{O} 2}\left(\mathrm{rw}_{\mathbf{H}}\right)_{\downarrow}$ \{rotation around the lower $\mathrm{O} 2 \mathrm{H} \cdots \mathrm{N} 7 \mathrm{H}$-bond
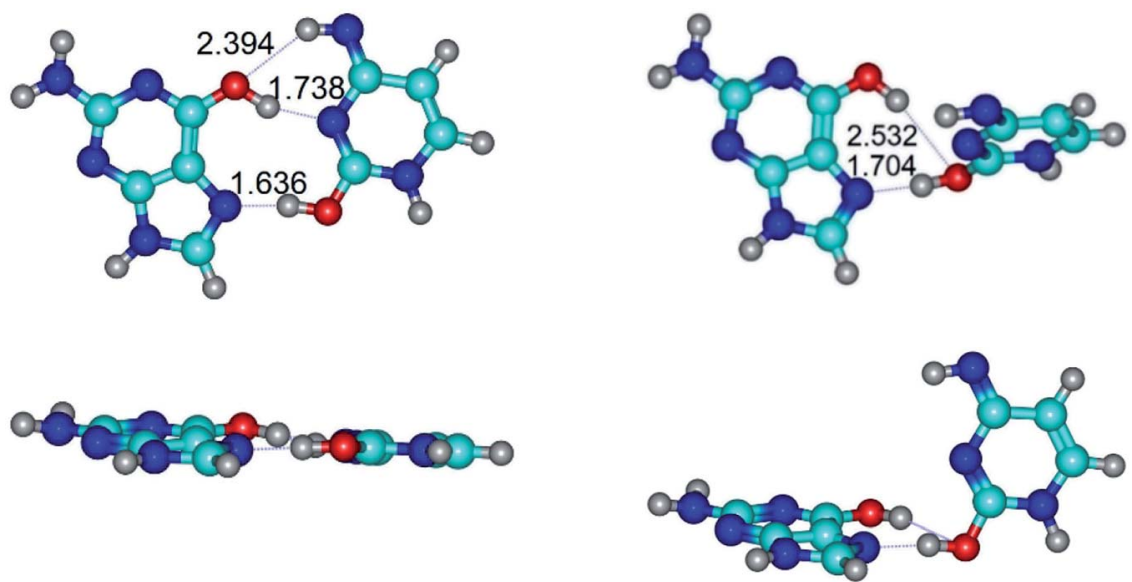

$$
\begin{gathered}
\mathrm{G}^{* \mathrm{t}} \cdot \mathrm{C}^{* \mathrm{t}} \mathrm{O} 2\left(\mathrm{w}_{\mathrm{H}}\right) \uparrow \\
(\Delta G=0.00 / \Delta E=0.00 / \\
\mu=7.97)
\end{gathered}
$$

$$
\begin{gathered}
\mathrm{TS}_{\mathrm{G}^{*} \cdot \mathrm{C}^{*} \mathrm{tO} 2\left(\mathrm{w}_{\mathrm{H}}\right)_{\uparrow} \leftrightarrow} \\
\mathrm{G}^{*} \cdot \mathrm{C}^{*} \mathrm{tO} 2\left(\mathrm{rw}_{\mathrm{H}}\right)_{\downarrow} \\
\left.\left(\nu_{\mathrm{i}}=45.2 i \mathrm{~cm}\right)^{-1}\right) \\
(\Delta G=9.15 / \Delta E=9.55 / \\
\mu=5.92)
\end{gathered}
$$

$\mathrm{G}^{* \mathrm{t}} \cdot \mathrm{C}^{* \mathrm{t}}{ }_{\mathrm{O} 2}\left(\mathrm{rw}_{\mathrm{H}}\right)_{\downarrow} \leftrightarrow \mathbf{G}^{* \mathrm{t}} \cdot \mathrm{C}^{* \mathrm{t}}{ }_{\mathrm{O} 2}\left(\mathbf{w}_{\mathrm{H}}\right)_{\downarrow}$ \{rotation around the lower $\mathbf{C 8 H} \cdots \mathbf{N} 3 \mathrm{H}-$ bond $\}$
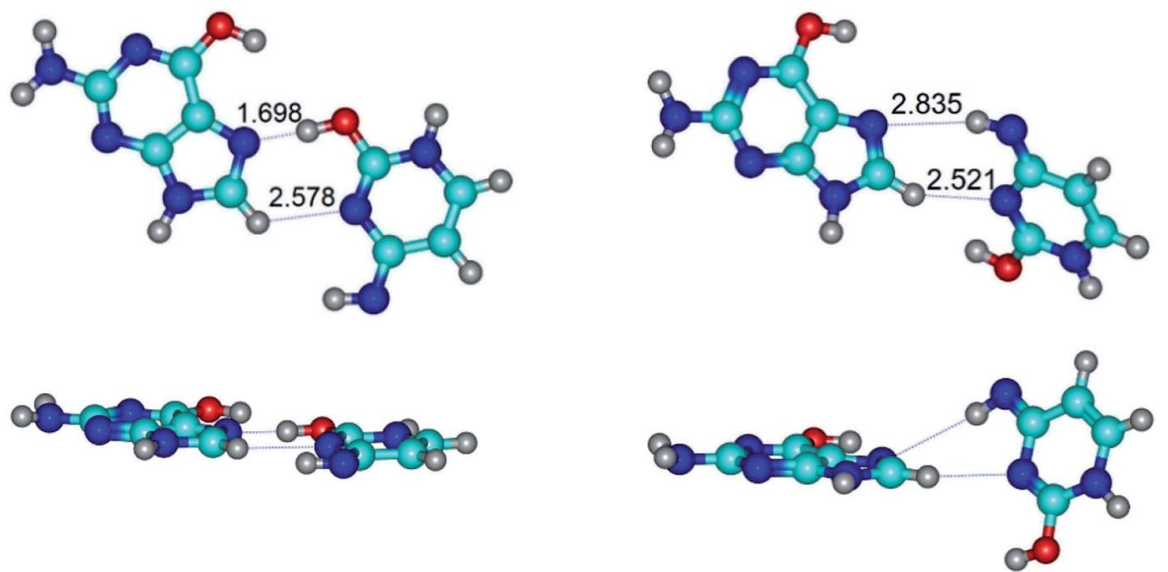
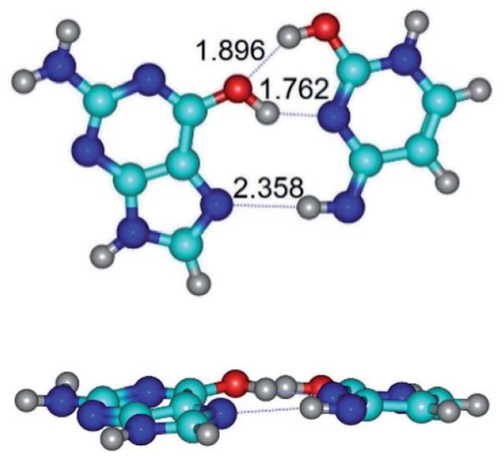

$$
\begin{gathered}
\mathrm{G}^{* \mathrm{t}} \cdot \mathrm{C}^{* \mathrm{t}}{ }_{\mathrm{O} 2}\left(\mathrm{rw}_{\mathrm{H}}\right)_{\uparrow} \\
(\Delta G=6.55 / \Delta E=6.58 / \mu=1.39)
\end{gathered}
$$
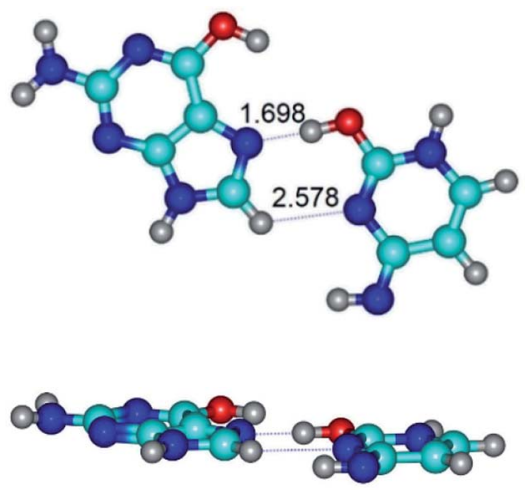

$$
\begin{gathered}
\mathrm{G}^{* \mathrm{t}} \cdot \mathrm{C}^{* \mathrm{t}}{ }_{\mathrm{O} 2}\left(\mathrm{rw}_{\mathrm{H}}\right)_{\downarrow} \\
(\Delta G=6.31 / \Delta E=7.84 / \mu=2.39)
\end{gathered}
$$
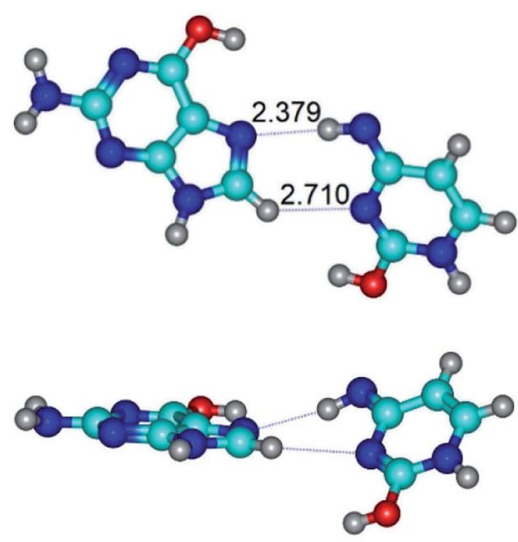
Table 1 (Contd.)

$$
\begin{gathered}
\mathrm{G}^{* \mathrm{t}} \cdot \mathrm{C}^{* \mathrm{t}}{ }_{\mathrm{O} 2}\left(\mathrm{rw}_{\mathrm{H}}\right)_{\downarrow} \\
(\Delta G=0.00 / \Delta E=0.00 / \\
\mu=2.39)
\end{gathered}
$$
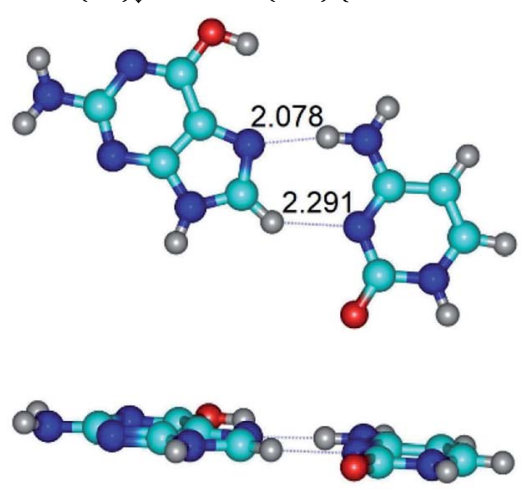

$$
\begin{gathered}
\mathrm{G}^{* \mathrm{t}} \cdot \mathrm{C}\left(\mathrm{w}_{\mathrm{H}}\right)_{\downarrow} \\
(\Delta G=0.00 / \Delta E=0.00 / \\
\mu=4.66)
\end{gathered}
$$

$\mathbf{G}^{* \mathrm{t}} \cdot \mathbf{C}\left(\mathbf{r w}_{\mathbf{H}}\right)_{\uparrow} \leftrightarrow \mathbf{G}^{* \mathrm{t}} \cdot \mathbf{C}\left(\mathbf{w}_{\mathbf{H}}\right)_{\downarrow}$ \{rotation around the lower $\mathbf{N} 4 \mathbf{H}^{\cdots} \mathrm{N}$ 7 H-bond
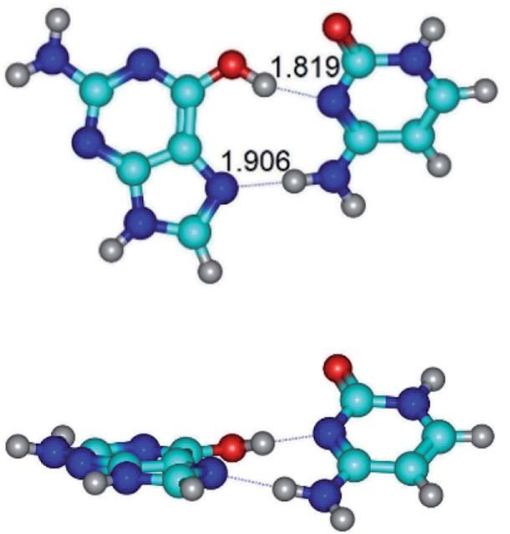

$\mathrm{G}^{* \mathrm{t}} \cdot \mathrm{C}\left(\mathrm{rw}_{\mathrm{H}}\right)_{\uparrow}$

$(\Delta G=0.00 / \Delta E=0.00$ $\mu=8.25)$
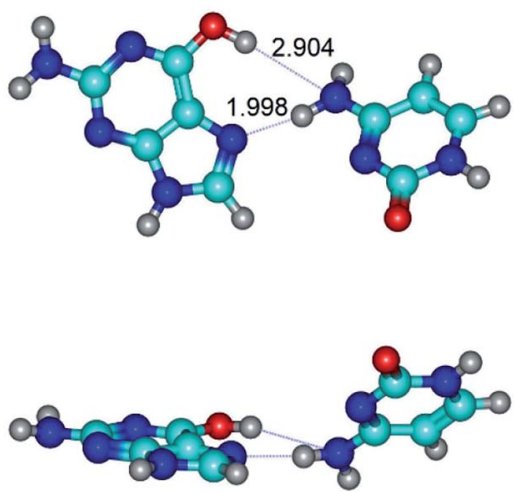

$\mathrm{TS}_{\mathrm{G}} *_{t} \cdot \mathrm{C}\left(\mathrm{w}_{\mathrm{H}}\right)_{\downarrow} \leftrightarrow \mathrm{G}^{*} \mathrm{t} \cdot \mathrm{C}\left(\mathrm{rw}_{\mathrm{H}}\right)$
$\left(\nu_{\mathrm{i}}=26.7 i \mathrm{~cm}^{-1}\right)$

$(\Delta G=4.74 / \Delta E=3.84 /$

$$
\mu=3.41)
$$
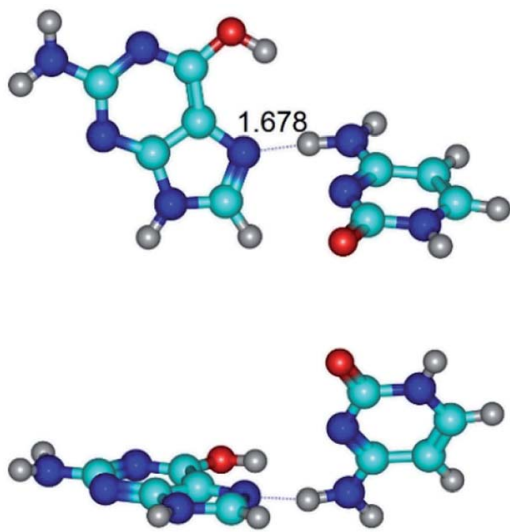

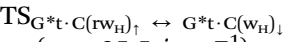

$\left(\nu_{\mathrm{i}}=25.5 i \mathrm{~cm}^{-1}\right)$

$(\Delta G=5.72 / \Delta E=3.92 /$

$$
\mu=4.50)
$$
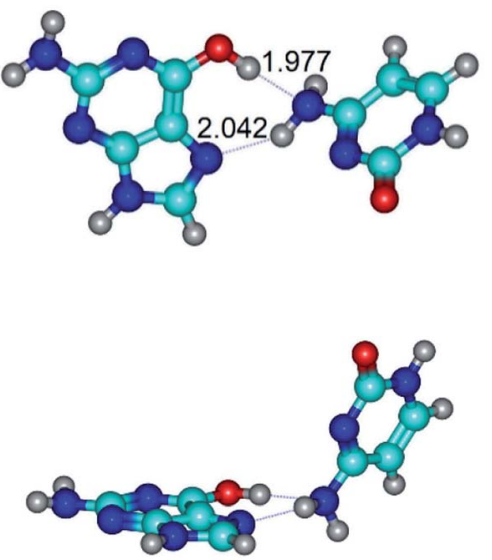

$\mathrm{G}^{* \mathrm{t}} \cdot \mathrm{C}\left(\mathrm{rw}_{\mathrm{H}}\right)$

$(\Delta G=2.45 / \Delta E=1.23 / \mu=4.29)$
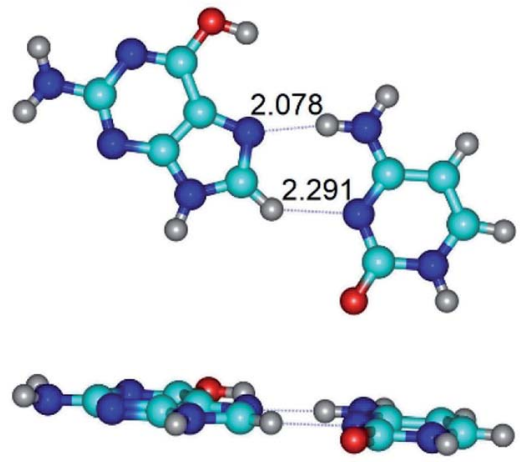

$$
\begin{gathered}
\mathrm{G}^{* \mathrm{t}} \cdot \mathrm{C}\left(\mathrm{w}_{\mathrm{H}}\right)_{\downarrow} \\
(\Delta G=2.73 / \Delta E=4.24 / \mu=4.66)
\end{gathered}
$$

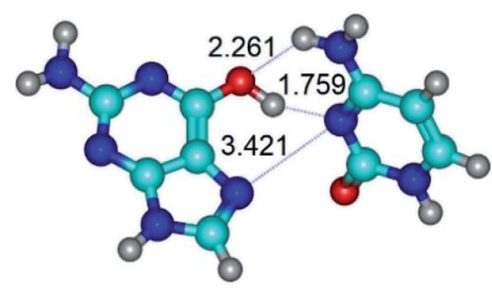




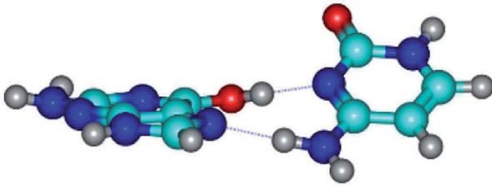

$\mathrm{G}^{* \mathrm{t}} \cdot \mathrm{C}\left(\mathrm{rw}_{\mathrm{H}}\right)_{\uparrow}$

$(\Delta G=0.00 / \Delta E=0.00$

$\mu=8.25)$

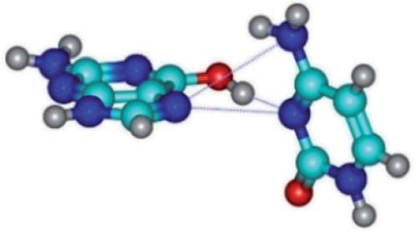

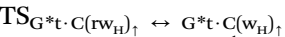
$\left(\nu_{\mathrm{i}}=32.2 i \mathrm{~cm}^{-1}\right)$

$(\Delta G=3.67 / \Delta E=3.64$ $\mu=8.84)$

$\mathbf{G}^{*}{ }_{\mathrm{N} 7} \cdot \mathbf{C}^{*}\left(\mathrm{rw}_{\mathrm{H}}\right)_{\uparrow} \leftrightarrow \mathbf{G}^{*}{ }_{\mathrm{N} 7} \cdot \mathbf{C}^{*}\left(\mathbf{w}_{\mathbf{H}}\right)_{\uparrow}$ \{rotation around the upper N3H $\cdots$ O6 H-bond
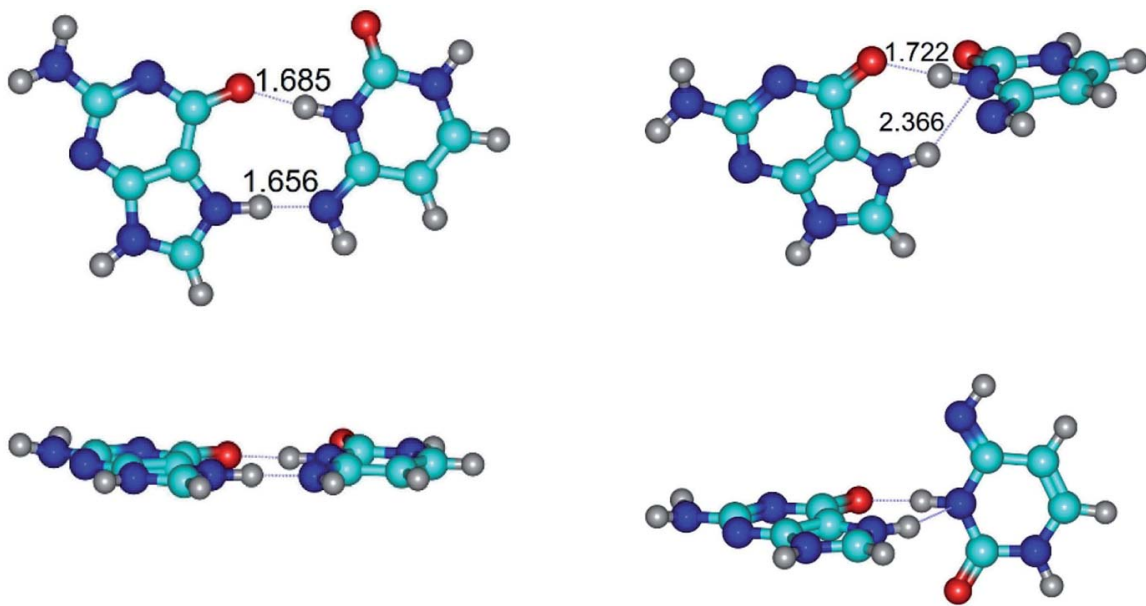

$$
\begin{gathered}
\mathrm{G}^{*}{ }_{\mathrm{N} 7} \cdot \mathrm{C}^{*}\left(\mathrm{rw}_{\mathrm{H}}\right)_{\uparrow} \\
(\Delta G=0.00 / \Delta E=0.00 / \\
\mu=10.28)
\end{gathered}
$$

$$
\begin{gathered}
\mathrm{TS}_{\mathrm{G}^{*} \mathrm{~N} 7 \cdot \mathrm{C}^{*}\left(\mathrm{rw}_{\mathrm{H}}\right)_{\uparrow} \leftrightarrow} \\
\mathrm{G}^{*} \mathrm{~N} 7 \cdot \mathrm{C}^{*}\left(\mathrm{w}_{\mathrm{H}}\right)_{\uparrow} \\
\left(\nu_{\mathrm{i}}=34.7 i \mathrm{~cm}^{-1}\right) \\
(\Delta G=13.36 / \Delta E=13.06 / \\
\mu=9.49)
\end{gathered}
$$

$\mathbf{G}^{*}{ }_{\mathbf{N} 7} \cdot \mathbf{C}^{*}\left(\mathbf{r w}_{\mathbf{H}}\right)_{\uparrow} \leftrightarrow \mathbf{G}^{*}{ }_{\mathbf{N} 7} \cdot \mathbf{C}^{*}\left(\mathbf{w}_{\mathbf{H}}\right)\{$ rotation around the lower $\mathbf{N 7 H} \cdots \mathbf{N} 4 \mathbf{H}-$-bond $\}$
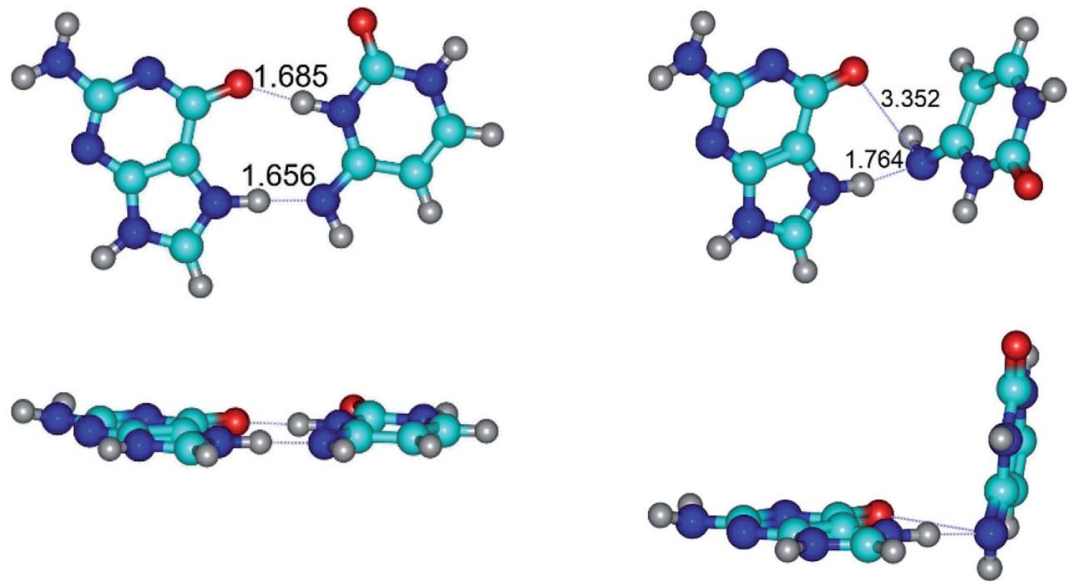

$$
\begin{gathered}
\mathrm{G}^{*}{ }_{\mathrm{N} 7} \cdot \mathrm{C}^{*}\left(\mathrm{rw}_{\mathrm{H}}\right)_{\uparrow} \\
(\Delta G=0.00 / \Delta E=0.00 / \\
\mu=10.28)
\end{gathered}
$$

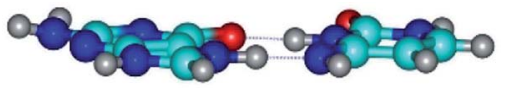

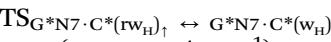

$$
\begin{aligned}
& \left(\nu_{\mathrm{i}}=19.5 i \mathrm{~cm}^{-1}\right) \\
& (\Delta G=8.70 / \Delta E=7.53 / \\
& \mu=4.58)
\end{aligned}
$$

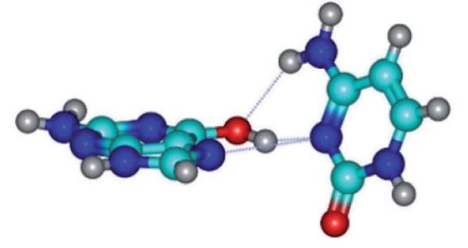

$\mathrm{G}^{* \mathrm{t}} \cdot \mathrm{C}\left(\mathrm{w}_{\mathrm{H}}\right)_{\uparrow}$

$(\Delta G=2.00 / \Delta E=3.34 / \mu=7.91)$
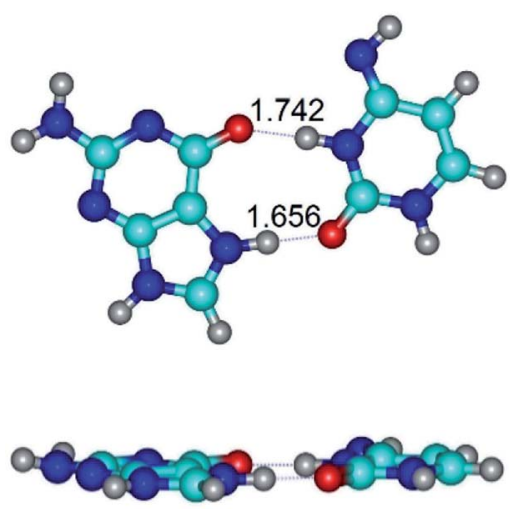

$$
\begin{gathered}
\mathrm{G}^{*}{ }_{\mathrm{N} 7} \cdot \mathrm{C}^{*}\left(\mathrm{w}_{\mathrm{H}}\right)_{\uparrow} \\
(\Delta G=3.25 / \Delta E=3.12 / \mu=9.46)
\end{gathered}
$$
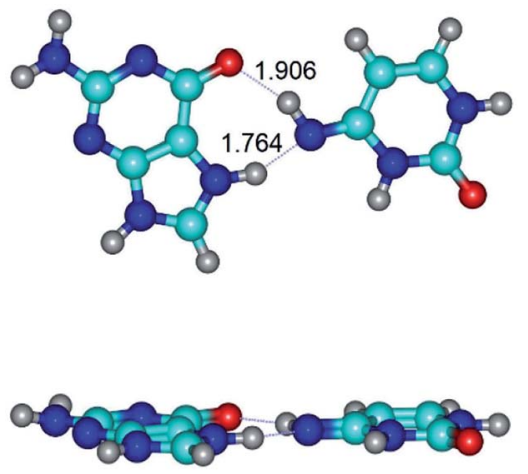

$\mathrm{G}^{*}{ }_{\mathrm{N} 7} \cdot \mathrm{C}^{*}\left(\mathrm{w}_{\mathrm{H}}\right)$

$(\Delta G=4.62 / \Delta E=4.71 / \mu=4.42)$ 
Table 1 (Contd.)

$\mathrm{G}^{*}{ }_{\mathrm{N} 7} \cdot \mathrm{C}^{* \mathrm{t}}\left(\mathbf{w}_{\mathrm{H}}\right)_{\uparrow} \leftrightarrow \mathrm{G}^{*}{ }_{\mathrm{N} 7} \cdot \mathrm{C}^{* \mathrm{t}}\left(\mathbf{r w}_{\mathrm{H}}\right)_{\uparrow}\{$ rotation around the middle $\mathrm{N} 3 \mathrm{H} \cdots \mathrm{O}$. H-bond
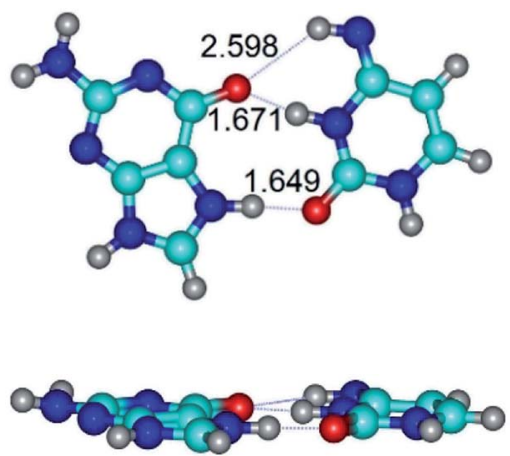

$$
\begin{gathered}
\mathrm{G}^{*}{ }_{\mathrm{N} 7} \cdot \mathrm{C}^{* \mathrm{t}}\left(\mathrm{W}_{\mathrm{H}}\right)_{\uparrow} \\
(\Delta G=0.00 / \Delta E=0.00 / \\
\mu=9.52)
\end{gathered}
$$
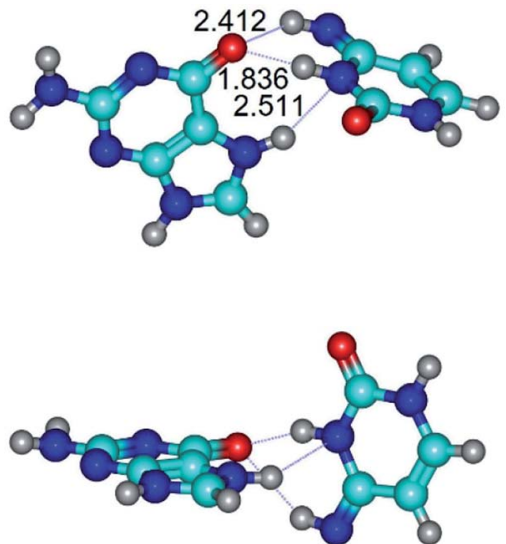

$$
\begin{gathered}
\mathrm{TS}_{\mathrm{G} * \mathrm{~N} 7 \cdot \mathrm{C}^{*} \mathrm{t}\left(\mathrm{w}_{\mathrm{H}}\right)_{\uparrow} \leftrightarrow} \\
\mathrm{G}^{*} \mathrm{~N} 7 \cdot \mathrm{C}^{*} \mathrm{t}\left(\mathrm{rw}_{\mathrm{H}}\right)_{\uparrow} \\
\left(\nu_{\mathrm{i}}=46.0 i \mathrm{~cm}^{-1}\right) \\
(\Delta G=12.63 / \Delta E=12.92 / \\
\mu=9.89)
\end{gathered}
$$

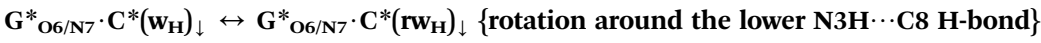
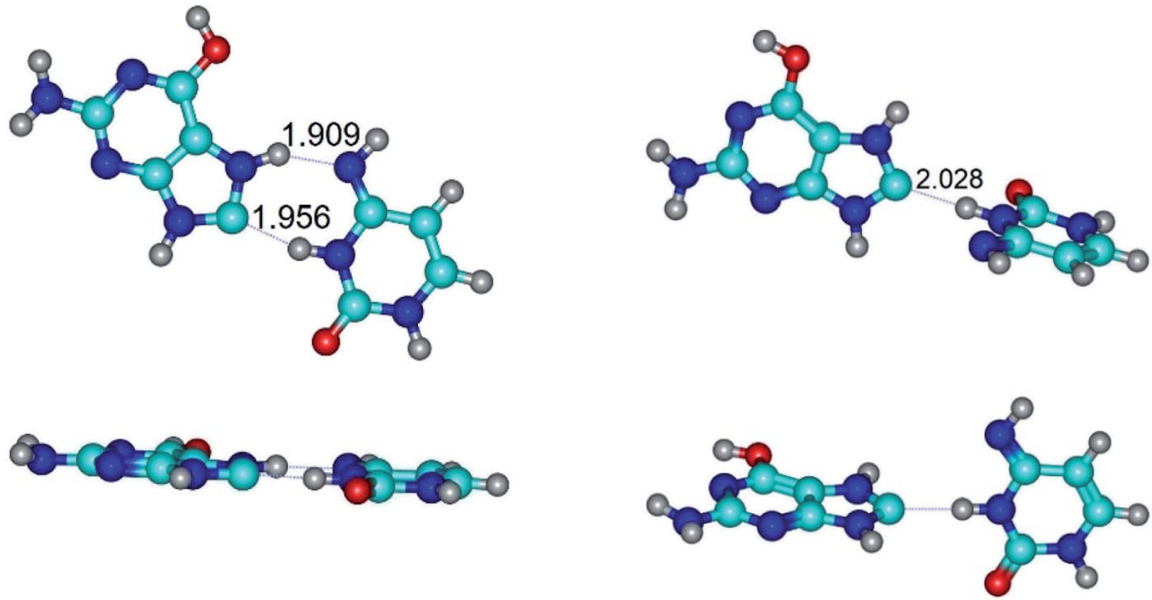

$$
\begin{gathered}
\mathrm{G}^{*}{ }_{\mathrm{O} / \mathrm{N} \mathrm{N}} \cdot \mathrm{C}^{*}\left(\mathrm{w}_{\mathrm{H}}\right)_{\downarrow} \\
(\Delta G=0.00 / \Delta E=0.00 / \\
\mu=3.49)
\end{gathered}
$$

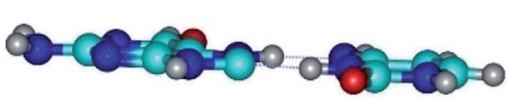

$\mathbf{G}^{*}{ }_{06 / \mathrm{N} 7} \cdot \mathbf{C}^{*}\left(\mathbf{w}_{\mathbf{H}}\right)_{\downarrow} \leftrightarrow \mathbf{G}^{*}{ }_{\mathrm{O} / \mathrm{N} 7} \cdot \mathbf{C}^{*}\left(\mathrm{rw}_{\mathbf{H}}\right)_{\uparrow}$ \{rotation around the upper N7H $\cdots \mathbf{N} 4$ H-bond

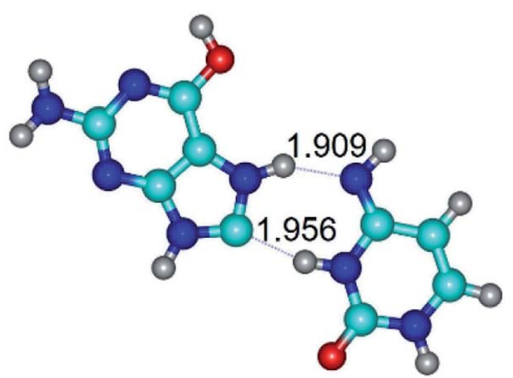

$\mathrm{TS}_{\mathrm{G}} * \mathrm{O} 6 / \mathrm{N} 7 \cdot \mathrm{C}^{*}\left(\mathrm{w}_{\mathrm{H}}\right)_{\downarrow} \leftrightarrow \mathrm{G}^{*} \mathrm{O} 6 /$

$$
\begin{gathered}
\mathrm{N} 7 \cdot \mathrm{C}^{*}\left(\mathrm{rw}_{\mathrm{H}}\right)_{\downarrow} \\
\left(\nu_{\mathrm{i}}=20.2 i \mathrm{~cm}^{-1}\right) \\
(\Delta G=8.82 / \Delta E=9.66 \\
\mu=1.52)
\end{gathered}
$$

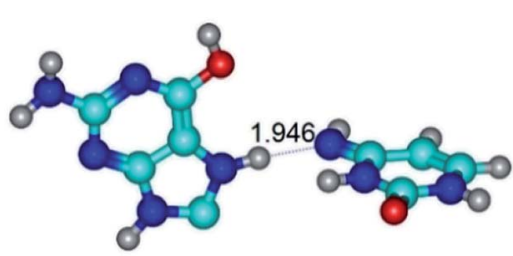

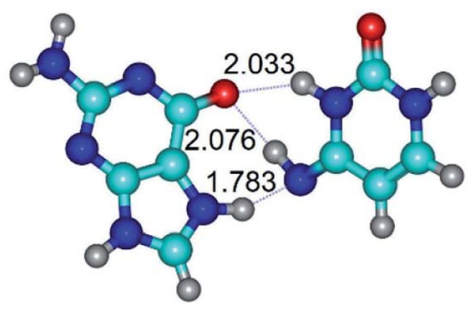

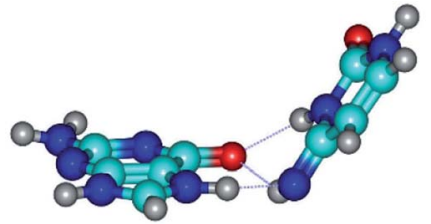

$$
\begin{gathered}
\mathrm{G}^{*}{ }_{\mathrm{N} 7} \cdot \mathrm{C}^{* \mathrm{t}}\left(\mathrm{rw}_{\mathrm{H}}\right)_{\uparrow} \\
(\Delta G=6.43 / \Delta E=6.23 / \mu=9.80)
\end{gathered}
$$
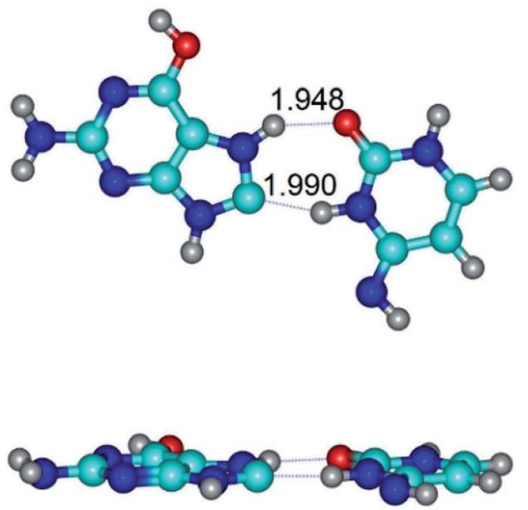

$\mathrm{G}^{*}{ }_{\mathrm{O} 6 / \mathrm{N} 7} \cdot \mathrm{C}^{*}\left(\mathrm{rw}_{\mathrm{H}}\right)_{\downarrow}$

$(\Delta G=2.29 / \Delta E=2.42 / \mu=2.49)$

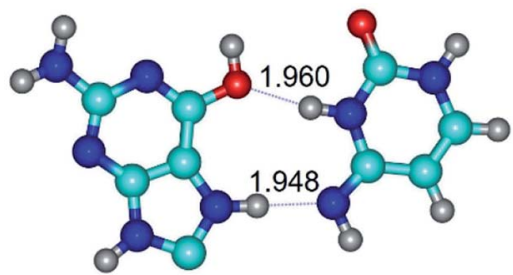




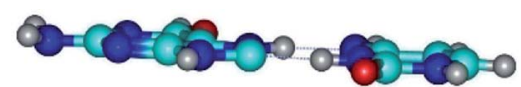

$\mathrm{G}^{*}{ }_{\mathrm{O} 6 / \mathrm{N} 7} \cdot \mathrm{C}^{*}\left(\mathrm{w}_{\mathrm{H}}\right)_{\downarrow}$

$(\Delta G=0.00 / \Delta E=0.00 /$

$\mu=3.49)$

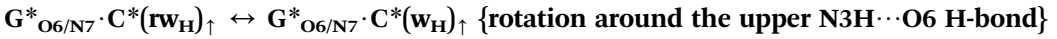
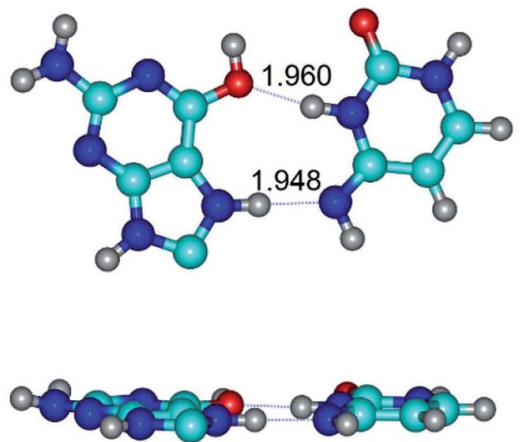

$\mathrm{G}^{*} \mathrm{O} 6 / \mathrm{N} 7 \cdot \mathrm{C}^{*}\left(\mathrm{rw}_{\mathrm{H}}\right)_{\uparrow}$

$(\Delta G=0.00 / \Delta E=0.00 /$

$\mu=5.21)$
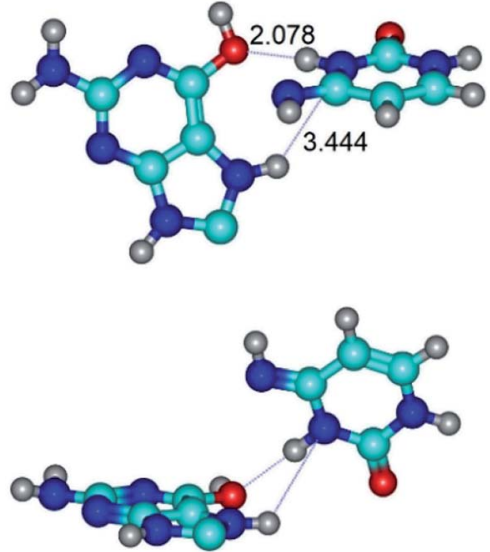

$\mathrm{TS}_{\mathrm{G}^{*}} \mathrm{O} / \mathrm{N} 7 \cdot \mathrm{C}^{*}\left(\mathrm{rw}_{\mathrm{H}}\right)_{\uparrow} \leftrightarrow \mathrm{G}^{*} \mathrm{O} 6$

N7. $\mathrm{C}^{*}\left(\mathrm{w}_{\mathrm{H}}\right)$,

$\left(\nu_{\mathrm{i}}=16.3 i \mathrm{~cm}^{-1}\right)$

$(\Delta G=6.72 / \Delta E=7.98 /$

$\mu=4.79)$

$\mathrm{G}^{*}{ }_{\mathrm{o6} / \mathrm{N} 7} \cdot \mathrm{C}^{*}\left(\mathrm{rw}_{\mathbf{H}}\right)_{\downarrow} \leftrightarrow \mathrm{G}^{*}{ }_{\mathrm{o6} / \mathrm{N} 7} \cdot \mathrm{C}^{*}\left(\mathbf{w}_{\mathbf{H}}\right)_{\uparrow}$ \{rotation around the upper N7H $\cdots \mathrm{O} 2 \mathrm{H}$-bond $\}$
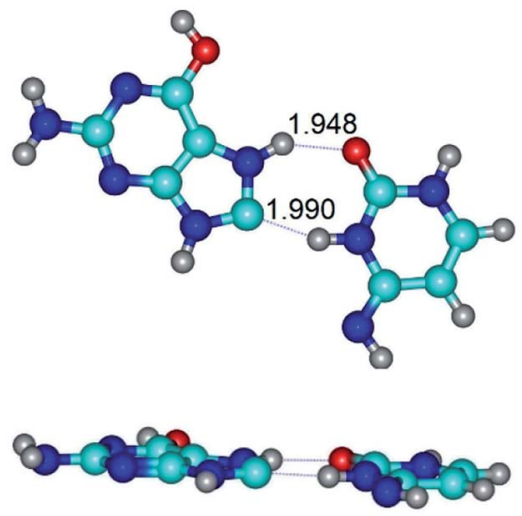

$\mathrm{G}^{*}{ }_{\mathrm{O} 6 / \mathrm{N} 7} \cdot \mathrm{C}^{*}\left(\mathrm{rw}_{\mathrm{H}}\right)_{\downarrow}$

$(\Delta G=0.00 / \Delta E=0.00 /$

$\mu=2.49)$
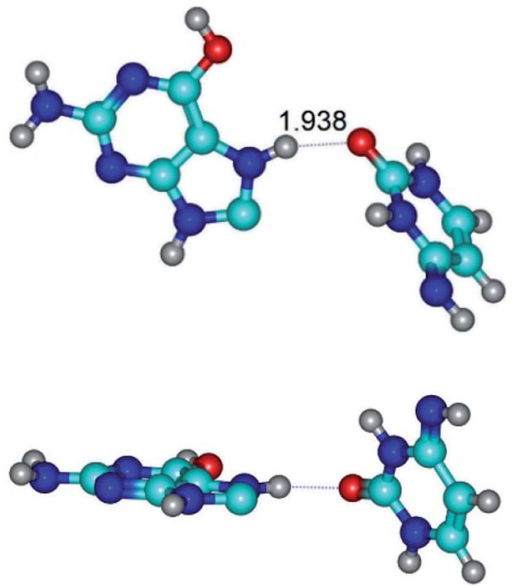

$\mathrm{TS}_{\mathrm{G}} * \mathrm{O} 6 / \mathrm{N} 7 \cdot \mathrm{C} *\left(\mathrm{rw}_{\mathrm{H}}\right)_{\downarrow} \leftrightarrow \mathrm{G} * \mathrm{O} 6 /$

$\mathrm{N} 7 \cdot \mathrm{C}^{*}\left(\mathrm{w}_{\mathrm{H}}\right)_{+}$

$\left(\nu_{\mathrm{i}}=20.6 i \mathrm{~cm}^{-1}\right)$

$(\Delta G=6.75 / \Delta E=7.56 /$

$\mu=3.19)$

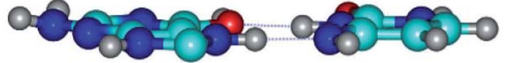

$\mathrm{G}^{*}{ }_{\mathrm{O} 6 / \mathrm{N} 7} \cdot \mathrm{C}^{*}\left(\mathrm{rw}_{\mathrm{H}}\right)_{\uparrow}$

$(\Delta G=4.17 / \Delta E=4.14 / \mu=5.21)$
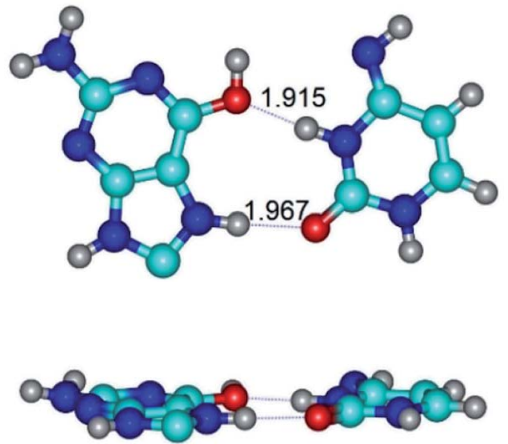

$\mathrm{G}^{*}{ }_{\mathrm{O} / \mathrm{N} 7} \cdot \mathrm{C} *\left(\mathrm{w}_{\mathrm{H}}\right)_{\uparrow}$

$(\Delta G=1.66 / \Delta E=1.97 / \mu=5.68)$
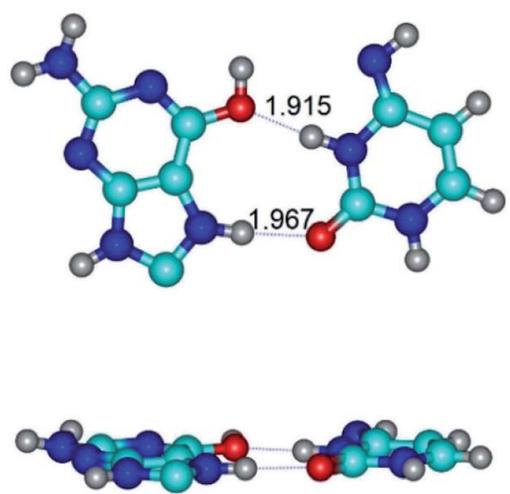

$\mathrm{G}^{*}{ }_{\mathrm{O} 6 / \mathrm{N} 7} \cdot \mathrm{C}^{*}\left(\mathrm{w}_{\mathrm{H}}\right)_{\uparrow}$

$(\Delta G=3.54 / \Delta E=3.68 / \mu=5.68)$ 
Table 1 (Contd.)

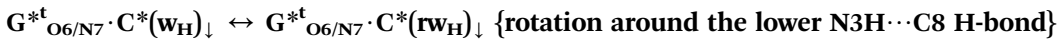
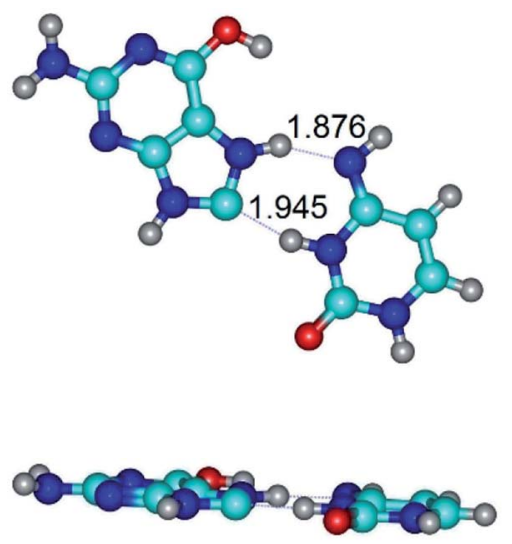

$\mathrm{G}^{* \mathrm{t}} \mathrm{O6/ \textrm {N } 7} \cdot \mathrm{C} *\left(\mathrm{w}_{\mathrm{H}}\right)_{\downarrow}$
$(\Delta G=0.00 / \Delta E=0.00 /$
$\mu=4.81)$
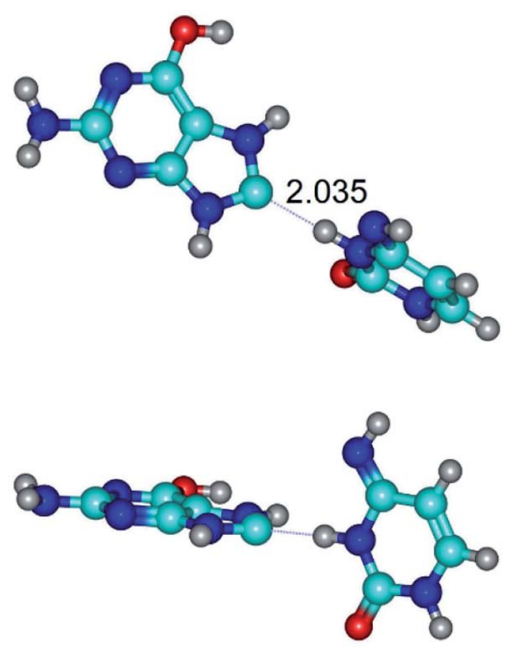

$$
\begin{gathered}
\mathrm{TS}_{\mathrm{G} * \mathrm{tO} 6 / \mathrm{N} 7 \cdot \mathrm{C}^{*}\left(\mathrm{w}_{\mathrm{H}}\right)_{\downarrow} \leftrightarrow \mathrm{G}^{*} \mathrm{tO} 6 /} \\
\mathrm{N} 7 \cdot \mathrm{C} *\left(\mathrm{rw}_{\mathrm{H}}\right)_{\downarrow} \\
\left(\nu_{\mathrm{i}}=18.1 i \mathrm{~cm}^{-1}\right) \\
(\Delta G=10.40 / \Delta E=11.68 / \\
\mu=2.71)
\end{gathered}
$$
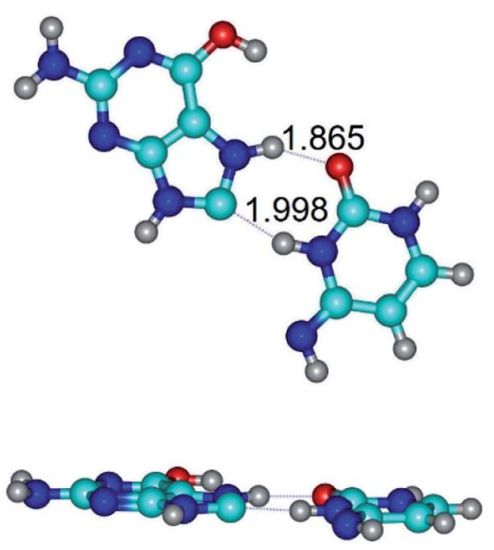

$\mathrm{G}^{* \mathrm{t}}{ }_{\mathrm{O} 6 / \mathrm{N} 7} \cdot \mathrm{C} *\left(\mathrm{rw}_{\mathrm{H}}\right)_{\downarrow}$ $(\Delta G=2.25 / \Delta E=2.28 / \mu=4.41)$ nucleobase pair. This $\mathrm{G} \cdot \mathrm{C}(\mathrm{WC}) \leftrightarrow \mathrm{G} \cdot \mathrm{C}\left(\mathrm{rw}_{\mathrm{WC}}\right)$ conformational transformation occurs by the rotation of the $\mathrm{G}$ and $\mathrm{C}$ bases accordingly each other around the middle intermolecular (G) $\mathrm{N} 1 \mathrm{H} \cdots \mathrm{N} 3(\mathrm{C}) \mathrm{H}$-bond and is accompanied by the rebuilding of the intermolecular H-bonds: (C)N4H $\cdots \mathrm{O} 6(\mathrm{G}),(\mathrm{G}) \mathrm{N} 1 \mathrm{H} \cdots \mathrm{N} 3(\mathrm{C})$, $(\mathrm{G}) \mathrm{N} 2 \mathrm{H} \cdots \mathrm{O} 2(\mathrm{C}) \rightarrow(\mathrm{G}) \mathrm{N} 1 \mathrm{H} \cdots \mathrm{N} 3(\mathrm{C}),(\mathrm{G}) \mathrm{N} 2 \mathrm{H} \cdots \mathrm{N} 3(\mathrm{C}),(\mathrm{C}) \mathrm{N} 4 \mathrm{H} \cdots$ N2(G). Unusually, this transition leads to the changing of the geometry from the Watson-Crick-like to wobble-like, which is significantly non-planar with significantly non-planar $\mathrm{NH}_{2}$ amino group of the $\mathrm{G}$ base. Notably, that during this transition nucleobases do not change their tautomeric status. This obtained data complement the results of the previous works. ${ }^{42,48,49}$

2. It was established that rotation of the $\mathrm{G}$ and $\mathrm{C}$ bases in the reverse Hoogsteen $\mathrm{G}^{*} \cdot \mathrm{C}^{*}(\mathrm{rH})$ base pair around the middle intermolecular (C)N3H $\cdots \mathrm{N} 7(\mathrm{G}) \mathrm{H}$-bond leads to the Hoogsteen $\mathrm{G}^{*} \cdot \mathrm{C}^{*}(\mathrm{H})$ base pair, causing the transformation of the intermolecular specific contacts: (G)O6 $\cdots \mathrm{O} 2(\mathrm{C}),(\mathrm{C}) \mathrm{N} 3 \mathrm{H} \cdots \mathrm{N} 7(\mathrm{G}),(\mathrm{G})$ $\mathrm{C} 8 \mathrm{H} \cdots \mathrm{N} 4(\mathrm{C}) \rightarrow(\mathrm{G}) \mathrm{O} 6 \cdots \mathrm{N} 4(\mathrm{C}),(\mathrm{C}) \mathrm{N} 3 \mathrm{H} \cdots \mathrm{N7}(\mathrm{G}),(\mathrm{G}) \mathrm{C} 8 \mathrm{H} \cdots \mathrm{O} 2(\mathrm{C})$ (Table 1).

3. It was shown that tight $\mathrm{G}^{+} \cdot \mathrm{C}^{-}(\mathrm{rWC})$ ion pair relaxes to the classical Watson-Crick $\mathrm{G} \cdot \mathrm{C}(\mathrm{WC})$ nucleobase pair by the rotation around the middle (G)N1H $\cdots \mathrm{N} 3(\mathrm{C}) \mathrm{H}$-bond, which is assisted by the proton transfer along this $\mathrm{H}$-bond from the $\mathrm{G}^{+}$to $\mathrm{C}^{-}$base. As a result of this transition, it is formed molecular structure, which is not ionic and is characterized by the low value of the imaginary frequency $\left(\nu_{\mathrm{i}}=37.2 \mathrm{~cm}^{-1}\right)$ (Table 1).

4. It was proven that the Watson-Crick $\mathrm{G}^{*} \cdot \mathrm{C}^{*}(\mathrm{WC})$ and reverse Watson-Crick $\mathrm{G}^{*} \cdot \mathrm{C}^{*}(\mathrm{rWC})$ base pairs, from the one side, and the Hoogsteen $\mathrm{G}^{* \mathrm{t}} \cdot \mathrm{C}^{*}(\mathrm{H})$ and reverse Hoogsteen $\mathrm{G}^{* \mathrm{t}} \cdot \mathrm{C}^{*}(\mathrm{rH})$ base pairs, from the other side, turn one into another by the rotations around the middle intermolecular $\mathrm{H}-$ bond: $\mathrm{G}^{*} \cdot \mathrm{C}^{*}(\mathrm{WC}) \leftrightarrow \mathrm{G}^{*} \cdot \mathrm{C}^{*}(\mathrm{rWC})$ and $\mathrm{G}^{* \mathrm{t}} \cdot \mathrm{C}^{*}(\mathrm{H}) \quad \leftrightarrow$ $\mathrm{G}^{* \mathrm{t}} \cdot \mathrm{C}^{*}(\mathrm{rH})$ conformational transitions (Table 1). Conformational transition of the so-called Löwdin $\mathrm{G}^{*} \cdot \mathrm{C}^{*}(\mathrm{WC})$ nucleobase pair, involving $\mathrm{G}^{*}$ and $\mathrm{C}^{*}$ rare tautomers of the nucleobases, occurs through the rotation of the $\mathrm{G}^{*}$ and $\mathrm{C}^{*}$ tautomers around the intermolecular (C)N3H $\cdots \mathrm{N} 1(\mathrm{G}) \mathrm{H}$-bond through the nonplanar $\mathrm{TS}_{\mathrm{G}^{*} \cdot \mathrm{C}^{*}(\mathrm{WC}) \leftrightarrow \mathrm{G}^{*} \cdot \mathrm{C}^{*}(\mathrm{rWC})}$, stabilized by single (C)N3H $\cdots$ N1(G) H-bond (Table 1). This $\mathrm{G}^{*} \cdot \mathrm{C}^{*}(\mathrm{WC}) \leftrightarrow \mathrm{G}^{*} \cdot \mathrm{C}^{*}(\mathrm{rWC})$ conformational transition leads to the so-called reverse Löwdin's $\mathrm{G}^{*} \cdot \mathrm{C} *(\mathrm{rWC})$ nucleobase pair with trans-oriented $\mathrm{N} 1 \mathrm{H}$ and $\mathrm{N} 9 \mathrm{H}$ glycosidic bonds. Geometry of the formed $\mathrm{G}^{*} \cdot \mathrm{C}^{*}(\mathrm{rWC})$ nucleobase pair is Watson-Crick-like. Both Hoogsteen $\mathrm{G}^{* \mathrm{t}} \cdot \mathrm{C}^{*}(\mathrm{H})$ and reverse Hoogsteen $\mathrm{G}^{* \mathrm{t}} \cdot \mathrm{C}^{*}(\mathrm{rH})$ base mispairs conformationally transform into the reverse wobble $\mathrm{G}^{* \mathrm{t}} \cdot \mathrm{C}^{*}\left(\mathrm{rw}_{\mathrm{H}}\right)$ and wobble $\mathrm{G}^{* \mathrm{t}} \cdot \mathrm{C}^{*}\left(\mathrm{w}_{\mathrm{WC} / \mathrm{H}}\right)$ base mispairs, respectively, via the mutual rotations of the bases around the intermolecular H-bonds: $\mathrm{G}^{* \mathrm{t}} \cdot \mathrm{C}^{*}(\mathrm{H}) \leftrightarrow \mathrm{G}^{* \mathrm{t}} \cdot \mathrm{C}^{*}\left(\mathrm{rw}_{\mathrm{H}}\right)$ and $\mathrm{G}^{* \mathrm{t}} \cdot \mathrm{C}^{*}(\mathrm{rH}) \leftrightarrow \mathrm{G}^{* \mathrm{t}} \cdot \mathrm{C}^{*}\left(\mathrm{w}_{\mathrm{WC} / \mathrm{H}}\right)$, respectively.

5. Rotation of the $\mathrm{G}^{*}$ and $\mathrm{C}^{*}$ rare tautomers in the Löwdin's $\mathrm{G}^{*} \cdot \mathrm{C}^{*}(\mathrm{WC})$ nucleobase pair around the upper (G)O6H $\cdots \mathrm{N} 4(\mathrm{C})$ or lower (G) $22 \mathrm{H} \cdots \mathrm{O} 2(\mathrm{C}) \mathrm{H}$-bonds leads to the formation of the two reverse wobble $\mathrm{G}^{*} \cdot \mathrm{C}^{*}\left(\mathrm{rw}_{\mathrm{WC} / \mathrm{H}}\right)$ and $\mathrm{G}^{*} \cdot \mathrm{C}^{*}\left(\mathrm{rw}_{\mathrm{WC}}\right)$ base mispairs, respectively: $\mathrm{G}^{*} \cdot \mathrm{C}^{*}(\mathrm{WC}) \leftrightarrow \mathrm{G}^{*} \cdot \mathrm{C}^{*}\left(\mathrm{rw}_{\mathrm{WC} / \mathrm{H}}\right)$ and $\mathrm{G}^{*} \cdot \mathrm{C}^{*}(\mathrm{WC}) \leftrightarrow \mathrm{G}^{*} \cdot \mathrm{C}^{*}\left(\mathrm{rw}_{\mathrm{WC}}\right)$ conformational transformations, accordingly (Table 1).

6. The same situation is observed for the reverse Löwdin's $\mathrm{G}^{*} \cdot \mathrm{C}^{*}(\mathrm{rWC})$ nucleobase pair: $\mathrm{G}^{*} \cdot \mathrm{C}^{*}(\mathrm{rWC}) \leftrightarrow \mathrm{G}^{*} \cdot \mathrm{C}^{*}\left(\mathrm{~W}_{\mathrm{WC} / \mathrm{H}}\right)$ and $\mathrm{G}^{*} \cdot \mathrm{C}^{*}(\mathrm{rWC}) \leftrightarrow \mathrm{G}^{*} \cdot \mathrm{C}^{*}\left(\mathrm{w}_{\mathrm{WC}}\right)$ conformational transformations, which occur via the mutual rotations of the bases around the upper (G)O6H $\cdots \mathrm{O} 2(\mathrm{C})$ and lower (G) $\mathrm{N} 2 \mathrm{H} \cdots \mathrm{N} 4(\mathrm{C}) \mathrm{H}-$ bonds, respectively, leading to the formation of the wobble 
Table 2 Vibrational, energetic and polar characteristics of the conformational transformations of the $G \cdot C$ nucleobase pairs via the mutual rotations of the bases around the intermolecular $\mathrm{H}$-bonds, obtained at the MP2/6-311++G(2df,pd)//B3LYP/6-311++G(d,p) level of theory in vacuum $(\varepsilon=1)$ under normal conditions $(T=298.15 \mathrm{~K})$ (see Table 1 )

\begin{tabular}{|c|c|c|c|c|c|c|}
\hline Conformational transformation & $\nu_{\mathrm{i} \text { TS }}^{a}$ & $\Delta G^{b}$ & $\Delta E^{c}$ & $\Delta \Delta G_{\mathrm{TS}}{ }^{d}$ & $\Delta \Delta E_{\mathrm{TS}}{ }^{e}$ & $\mu_{\mathrm{TS}}^{f}$ \\
\hline \multicolumn{7}{|c|}{ Watson-Crick (WC), reverse Watson-Crick (rWC), wobble Watson-Crick ( $\left.\mathbf{w}_{\mathrm{WC}}\right)$ and reverse wobble Watson-Crick (rw $\left.\mathrm{wC}_{\mathrm{WC}}\right)$ configurations } \\
\hline $\mathrm{G} \cdot \mathrm{C}(\mathrm{WC}) \leftrightarrow \mathrm{G} \cdot \mathrm{C}\left(\mathrm{rw}_{\mathrm{WC}}\right)$ & 24.2 & 11.53 & 13.09 & 12.63 & 13.30 & 7.94 \\
\hline $\mathrm{G}^{*} \cdot \mathrm{C}^{*}(\mathrm{WC}) \leftrightarrow \mathrm{G}^{*} \cdot \mathrm{C}^{*}(\mathrm{rWC})$ & 37.2 & 2.03 & 2.07 & 13.11 & 14.53 & 4.12 \\
\hline $\mathrm{G}^{*} \cdot \mathrm{C}^{*}(\mathrm{WC}) \leftrightarrow \mathrm{G}^{*} \cdot \mathrm{C}^{*}\left(\mathrm{rw}_{\mathrm{WC} / \mathrm{H}}\right)$ & 17.7 & 7.63 & 10.21 & 10.05 & 11.17 & 6.47 \\
\hline $\mathrm{G}^{*} \cdot \mathrm{C}^{*}(\mathrm{WC}) \leftrightarrow \mathrm{G}^{*} \cdot \mathrm{C}^{*}\left(\mathrm{rw}_{\mathrm{WC}}\right)$ & 30.3 & 11.95 & 14.30 & 12.98 & 14.53 & 5.21 \\
\hline $\mathrm{G} \cdot \mathrm{C}^{*} \mathrm{O} 2(\mathrm{rWC}) \leftrightarrow \mathrm{G} \cdot \mathrm{C}^{*}{ }_{\mathrm{O} 2}\left(\mathrm{~W}_{\mathrm{WC}}\right)$ & 43.4 & 16.74 & 17.33 & 19.04 & 19.92 & 7.45 \\
\hline $\mathrm{G}^{*} \cdot \mathrm{C}^{*}\left(\mathrm{rw}_{\mathrm{WC} / \mathrm{H}}\right) \leftrightarrow \mathrm{G}^{*} \cdot \mathrm{C}^{*}\left(\mathrm{w}_{\mathrm{WC} / \mathrm{H}}\right)$ & 18.8 & 2.80 & 3.02 & 7.49 & 7.16 & 3.94 \\
\hline $\mathrm{G}^{* \mathrm{t}} \cdot \mathrm{C}^{*}\left(\mathrm{rw}_{\mathrm{WC} / \mathrm{H}}\right) \leftrightarrow \mathrm{G}^{* \mathrm{t}} \cdot \mathrm{C}^{*}\left(\mathrm{w}_{\mathrm{WC} / \mathrm{H}}\right)$ & 11.2 & 4.37 & 4.76 & 8.48 & 8.35 & 3.30 \\
\hline $\mathrm{G}^{* \mathrm{t}} \cdot \mathrm{C}^{*}(\mathrm{rWC}) \leftrightarrow \mathrm{G}^{* \mathrm{t}} \cdot \mathrm{C}^{*}(\mathrm{WC})$ & 24.0 & 1.34 & 1.54 & 5.23 & 6.28 & 1.08 \\
\hline $\mathrm{G}^{* \mathrm{t}} \cdot \mathrm{C}^{*}(\mathrm{rWC}) \leftrightarrow \mathrm{G}^{* \mathrm{t}} \cdot \mathrm{C}^{*}\left(\mathrm{w}_{\mathrm{WC}}\right)$ & 14.6 & 2.74 & 4.24 & 4.13 & 3.97 & 2.10 \\
\hline $\mathrm{G} \cdot \mathrm{C}^{* \mathrm{t}}{ }_{\mathrm{O} 2}(\mathrm{rWC}) \leftrightarrow \mathrm{G} \cdot \mathrm{C}^{* \mathrm{t}_{\mathrm{O} 2}}\left(\mathrm{~W}_{\mathrm{WC}}\right)$ & 36.4 & 9.20 & 9.84 & 12.28 & 13.65 & 7.40 \\
\hline $\mathrm{G} \cdot \mathrm{C}^{*}\left(\mathrm{rw}_{\mathrm{WC}}\right)_{\uparrow} \leftrightarrow \mathrm{G} \cdot \mathrm{C}^{*}\left(\mathrm{w}_{\mathrm{WC}}\right)_{\uparrow}$ & 26.5 & 1.38 & 1.55 & 9.98 & 11.47 & 6.10 \\
\hline $\mathrm{G} \cdot \mathrm{C}^{*}\left(\mathrm{rw}_{\mathrm{WC}}\right)_{\uparrow} \leftrightarrow \mathrm{G} \cdot \mathrm{C}^{*}\left(\mathrm{w}_{\mathrm{WC}}\right)$ & 37.4 & 2.75 & 3.05 & 4.34 & 5.80 & 8.68 \\
\hline $\mathrm{G}^{*} \cdot \mathrm{C}\left(\mathrm{rw}_{\mathrm{WC}}\right)_{\uparrow} \leftrightarrow \mathrm{G}^{*} \cdot \mathrm{C}\left(\mathrm{w}_{\mathrm{WC} / \mathrm{H}}\right)$ & 38.1 & 2.29 & 4.06 & 3.73 & 4.71 & 7.39 \\
\hline $\mathrm{G}^{*} \cdot \mathrm{C}\left(\mathrm{rw}_{\mathrm{WC}}\right)_{\uparrow} \leftrightarrow \mathrm{G}^{*} \cdot \mathrm{C}\left(\mathrm{w}_{\mathrm{WC}}\right)_{\downarrow}$ & 29.8 & 3.32 & 4.09 & 8.09 & 9.14 & 6.05 \\
\hline $\mathrm{G}^{*} \cdot \mathrm{C}\left(\mathrm{w}_{\mathrm{WC}}\right)_{\downarrow} \leftrightarrow \mathrm{G}^{*} \cdot \mathrm{C}\left(\mathrm{rw}_{\mathrm{WC}}\right)$ & 36.5 & 2.08 & 2.81 & 2.96 & 2.91 & 5.63 \\
\hline $\mathrm{G}^{*} \cdot \mathrm{C}^{*} \mathrm{O} 2\left(\mathrm{w}_{\mathrm{WC}}\right)_{\uparrow} \leftrightarrow \mathrm{G}^{*} \cdot \mathrm{C}^{*} \mathrm{O} 2\left(\mathrm{rw}_{\mathrm{WC}}\right)_{\downarrow}$ & 42.8 & 3.09 & 3.82 & 12.20 & 11.29 & 4.64 \\
\hline $\mathrm{G}^{*} \cdot \mathrm{C}^{*}{ }_{\mathrm{O} 2}\left(\mathrm{~W}_{\mathrm{WC}}\right)_{\uparrow} \leftrightarrow \mathrm{G}^{*} \cdot \mathrm{C}^{*} \mathrm{O} 2\left(\mathrm{rw}_{\mathrm{WC} / \mathrm{H}}\right)$ & 55.3 & 8.74 & 9.01 & 11.52 & 11.00 & 6.00 \\
\hline $\mathrm{G}^{*} \cdot \mathrm{C}^{*} \mathrm{O} 2\left(\mathrm{rw}_{\mathrm{WC}}\right)_{\downarrow} \leftrightarrow \mathrm{G}^{*} \cdot \mathrm{C}^{*} \mathrm{O} 2\left(\mathrm{w}_{\mathrm{WC}}\right)$ & 87.3 & 7.07 & 6.14 & 9.10 & 7.84 & 4.48 \\
\hline $\mathrm{G}^{*}{ }_{\mathrm{N} 2} \cdot \mathrm{C}^{*}\left(\mathrm{w}_{\mathrm{WC}}\right)_{\downarrow} \leftrightarrow \mathrm{G}^{*}{ }_{\mathrm{N} 2} \cdot \mathrm{C}^{*}\left(\mathrm{rw}_{\mathrm{WC}}\right)_{\downarrow}$ & 36.5 & 1.68 & 1.00 & 13.90 & 15.15 & 2.62 \\
\hline $\mathrm{G}^{* \mathrm{t}} \cdot \mathrm{C}^{*}{ }_{\mathrm{O} 2}\left(\mathrm{rw}_{\mathrm{WC}}\right)_{\downarrow} \leftrightarrow \mathrm{G}^{* \mathrm{t}} \cdot \mathrm{C}^{*}{ }_{\mathrm{O} 2}\left(\mathrm{w}_{\mathrm{WC}}\right)$ & 72.6 & 8.04 & 8.49 & 9.55 & 9.27 & 1.75 \\
\hline $\mathrm{G}^{*} \cdot \mathrm{C}^{* \mathrm{t}}{ }_{\mathrm{O} 2}\left(\mathrm{w}_{\mathrm{WC}}\right)_{\uparrow} \leftrightarrow \mathrm{G}^{*} \cdot \mathrm{C}^{* \mathrm{t}}{ }_{\mathrm{O} 2}\left(\mathrm{rw}_{\mathrm{WC} / \mathrm{H}}\right)$ & 51.0 & 8.10 & 8.45 & 11.26 & 11.09 & 5.39 \\
\hline $\mathrm{G}^{*} \cdot \mathrm{C}^{* \mathrm{t}}{ }_{\mathrm{O} 2}\left(\mathrm{w}_{\mathrm{WC}}\right)_{\uparrow} \leftrightarrow \mathrm{G}^{*} \cdot \mathrm{C}^{* \mathrm{t}}{ }_{\mathrm{O} 2}\left(\mathrm{rw}_{\mathrm{WC}}\right)_{\downarrow}$ & 39.5 & 5.73 & 5.65 & 11.27 & 10.80 & 5.74 \\
\hline
\end{tabular}

\begin{tabular}{|c|c|c|c|c|c|c|}
\hline $\mathrm{G}^{*} \cdot \mathrm{C}^{*}(\mathrm{rH}) \leftrightarrow \mathrm{G}^{*} \cdot \mathrm{C}^{*}(\mathrm{H})$ & 11.3 & 0.76 & 0.75 & 3.58 & 2.90 & 1.45 \\
\hline $\mathrm{G}^{* \mathrm{t}} \cdot \mathrm{C}^{*}(\mathrm{H}) \leftrightarrow \mathrm{G}^{* \mathrm{t}} \cdot \mathrm{C}^{*}(\mathrm{rH})$ & 25.8 & 2.59 & 2.72 & 11.35 & 12.71 & 3.33 \\
\hline $\mathrm{G}^{* \mathrm{t}} \cdot \mathrm{C}^{*}(\mathrm{H}) \leftrightarrow \mathrm{G}^{* \mathrm{t}} \cdot \mathrm{C}^{*}\left(\mathrm{rw}_{\mathrm{H}}\right)$ & 19.5 & 5.28 & 7.08 & 8.37 & 8.98 & 7.54 \\
\hline $\mathrm{G}^{* \mathrm{t}} \cdot \mathrm{C}^{*}(\mathrm{rH}) \leftrightarrow \mathrm{G}^{* \mathrm{t}} \cdot \mathrm{C}^{*}\left(\mathrm{w}_{\mathrm{WC} / \mathrm{H}}\right)$ & 12.0 & 7.05 & 9.12 & 8.79 & 9.31 & 6.73 \\
\hline $\mathrm{G}^{* \mathrm{t}} \cdot \mathrm{C}^{*} \mathrm{O} 2_{2}\left(\mathrm{w}_{\mathrm{H}}\right)_{\uparrow} \leftrightarrow \mathrm{G}^{* \mathrm{t}} \cdot \mathrm{C}^{*} \mathrm{O} 2\left(\mathrm{rw}_{\mathrm{H}}\right)_{\downarrow}$ & 48.0 & 3.93 & 4.69 & 8.53 & 9.22 & 4.25 \\
\hline $\mathrm{G}^{* \mathrm{t}} \cdot \mathrm{C}^{*}{ }_{\mathrm{O} 2}\left(\mathrm{w}_{\mathrm{H}}\right)_{\uparrow} \leftrightarrow \mathrm{G}^{* \mathrm{t}} \cdot \mathrm{C}^{*}{ }_{\mathrm{O} 2}\left(\mathrm{rw}_{\mathrm{H}}\right)$ & 104.7 & 4.84 & 6.47 & 9.87 & 11.67 & 7.58 \\
\hline $\mathrm{G}^{* \mathrm{t}} \cdot \mathrm{C}^{*} \mathrm{O} 2\left(\mathrm{w}_{\mathrm{H}}\right)_{\uparrow} \leftrightarrow \mathrm{G}^{* \mathrm{t}} \cdot \mathrm{C}^{*} \mathrm{O} 2\left(\mathrm{rw}_{\mathrm{WC} / \mathrm{H}}\right)$ & 42.0 & 8.47 & 9.73 & 9.67 & 9.96 & 7.55 \\
\hline $\mathrm{G}^{* \mathrm{t}} \cdot \mathrm{C}^{* \mathrm{t}}{ }_{\mathrm{O} 2}\left(\mathrm{w}_{\mathrm{H}}\right)_{\uparrow} \leftrightarrow \mathrm{G}^{* \mathrm{t}} \cdot \mathrm{C}^{* \mathrm{t}}{ }_{\mathrm{O} 2}\left(\mathrm{rw}_{\mathrm{H}}\right)_{\uparrow}$ & 32.2 & 6.55 & 6.58 & 10.62 & 10.82 & 6.28 \\
\hline $\mathrm{G}^{* \mathrm{t}} \cdot \mathrm{C}^{* \mathrm{t}}{ }_{\mathrm{O} 2}\left(\mathrm{w}_{\mathrm{H}}\right)_{\uparrow} \leftrightarrow \mathrm{G}^{* \mathrm{t}} \cdot \mathrm{C}^{* \mathrm{t}}{ }_{\mathrm{O} 2}\left(\mathrm{rw}_{\mathrm{H}}\right)_{\downarrow}$ & 45.2 & 6.31 & 7.84 & 9.15 & 9.55 & 5.92 \\
\hline $\mathrm{G}^{* \mathrm{t}} \cdot \mathrm{C}^{* \mathrm{t}}{ }_{\mathrm{O} 2}\left(\mathrm{rw}_{\mathrm{H}}\right)_{\downarrow} \leftrightarrow \mathrm{G}^{* \mathrm{t}} \cdot \mathrm{C}^{* \mathrm{t}}{ }_{\mathrm{O} 2}\left(\mathrm{w}_{\mathrm{H}}\right)_{\downarrow}$ & 14.2 & 7.28 & 9.78 & 8.89 & 9.99 & 8.04 \\
\hline $\mathrm{G}^{* \mathrm{t}} \cdot \mathrm{C}\left(\mathrm{w}_{\mathrm{H}}\right)_{\downarrow} \leftrightarrow \mathrm{G}^{* \mathrm{t}} \cdot \mathrm{C}\left(\mathrm{rw}_{\mathrm{H}}\right)$ & 26.7 & 2.45 & 1.23 & 4.74 & 3.84 & 3.41 \\
\hline $\mathrm{G}^{* \mathrm{t}} \cdot \mathrm{C}\left(\mathrm{rw}_{\mathrm{H}}\right)_{\uparrow} \leftrightarrow \mathrm{G}^{* \mathrm{t}} \cdot \mathrm{C}\left(\mathrm{w}_{\mathrm{H}}\right)_{\downarrow}$ & 25.5 & 2.73 & 4.24 & 5.72 & 3.92 & 4.50 \\
\hline $\mathrm{G}^{* \mathrm{t}} \cdot \mathrm{C}\left(\mathrm{rw}_{\mathrm{H}}\right)_{\uparrow} \leftrightarrow \mathrm{G}^{* \mathrm{t}} \cdot \mathrm{C}\left(\mathrm{w}_{\mathrm{H}}\right)_{\uparrow}$ & 32.2 & 2.00 & 3.34 & 3.67 & 3.64 & 8.84 \\
\hline $\mathrm{G}^{*}{ }_{\mathrm{N} 7} \cdot \mathrm{C}^{*}\left(\mathrm{rw}_{\mathrm{H}}\right)_{\uparrow} \leftrightarrow \mathrm{G}^{*}{ }_{\mathrm{N} 7} \cdot \mathrm{C}^{*}\left(\mathrm{w}_{\mathrm{H}}\right)_{\uparrow}$ & 34.7 & 3.25 & 3.12 & 13.36 & 13.06 & 9.49 \\
\hline $\mathrm{G}^{*}{ }_{\mathrm{N} 7} \cdot \mathrm{C}^{*}\left(\mathrm{rw}_{\mathrm{H}}\right)_{\uparrow} \leftrightarrow \mathrm{G}^{*}{ }_{\mathrm{N} 7} \cdot \mathrm{C}^{*}\left(\mathrm{w}_{\mathrm{H}}\right)$ & 19.5 & 4.62 & 4.71 & 8.70 & 7.53 & 4.58 \\
\hline $\mathrm{G}^{*}{ }_{\mathrm{N} 7} \cdot \mathrm{C}^{* \mathrm{t}}\left(\mathrm{w}_{\mathrm{H}}\right)_{\uparrow} \leftrightarrow \mathrm{G}^{*}{ }_{\mathrm{N} 7} \cdot \mathrm{C}^{* \mathrm{t}}\left(\mathrm{rw}_{\mathrm{H}}\right)_{\uparrow}$ & 46.0 & 6.43 & 6.23 & 12.63 & 12.92 & 9.89 \\
\hline $\mathrm{G}^{*} \mathrm{O} / \mathrm{N} 7 \cdot \mathrm{C}^{*}\left(\mathrm{w}_{\mathrm{H}}\right)_{\downarrow} \leftrightarrow \mathrm{G}^{*} \mathrm{O6/ \textrm {N } 7} \cdot \mathrm{C}^{*}\left(\mathrm{rw}_{\mathrm{H}}\right)_{\downarrow}$ & 20.2 & 2.29 & 2.42 & 8.82 & 9.66 & 1.52 \\
\hline $\mathrm{G}^{*}{ }_{\mathrm{O} / \mathrm{N} 7} \cdot \mathrm{C}^{*}\left(\mathrm{w}_{\mathrm{H}}\right)_{\downarrow} \leftrightarrow \mathrm{G}^{*}{ }_{\mathrm{O} / \mathrm{N} 7} \cdot \mathrm{C}^{*}\left(\mathrm{rw}_{\mathrm{H}}\right)_{\uparrow}$ & 15.7 & 4.17 & 4.14 & 8.12 & 8.84 & 5.47 \\
\hline 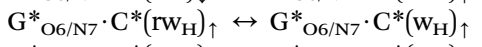 & 16.3 & 1.66 & 1.97 & 6.72 & 7.98 & 4.79 \\
\hline $\mathrm{G}^{*} \mathrm{O} / \mathrm{N} 7 \cdot \mathrm{C}^{*}\left(\mathrm{rw}_{\mathrm{H}}\right)_{\downarrow} \leftrightarrow \mathrm{G}^{*}{ }_{\mathrm{O} 6 / \mathrm{N} 7} \cdot \mathrm{C}^{*}\left(\mathrm{w}_{\mathrm{H}}\right)_{\uparrow}$ & 20.6 & 3.54 & 3.68 & 6.75 & 7.56 & 3.19 \\
\hline $\mathrm{G}^{* \mathrm{t}} \mathrm{\textrm {O } / \mathrm { N } \mathrm { N }} \cdot \mathrm{C}^{*}\left(\mathrm{w}_{\mathrm{H}}\right)_{\downarrow} \leftrightarrow \mathrm{G}^{* \mathrm{O}^{\mathrm{t}} / \mathrm{N} 7} \cdot \mathrm{C}^{*}\left(\mathrm{rw}_{\mathrm{H}}\right)_{\downarrow}$ & 18.1 & 2.25 & 2.28 & 10.40 & 11.67 & 2.71 \\
\hline
\end{tabular}

${ }^{a}$ Imaginary frequency at the TS of the conformational transformation, $\mathrm{cm}^{-1} \cdot{ }^{b}$ Relative Gibbs free energy of the formed $\mathrm{G} \cdot \mathrm{C}$ nucleobase pair $(T=$ $298.15 \mathrm{~K}$ ), $\mathrm{kcal} \mathrm{mol}{ }^{-1} \cdot{ }^{c}$ Relative electronic energy of the formed $\mathrm{G} \cdot \mathrm{C}$ nucleobase pair, $\mathrm{kcal} \mathrm{mol}{ }^{-1} \cdot{ }^{d}$ Relative Gibbs free energy of the TS of the conformational transformation $(T=298.15 \mathrm{~K}), \mathrm{kcal} \mathrm{mol}^{-1}$. ${ }^{e}$ Relative electronic energy of the TS of the conformational transformation, $\mathrm{kcal} \mathrm{mol}^{-1} \cdot{ }^{f}$ Dipole moment of the TS, D. 
$\mathrm{G}^{*} \cdot \mathrm{C}^{*}\left(\mathrm{w}_{\mathrm{WC} / \mathrm{H}}\right)$ and $\mathrm{G}^{*} \cdot \mathrm{C}^{*}\left(\mathrm{w}_{\mathrm{WC}}\right)$ base mispairs, accordingly (Table 1).

7. The $\mathrm{G} \cdot \mathrm{C}^{*}{ }_{\mathrm{O} 2}(\mathrm{rWC}) \leftrightarrow \mathrm{G} \cdot \mathrm{C}^{*}{ }_{\mathrm{O} 2}\left(\mathrm{~W}_{\mathrm{WC}}\right)$ conformational transformation of the reverse Watson-Crick $\mathrm{G} \cdot \mathrm{C}^{*}{ }_{\mathrm{O} 2}(\mathrm{rWC})$ nucleobase pair by the participation of the $\mathrm{G}$ base and $\mathrm{C}_{\mathrm{O} 2}$ tautomer of the $\mathrm{C}$ nucleobase proceeds through the rotation of the $\mathrm{G}$ and $\mathrm{C}^{*} \mathrm{O} 2$ bases around the middle $(\mathrm{G}) \mathrm{N} 1 \mathrm{H} \cdots \mathrm{N} 3(\mathrm{C}) \mathrm{H}$-bond and leads to the formation of the wobble $\mathrm{G} \cdot \mathrm{C}^{*}{ }_{\mathrm{O} 2}\left(\mathrm{w}_{\mathrm{WC}}\right)$ base pair, which has non-planar geometry. So, this conformational transformation greatly changes the geometry of the initial $\mathrm{G} \cdot \mathrm{C}^{*}{ }_{\mathrm{O} 2}(\mathrm{rWC})$ base pair from reverse Watson-Crick geometry to wobble Watson-Crick geometry.

8. The $\mathrm{G}^{*} \cdot \mathrm{C}^{*}\left(\mathrm{rw}_{\mathrm{WC} / \mathrm{H}}\right)$ and $\mathrm{G}^{*} \cdot \mathrm{C}^{*}\left(\mathrm{w}_{\mathrm{WC} / \mathrm{H}}\right)$ base mispairs mutually transform into each other through the mutual rotation of the bases around the intermolecular lower (C)N3H $\cdots \mathrm{O} 6(\mathrm{G}) \mathrm{H}$ bond: $\mathrm{G}^{*} \cdot \mathrm{C}^{*}\left(\mathrm{rw}_{\mathrm{WC} / \mathrm{H}}\right) \leftrightarrow \mathrm{G}^{*} \cdot \mathrm{C}^{*}\left(\mathrm{w}_{\mathrm{WC} / \mathrm{H}}\right)$ (Table 1). Similar transformation occurs also for the $\mathrm{G}^{* \mathrm{t}} \cdot \mathrm{C}^{*}\left(\mathrm{rw}_{\mathrm{WC} / \mathrm{H}}\right)$ and $\mathrm{G}^{* \mathrm{t}} \cdot \mathrm{C}^{*}\left(\mathrm{w}_{\mathrm{WC} / \mathrm{H}}\right)$ base mispairs by the participation of the $\mathrm{G}^{* \mathrm{t}}$ base with trans-oriented $\mathrm{O} 6 \mathrm{H}$ hydroxyl group: $\mathrm{G}^{* \mathrm{t}} \cdot \mathrm{C}^{*}\left(\mathrm{rw}_{\mathrm{WC} / \mathrm{H}}\right)$ $\leftrightarrow \mathrm{G}^{* \mathrm{t}} \cdot \mathrm{C}^{*}\left(\mathrm{w}_{\mathrm{WC} / \mathrm{H}}\right)$ (Table 1$)$.

9. The $\mathrm{G}^{* \mathrm{t}} \cdot \mathrm{C}^{*}(\mathrm{rWC}) \leftrightarrow \mathrm{G}^{* \mathrm{t}} \cdot \mathrm{C}^{*}(\mathrm{WC})$ and $\mathrm{G}^{* \mathrm{t}} \cdot \mathrm{C}^{*}(\mathrm{rWC}) \leftrightarrow$ $\mathrm{G}^{* \mathrm{t}} \cdot \mathrm{C}^{*}\left(\mathrm{w}_{\mathrm{WC}}\right)$ conformational transformations of the reverse Watson-Crick $\mathrm{G}^{* \mathrm{t}} \cdot \mathrm{C}^{*}(\mathrm{rWC})$ base pair occur via the mutual rotations of the bases around the middle $(\mathrm{C}) \mathrm{N} 3 \mathrm{H} \cdots \mathrm{N} 1(\mathrm{G})$ and lower (G)N2H $\cdots \mathrm{N} 4(\mathrm{C}) \mathrm{H}$-bonds, respectively, and lead to the

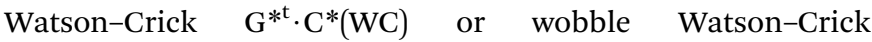
$\mathrm{G}^{* \mathrm{t}} \cdot \mathrm{C}^{*}\left(\mathrm{~W}_{\mathrm{WC}}\right)$ nucleobase mispairs, respectively (Table 1$)$. The $\mathrm{G} \cdot \mathrm{C}^{* \mathrm{t}}{ }_{\mathrm{O} 2}(\mathrm{rWC}) \leftrightarrow \mathrm{G} \cdot \mathrm{C}^{* \mathrm{t}}{ }_{\mathrm{O} 2}\left(\mathrm{w}_{\mathrm{WC}}\right)$ conformational transformation proceeds for the reverse Watson-Crick $\mathrm{G} \cdot \mathrm{C}^{* \mathrm{t}}{ }_{\mathrm{O} 2}(\mathrm{rWC})$ base mispair by the participation of the $\mathrm{G}$ base and $\mathrm{C}^{* \mathrm{t}}{ }_{\mathrm{O} 2}$ tautomer with trans-oriented $\mathrm{N} 4 \mathrm{H}$ imino group and leads to the wobble Watson-Crick $\mathrm{G} \cdot \mathrm{C}^{* \mathrm{t}}{ }_{\mathrm{O} 2}\left(\mathrm{w}_{\mathrm{WC}}\right)$ base mispair (Table 1$)$.

10. It is especially interesting to consider the conformational transformations of the novel, unusual Watson-Crick $\mathrm{G}^{* \mathrm{t}} \cdot \mathrm{C}^{* \mathrm{t}}(\mathrm{WC})$ and reverse Watson-Crick $\mathrm{G}^{* \mathrm{t}} \cdot \mathrm{C}^{*}(\mathrm{rWC})$ base pairs by the participation of the $\mathrm{G}^{* \mathrm{t}}$ and $\mathrm{C}^{* \mathrm{t}}$ rare tautomers with transoriented hydroxyl group of the $\mathrm{G}^{* \mathrm{t}}$ base and trans-oriented imino group of the $\mathrm{C}^{* \mathrm{t}}$ base, respectively.

Thus, the $\mathrm{G}^{* \mathrm{t}} \cdot \mathrm{C}^{* \mathrm{t}}(\mathrm{WC}) \leftrightarrow \mathrm{G}^{* \mathrm{t}} \cdot \mathrm{C}^{* \mathrm{t}}\left(\mathrm{rw}_{\mathrm{WC}}\right)_{\uparrow}$ and $\mathrm{G}^{* \mathrm{t}} \cdot \mathrm{C}^{* \mathrm{t}}(\mathrm{WC})$ $\leftrightarrow \mathrm{G}^{* \mathrm{t}} \cdot \mathrm{C}^{* \mathrm{t}}\left(\mathrm{rw}_{\mathrm{WC}}\right)$ conformational transformations of the $\mathrm{G}^{* \mathrm{t}} \cdot \mathrm{C}^{* \mathrm{t}}(\mathrm{WC})$ base mispair via the mutual rotations of the bases around the middle $(\mathrm{C}) \mathrm{N} 3 \mathrm{H} \cdots \mathrm{N} 1(\mathrm{G})$ or lower $(\mathrm{G}) \mathrm{N} 2 \mathrm{H} \cdots \mathrm{O} 2(\mathrm{C}) \mathrm{H}-$ bonds lead to the formation of the reverse wobble $\mathrm{G}^{* \mathrm{t}} \cdot \mathrm{C}^{* \mathrm{t}}\left(\mathrm{rw}_{\mathrm{WC}}\right)_{\uparrow}$ or $\mathrm{G}^{* \mathrm{t}} \cdot \mathrm{C}^{* \mathrm{t}}\left(\mathrm{rw}_{\mathrm{WC}}\right)$ base pairs, respectively (Table 1). It was also revealed mutual conformational transformations between formed wobble base mispairs.

11. In general, conformational transformations of the $\mathrm{G} \cdot \mathrm{C}$ base pairs in Watson-Crick (WC), reverse Watson-Crick (rWC), wobble Watson-Crick ( $\mathrm{w}_{\mathrm{WC}}$ ) and reverse wobble Watson-Crick $\left(\mathrm{rw}_{\mathrm{WC}}\right)$ configurations occur via the mutual rotation of the bases:

- around the upper H-bond: $\mathrm{G}^{*} \cdot \mathrm{C}^{*}(\mathrm{WC}) \leftrightarrow \mathrm{G}^{*} \cdot \mathrm{C}^{*}\left(\mathrm{rw}_{\mathrm{WC} / \mathrm{H}}\right)$, $\mathrm{G}^{*} \cdot \mathrm{C}^{*}(\mathrm{rWC}) \leftrightarrow \mathrm{G}^{*} \cdot \mathrm{C}^{*}\left(\mathrm{w}_{\mathrm{WC} / \mathrm{H}}\right), \mathrm{G}^{* \mathrm{t}} \cdot \mathrm{C}^{*}\left(\mathrm{rw}_{\mathrm{WC} / \mathrm{H}}\right) \leftrightarrow \mathrm{G}^{* \mathrm{t}} \cdot \mathrm{C}^{*}\left(\mathrm{w}_{\mathrm{WC} /}\right.$ $\mathrm{H}), \mathrm{G} \cdot \mathrm{C}^{*}\left(\mathrm{rw}_{\mathrm{WC}}\right)_{\uparrow} \leftrightarrow \mathrm{G} \cdot \mathrm{C}^{*}\left(\mathrm{w}_{\mathrm{WC}}\right)_{\uparrow}, \mathrm{G}^{*} \cdot \mathrm{C}\left(\mathrm{rw}_{\mathrm{WC}}\right)_{\uparrow} \leftrightarrow \mathrm{G}^{*} \cdot \mathrm{C}\left(\mathrm{w}_{\mathrm{WC} / \mathrm{H}}\right)$, $\mathrm{G}^{*} \cdot \mathrm{C}^{*}{ }_{\mathrm{O} 2}\left(\mathrm{w}_{\mathrm{WC}}\right)_{\uparrow} \leftrightarrow \mathrm{G}^{*} \cdot \mathrm{C}^{*}{ }_{\mathrm{O} 2}\left(\mathrm{rw}_{\mathrm{WC} / \mathrm{H}}\right), \mathrm{G}^{*} \cdot \mathrm{C}^{* \mathrm{t}}{ }_{\mathrm{O} 2}\left(\mathrm{w}_{\mathrm{WC}}\right)_{\uparrow} \leftrightarrow$ $\mathrm{G}^{*} \cdot \mathrm{C}^{* \mathrm{t}} \mathrm{O} 2\left(\mathrm{rw}_{\mathrm{WC} / \mathrm{H}}\right), \quad \mathrm{G}^{* \mathrm{t}}{ }_{\mathrm{N} 2} \cdot \mathrm{C}^{*}\left(\mathrm{rw}_{\mathrm{WC}}\right)_{\downarrow} \leftrightarrow \mathrm{G}^{* \mathrm{t}}{ }_{\mathrm{N} 2} \cdot \mathrm{C}^{*}\left(\mathrm{w}_{\mathrm{WC}}\right)_{\uparrow}$, $\mathrm{G}^{* \mathrm{t}}{ }_{\mathrm{N} 2} \cdot \mathrm{C}^{* \mathrm{t}}\left(\mathrm{rw}_{\mathrm{WC}}\right)_{\downarrow} \leftrightarrow \mathrm{G}^{* \mathrm{t}}{ }_{\mathrm{N} 2} \cdot \mathrm{C}^{* \mathrm{t}}\left(\mathrm{w}_{\mathrm{WC}}\right)_{\uparrow}$;
- around the middle H-bond: $\mathrm{G} \cdot \mathrm{C}(\mathrm{WC}) \leftrightarrow \mathrm{G} \cdot \mathrm{C}\left(\mathrm{rw}_{\mathrm{WC}}\right)$, $\mathrm{G}^{+} \cdot \mathrm{C}^{-}(\mathrm{rWC}) \leftrightarrow \mathrm{G} \cdot \mathrm{C}(\mathrm{WC}), \mathrm{G}^{*} \cdot \mathrm{C}^{*}(\mathrm{WC}) \leftrightarrow \mathrm{G}^{*} \cdot \mathrm{C}^{*}(\mathrm{rWC})$, $\mathrm{G} \cdot \mathrm{C}^{*}{ }_{\mathrm{O} 2}(\mathrm{rWC}) \leftrightarrow \mathrm{G} \cdot \mathrm{C}^{*}{ }_{\mathrm{O} 2}\left(\mathrm{~W}_{\mathrm{WC}}\right), \mathrm{G}^{* \mathrm{t}} \cdot \mathrm{C}^{*}(\mathrm{rWC}) \leftrightarrow \mathrm{G}^{* \mathrm{t}} \cdot \mathrm{C}^{*}(\mathrm{WC})$, $\mathrm{G} \cdot \mathrm{C}^{* \mathrm{t}}{ }_{\mathrm{O} 2}(\mathrm{rWC}) \leftrightarrow \mathrm{G} \cdot \mathrm{C}^{* \mathrm{t}}{ }_{\mathrm{O} 2}\left(\mathrm{~W}_{\mathrm{WC}}\right), \quad \mathrm{G}^{* \mathrm{t}} \cdot \mathrm{C}^{* \mathrm{t}}(\mathrm{WC}) \leftrightarrow$ $\mathrm{G}^{* \mathrm{t}} \cdot \mathrm{C}^{* \mathrm{t}}\left(\mathrm{rw}_{\mathrm{WC}}\right)_{\uparrow}$;

- around the lower H-bond: $\mathrm{G}^{*} \cdot \mathrm{C}^{*}(\mathrm{WC}) \leftrightarrow \mathrm{G}^{*} \cdot \mathrm{C}^{*}\left(\mathrm{rw}_{\mathrm{WC}}\right)$, $\mathrm{G}^{*} \cdot \mathrm{C}^{*}(\mathrm{rWC}) \leftrightarrow \mathrm{G}^{*} \cdot \mathrm{C}^{*}\left(\mathrm{w}_{\mathrm{WC}}\right), \mathrm{G}^{*} \cdot \mathrm{C}^{*}\left(\mathrm{rw}_{\mathrm{WC} / \mathrm{H}}\right) \leftrightarrow \mathrm{G}^{*} \cdot \mathrm{C}^{*}\left(\mathrm{w}_{\mathrm{WC} / \mathrm{H}}\right)$, $\mathrm{G}^{* \mathrm{t}} \cdot \mathrm{C}^{*}(\mathrm{rWC}) \leftrightarrow \mathrm{G}^{* \mathrm{t}} \cdot \mathrm{C}^{*}\left(\mathrm{w}_{\mathrm{WC}}\right), \mathrm{G}^{* \mathrm{t}} \cdot \mathrm{C}^{* \mathrm{t}}(\mathrm{WC}) \leftrightarrow \mathrm{G}^{* \mathrm{t}} \cdot \mathrm{C}^{* \mathrm{t}}\left(\mathrm{rw}_{\mathrm{WC}}\right)$, $\mathrm{G} \cdot \mathrm{C}^{*}\left(\mathrm{w}_{\mathrm{WC}}\right)_{\uparrow} \leftrightarrow \mathrm{G} \cdot \mathrm{C}^{*}\left(\mathrm{rw}_{\mathrm{WC}}\right), \mathrm{G} \cdot \mathrm{C}^{*}\left(\mathrm{rw}_{\mathrm{WC}}\right) \leftrightarrow \mathrm{G} \cdot \mathrm{C}^{*}\left(\mathrm{w}_{\mathrm{WC}}\right)$, $\mathrm{G} \cdot \mathrm{C}^{*}\left(\mathrm{rw}_{\mathrm{WC}}\right)_{\uparrow} \leftrightarrow \mathrm{G} \cdot \mathrm{C}^{*}\left(\mathrm{w}_{\mathrm{WC}}\right), \mathrm{G}^{*} \cdot \mathrm{C}\left(\mathrm{rw}_{\mathrm{WC}}\right)_{\uparrow} \leftrightarrow \mathrm{G}^{*} \cdot \mathrm{C}\left(\mathrm{w}_{\mathrm{WC}}\right)_{\downarrow}$, $\mathrm{G}^{*} \cdot \mathrm{C}\left(\mathrm{w}_{\mathrm{WC}}\right)_{\downarrow} \leftrightarrow \mathrm{G}^{*} \cdot \mathrm{C}\left(\mathrm{rw}_{\mathrm{WC}}\right), \mathrm{G}^{*} \cdot \mathrm{C}^{*} \mathrm{O} 2_{2}\left(\mathrm{w}_{\mathrm{WC}}\right)_{\uparrow} \leftrightarrow \mathrm{G}^{*} \cdot \mathrm{C}^{*} \mathrm{O} 2$ $\left(\mathrm{rw}_{\mathrm{WC}}\right)_{\downarrow}, \mathrm{G}^{*} \cdot \mathrm{C}^{*}{ }_{\mathrm{O} 2}\left(\mathrm{rw}_{\mathrm{WC}}\right)_{\downarrow} \leftrightarrow \mathrm{G}^{*} \cdot \mathrm{C}^{*}{ }_{\mathrm{O} 2}\left(\mathrm{w}_{\mathrm{WC}}\right), \mathrm{G}^{*}{ }_{\mathrm{N} 2} \cdot \mathrm{C}^{*}\left(\mathrm{w}_{\mathrm{WC}}\right)_{\downarrow} \leftrightarrow$ $\mathrm{G}^{*}{ }_{\mathrm{N} 2} \cdot \mathrm{C}^{*}\left(\mathrm{rw}_{\mathrm{WC}}\right)_{\downarrow}, \quad \mathrm{G}^{* \mathrm{t}} \cdot \mathrm{C}^{*}{ }_{\mathrm{O} 2}\left(\mathrm{rw}_{\mathrm{WC}}\right)_{\downarrow} \leftrightarrow \mathrm{G}^{* \mathrm{t}} \cdot \mathrm{C}^{*}{ }_{\mathrm{O} 2}\left(\mathrm{w}_{\mathrm{WC}}\right)$, $\mathrm{G}^{*} \cdot \mathrm{C}^{* \mathrm{t}}{ }_{\mathrm{O} 2}\left(\mathrm{w}_{\mathrm{WC}}\right)_{\uparrow} \leftrightarrow \mathrm{G}^{*} \cdot \mathrm{C}^{* \mathrm{t}}{ }_{\mathrm{O} 2}\left(\mathrm{rw}_{\mathrm{WC}}\right)_{\downarrow}, \mathrm{G}^{*} \cdot \mathrm{C}^{* \mathrm{t}}{ }_{\mathrm{O} 2}\left(\mathrm{rw}_{\mathrm{WC}}\right)_{\downarrow} \leftrightarrow$ $\mathrm{G}^{*} \cdot \mathrm{C}^{* \mathrm{t}}{ }_{\mathrm{O} 2}\left(\mathrm{w}_{\mathrm{WC}}\right), \quad \mathrm{G}^{* \mathrm{t}} \cdot \mathrm{C}^{* \mathrm{t}}{ }_{\mathrm{O} 2}\left(\mathrm{rw}_{\mathrm{WC}}\right)_{\downarrow} \leftrightarrow \mathrm{G}^{* \mathrm{t}} \cdot \mathrm{C}^{* \mathrm{t}}{ }_{\mathrm{O} 2}\left(\mathrm{w}_{\mathrm{WC}}\right)$, $\mathrm{G}^{* \mathrm{t}}{ }_{\mathrm{N} 2} \cdot \mathrm{C}^{*}\left(\mathrm{w}_{\mathrm{WC}}\right)_{\downarrow} \leftrightarrow \mathrm{G}^{* \mathrm{t}}{ }_{\mathrm{N} 2} \cdot \mathrm{C}^{*}\left(\mathrm{rw}_{\mathrm{WC}}\right)_{\downarrow}$.

$\mathrm{G} \cdot \mathrm{C}$ nucleobase pairs in the WC, rWC, $\mathrm{w}_{\mathrm{WC}}$ and $\mathrm{rw}_{\mathrm{WC}}$ configurations are connected by the $\mathrm{NH} \cdots \mathrm{O}, \mathrm{NH} \cdots \mathrm{N}, \mathrm{OH} \cdots \mathrm{N}$, $\mathrm{OH} \cdots \mathrm{O} \mathrm{H}$-bonds and attractive $\mathrm{O} \cdots \mathrm{O} / \mathrm{O} \cdots \mathrm{N}$ van der Waals contacts.

Among the WC, rWC, $\mathrm{w}_{\mathrm{WC}}$ and $\mathrm{rw}_{\mathrm{WC}} \mathrm{G} \cdot \mathrm{C}$ base pairs the following complexes have significantly non-planar geometry: $\mathrm{G} \cdot \mathrm{C}\left(\mathrm{rw}_{\mathrm{WC}}\right), \quad \mathrm{G}^{*} \cdot \mathrm{C}^{*}\left(\mathrm{rw}_{\mathrm{WC}}\right), \quad \mathrm{G}^{*} \cdot \mathrm{C}^{*}\left(\mathrm{w}_{\mathrm{WC}}\right), \quad \mathrm{G}^{* \mathrm{t}} \cdot \mathrm{C}^{* \mathrm{t}}\left(\mathrm{rw}_{\mathrm{WC}}\right)$, $\mathrm{G}^{* \mathrm{t}} \cdot \mathrm{C}^{*}(\mathrm{rWC}), \quad \mathrm{G}^{* \mathrm{t}} \cdot \mathrm{C}^{*}(\mathrm{WC}), \quad \mathrm{G}^{* \mathrm{t}} \cdot \mathrm{C}^{*}\left(\mathrm{w}_{\mathrm{WC}}\right), \quad \mathrm{G} \cdot \mathrm{C}^{*}{ }_{\mathrm{O} 2}\left(\mathrm{~W}_{\mathrm{WC}}\right)$, $\mathrm{G} \cdot \mathrm{C}^{* \mathrm{t}}{ }_{\mathrm{O} 2}(\mathrm{rWC}), \quad \mathrm{G} \cdot \mathrm{C}^{* \mathrm{t}}{ }_{\mathrm{O} 2}\left(\mathrm{w}_{\mathrm{WC}}\right), \quad \mathrm{G} \cdot \mathrm{C}^{*}\left(\mathrm{rw}_{\mathrm{WC}}\right), \quad \mathrm{G} \cdot \mathrm{C}^{*}\left(\mathrm{w}_{\mathrm{WC}}\right)$, $\mathrm{G}^{*} \cdot \mathrm{C}\left(\mathrm{rw}_{\mathrm{WC}}\right)_{\uparrow}, \quad \mathrm{G}^{*} \cdot \mathrm{C}\left(\mathrm{w}_{\mathrm{WC} / \mathrm{H}}\right), \quad \mathrm{G}^{*} \cdot \mathrm{C}\left(\mathrm{w}_{\mathrm{WC}}\right)_{\downarrow}, \quad \mathrm{G}^{*} \cdot \mathrm{C}\left(\mathrm{rw}_{\mathrm{WC}}\right)$, $\mathrm{G}^{*} \cdot \mathrm{C}^{*}{ }_{\mathrm{O} 2}\left(\mathrm{w}_{\mathrm{WC}}\right), \mathrm{G}^{* \mathrm{t}} \cdot \mathrm{C}^{*}{ }_{\mathrm{O} 2}\left(\mathrm{w}_{\mathrm{WC}}\right), \mathrm{G}^{*} \cdot \mathrm{C}^{* \mathrm{t}} \mathrm{O} 2_{2}\left(\mathrm{w}_{\mathrm{WC}}\right)$ and $\mathrm{G}^{* \mathrm{t}} \cdot \mathrm{C}^{* \mathrm{t}}{ }_{\mathrm{O} 2}$ $\left(\mathrm{w}_{\mathrm{WC}}\right)$. This non-planarity of the base pairs is caused by the $\mathrm{sp}^{3}$ hybridization of the $\mathrm{NH}_{2}$ amino group of the $\mathrm{G}$ base and its nonplanarity.

12. Conformational transitions of the $\mathrm{G} \cdot \mathrm{C}$ nucleobase pairs in $\mathrm{H}, \mathrm{rH}, \mathrm{w}_{\mathrm{H}}$ and $\mathrm{rw}_{\mathrm{H}}$ configurations proceed through the mutual rotations of the bases:

- around the upper H-bond: $\mathrm{G}^{* \mathrm{t}} \cdot \mathrm{C}^{*}(\mathrm{H}) \leftrightarrow \mathrm{G}^{* \mathrm{t}} \cdot \mathrm{C}^{*}\left(\mathrm{rw}_{\mathrm{H}}\right)$, $\mathrm{G}^{* \mathrm{t}} \cdot \mathrm{C}^{*}(\mathrm{rH}) \leftrightarrow \mathrm{G}^{* \mathrm{t}} \cdot \mathrm{C}^{*}\left(\mathrm{w}_{\mathrm{WC} / \mathrm{H}}\right), \mathrm{G}^{* \mathrm{t}} \cdot \mathrm{C}^{*} \mathrm{O} 2\left(\mathrm{w}_{\mathrm{H}}\right)_{\uparrow} \leftrightarrow \mathrm{G}^{* \mathrm{t}} \cdot \mathrm{C}^{*} \mathrm{O} 2$ $\left(\mathrm{rw}_{\mathrm{H}}\right), \mathrm{G}^{* \mathrm{t}} \cdot \mathrm{C}^{*}{ }_{\mathrm{O} 2}\left(\mathrm{w}_{\mathrm{H}}\right)_{\uparrow} \leftrightarrow \mathrm{G}^{* \mathrm{t}} \cdot \mathrm{C}^{*}{ }_{\mathrm{O} 2}\left(\mathrm{rw}_{\mathrm{WC} / \mathrm{H}}\right), \mathrm{G}^{* \mathrm{t}} \cdot \mathrm{C}\left(\mathrm{w}_{\mathrm{H}}\right)_{\downarrow} \leftrightarrow$ $\mathrm{G}^{* \mathrm{t}} \cdot \mathrm{C}\left(\mathrm{rw}_{\mathrm{H}}\right), \mathrm{G}^{* \mathrm{t}} \cdot \mathrm{C}\left(\mathrm{rw}_{\mathrm{H}}\right)_{\uparrow} \leftrightarrow \mathrm{G}^{* \mathrm{t}} \cdot \mathrm{C}\left(\mathrm{w}_{\mathrm{H}}\right)_{\uparrow}, \mathrm{G}^{*}{ }_{\mathrm{N} 7} \cdot \mathrm{C}^{*}\left(\mathrm{rw}_{\mathrm{H}}\right)_{\uparrow} \leftrightarrow$ $\mathrm{G}^{*}{ }_{\mathrm{N} 7} \cdot \mathrm{C}^{*}\left(\mathrm{w}_{\mathrm{H}}\right)_{\uparrow}, \quad \mathrm{G}^{*}{ }_{\mathrm{O} 6 / \mathrm{N} 7} \cdot \mathrm{C}^{*}\left(\mathrm{w}_{\mathrm{H}}\right)_{\downarrow} \leftrightarrow \mathrm{G}^{*}{ }_{\mathrm{O} 6 / \mathrm{N} 7} \cdot \mathrm{C}^{*}\left(\mathrm{rw}_{\mathrm{H}}\right)_{\uparrow}$, $\mathrm{G}^{*}{ }_{\mathrm{O} 6 / \mathrm{N} 7} \cdot \mathrm{C}^{*}\left(\mathrm{rw}_{\mathrm{H}}\right)_{\uparrow} \leftrightarrow \mathrm{G}^{*}{ }_{\mathrm{O} 6 / \mathrm{N} 7} \cdot \mathrm{C}^{*}\left(\mathrm{w}_{\mathrm{H}}\right)_{\uparrow}, \mathrm{G}^{*}{ }_{\mathrm{O} 6 / \mathrm{N} 7} \cdot \mathrm{C}^{*}\left(\mathrm{rw}_{\mathrm{H}}\right)_{\downarrow} \leftrightarrow$ $\mathrm{G}^{*}{ }_{\mathrm{O} 6 / \mathrm{N} 7} \cdot \mathrm{C}^{*}\left(\mathrm{w}_{\mathrm{H}}\right)_{\uparrow}$;

- around the middle H-bond: $\mathrm{G}^{*} \cdot \mathrm{C}^{*}(\mathrm{rH}) \leftrightarrow \mathrm{G}^{*} \cdot \mathrm{C}^{*}(\mathrm{H})$, $\mathrm{G}^{* \mathrm{t}} \cdot \mathrm{C}^{*}(\mathrm{H}) \leftrightarrow \mathrm{G}^{* \mathrm{t}} \cdot \mathrm{C}^{*}(\mathrm{rH}), \mathrm{G}^{* \mathrm{t}} \cdot \mathrm{C}^{* \mathrm{t}}{ }_{\mathrm{O} 2}\left(\mathrm{w}_{\mathrm{H}}\right)_{\uparrow} \leftrightarrow \mathrm{G}^{* \mathrm{t}} \cdot \mathrm{C}^{* \mathrm{t}}{ }_{\mathrm{O} 2}\left(\mathrm{rw}_{\mathrm{H}}\right)_{\uparrow}$, $\mathrm{G}^{*}{ }_{\mathrm{N} 7} \cdot \mathrm{C}^{* \mathrm{t}}\left(\mathrm{w}_{\mathrm{H}}\right)_{\uparrow} \leftrightarrow \mathrm{G}^{*}{ }_{\mathrm{N} 7} \cdot \mathrm{C}^{* \mathrm{t}}\left(\mathrm{rw}_{\mathrm{H}}\right)_{\uparrow}$;

- around the lower H-bond: $\mathrm{G}^{* \mathrm{t}} \cdot \mathrm{C}^{*} \mathrm{O} 2_{2}\left(\mathrm{w}_{\mathrm{H}}\right)_{\uparrow} \leftrightarrow \mathrm{G}^{* \mathrm{t}} \cdot \mathrm{C}^{*} \mathrm{O} 2$ $\left(\mathrm{rw}_{\mathrm{H}}\right)_{\downarrow}, \mathrm{G}^{* \mathrm{t}} \cdot \mathrm{C}^{* \mathrm{t}}{ }_{\mathrm{O} 2}\left(\mathrm{w}_{\mathrm{H}}\right)_{\uparrow} \leftrightarrow \mathrm{G}^{* \mathrm{t}} \cdot \mathrm{C}^{* \mathrm{t}}{ }_{\mathrm{O} 2}\left(\mathrm{rw}_{\mathrm{H}}\right)_{\downarrow}, \mathrm{G}^{* \mathrm{t}} \cdot \mathrm{C}^{* \mathrm{t}}{ }_{\mathrm{O} 2}\left(\mathrm{rw}_{\mathrm{H}}\right)_{\downarrow} \leftrightarrow$ $\mathrm{G}^{* \mathrm{t}} \cdot \mathrm{C}^{* \mathrm{t}}{ }_{\mathrm{O} 2}\left(\mathrm{w}_{\mathrm{H}}\right)_{\downarrow}, \mathrm{G}^{* \mathrm{t}} \cdot \mathrm{C}\left(\mathrm{rw}_{\mathrm{H}}\right)_{\uparrow} \leftrightarrow \mathrm{G}^{* \mathrm{t}} \cdot \mathrm{C}\left(\mathrm{w}_{\mathrm{H}}\right)_{\downarrow}, \mathrm{G}^{*}{ }_{\mathrm{N} 7} \cdot \mathrm{C}^{*}\left(\mathrm{rw}_{\mathrm{H}}\right)_{\uparrow} \leftrightarrow$ $\mathrm{G}^{*}{ }_{\mathrm{N} 7} \cdot \mathrm{C}^{*}\left(\mathrm{w}_{\mathrm{H}}\right), \quad \mathrm{G}^{*}{ }_{\mathrm{O} 6 / \mathrm{N} 7} \cdot \mathrm{C}^{*}\left(\mathrm{w}_{\mathrm{H}}\right)_{\downarrow} \leftrightarrow \mathrm{G}^{*}{ }_{\mathrm{O} 6 / \mathrm{N} 7} \cdot \mathrm{C}^{*}\left(\mathrm{rw}_{\mathrm{H}}\right)_{\downarrow}$, $\mathrm{G}^{* \mathrm{t}}{ }_{\mathrm{O} 6 / \mathrm{N} 7} \cdot \mathrm{C}^{*}\left(\mathrm{w}_{\mathrm{H}}\right)_{\downarrow} \leftrightarrow \mathrm{G}^{* \mathrm{t}}{ }_{\mathrm{O} 6 / \mathrm{N} 7} \cdot \mathrm{C}^{*}\left(\mathrm{rw}_{\mathrm{H}}\right)_{\downarrow}$.

By using QTAIM analysis it was identified that $\mathrm{H}, \mathrm{rH}, \mathrm{w}_{\mathrm{H}}$ and $\mathrm{rw}_{\mathrm{H}}$ conformers of the $\mathrm{G} \cdot \mathrm{C}$ nucleobase pair are bounded by the $\mathrm{CH} \cdots \mathrm{N}, \mathrm{NH} \cdots \mathrm{C}, \mathrm{NH} \cdots \mathrm{O}, \mathrm{NH} \cdots \mathrm{N}, \mathrm{OH} \cdots \mathrm{N}$ and $\mathrm{OH} \cdots \mathrm{O}$ H-bonds.

Among the $\mathrm{G} \cdot \mathrm{C}$ nucleobase pairs in $\mathrm{H}, \mathrm{rH}, \mathrm{w}_{\mathrm{H}}$ and $\mathrm{rw}_{\mathrm{H}}$ configurations the following complexes have significantly nonplanar geometry: $\quad \mathrm{G}^{* \mathrm{t}} \cdot \mathrm{C}^{*}{ }_{\mathrm{O} 2}\left(\mathrm{w}_{\mathrm{H}}\right)_{\uparrow}, \quad \mathrm{G}^{* \mathrm{t}} \cdot \mathrm{C}^{*}{ }_{\mathrm{O} 2}\left(\mathrm{rw}_{\mathrm{WC} / \mathrm{H}}\right)$, $\mathrm{G}^{* \mathrm{t}} \cdot \mathrm{C}^{* \mathrm{t}}{ }_{\mathrm{O} 2}\left(\mathrm{rw}_{\mathrm{H}}\right)_{\uparrow}, \mathrm{G}^{* \mathrm{t}} \cdot \mathrm{C}^{* \mathrm{t}}{ }_{\mathrm{O} 2}\left(\mathrm{w}_{\mathrm{H}}\right)_{\downarrow}, \mathrm{G}^{* \mathrm{t}} \cdot \mathrm{C}\left(\mathrm{rw}_{\mathrm{H}}\right), \mathrm{G}^{* \mathrm{t}} \cdot \mathrm{C}\left(\mathrm{rw}_{\mathrm{H}}\right)_{\uparrow}$, $\mathrm{G}^{* \mathrm{t}} \cdot \mathrm{C}\left(\mathrm{w}_{\mathrm{H}}\right)_{\uparrow}$ and $\mathrm{G}^{*}{ }_{\mathrm{N} 7} \cdot \mathrm{C}^{* \mathrm{t}}\left(\mathrm{rw}_{\mathrm{H}}\right)_{\uparrow}$. This non-planarity of the 
complexes is also caused by the non-planarity of the $\mathrm{NH}_{2}$ amino groups of the $\mathrm{G}$ and $\mathrm{C}$ nucleobases.

13. It is especially interesting to note the cases, when rotation of the bases within the $\mathrm{G} \cdot \mathrm{C}$ nucleobase pairs leads to the changing of the geometry from Watson-Crick to Hoogsteen and vice versa through the intermediate wobble $\left(\mathrm{w}_{\mathrm{WC} / \mathrm{H}}\right)$ or reverse wobble $\left(\mathrm{rw}_{\mathrm{WC} / \mathrm{H}}\right)$ base pairs via the $\mathrm{G}^{*} \cdot \mathrm{C}^{*}(\mathrm{WC}) \leftrightarrow \mathrm{G}^{*} \cdot \mathrm{C}^{*}\left(\mathrm{rw}_{\mathrm{WC} /}\right.$ $\mathrm{H}), \quad \mathrm{G}^{*} \cdot \mathrm{C}^{*}(\mathrm{rWC}) \leftrightarrow \mathrm{G}^{*} \cdot \mathrm{C}^{*}\left(\mathrm{w}_{\mathrm{WC} / \mathrm{H}}\right)$ and $\mathrm{G}^{* \mathrm{t}} \cdot \mathrm{C}^{*}(\mathrm{rH}) \leftrightarrow$ $\mathrm{G}^{* \mathrm{t}} \cdot \mathrm{C}^{*}\left(\mathrm{~W}_{\mathrm{WC} / \mathrm{H}}\right)$ conformational transformations. This became possible due to the transformations of the Watson-Crick $\mathrm{G} \cdot \mathrm{C}(\mathrm{WC})$, reverse Watson-Crick $\mathrm{G} \cdot \mathrm{C}(\mathrm{rWC})$ and reverse Hoogsteen $\mathrm{G} \cdot \mathrm{C}(\mathrm{rH})$ base pairs into their tautomerised states $\mathrm{G}^{*} \cdot \mathrm{C}^{*}(\mathrm{WC}), \mathrm{G}^{*} \cdot \mathrm{C}^{*}(\mathrm{rWC})$ and $\mathrm{G}^{* \mathrm{t}} \cdot \mathrm{C}^{*}(\mathrm{rH})$, respectively.

14. All TSs of the conformational transformations are stabilized by specific intermolecular contacts - H-bonds and attractive van der Waals contacts (from 1 to 3 ). In the most variety of cases TSs of the conformational transformations are joined by single

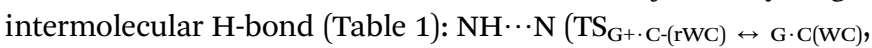
$\mathrm{TS}_{\mathrm{G}^{*} \cdot \mathrm{C}^{*}(\mathrm{WC}) \leftrightarrow \mathrm{G}^{*} \cdot \mathrm{C}^{*}(\mathrm{rWC})}, \mathrm{TS}_{\mathrm{G}^{*} \cdot \mathrm{C}^{*}(\mathrm{rWC}) \leftrightarrow \mathrm{G}^{*} \mathrm{t} \cdot \mathrm{C}^{*}(\mathrm{WC})}, \mathrm{TS}_{\mathrm{G}^{*} \mathrm{t} \cdot \mathrm{C}^{*} \mathrm{t}(\mathrm{WC})}$

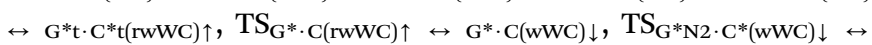

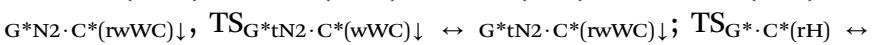
$\mathrm{G}^{*} \cdot \mathrm{C}^{*}(\mathrm{H}), \mathrm{TS}_{\mathrm{G} * \mathrm{t} \cdot \mathrm{C} *(\mathrm{H}) \leftrightarrow \mathrm{G}^{*} \mathrm{t} \cdot \mathrm{C} *(\mathrm{rH}), \mathrm{TS}_{\mathrm{G}} * \mathrm{t} \cdot \mathrm{C}(\mathrm{rwH}) \uparrow \leftrightarrow \mathrm{G} * \mathrm{t} \cdot \mathrm{C}(\mathrm{wH}) \downarrow}, \mathrm{TS}_{\mathrm{G}^{*} \mathrm{O} /}$

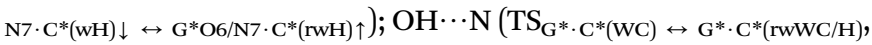

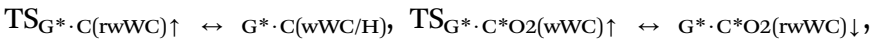

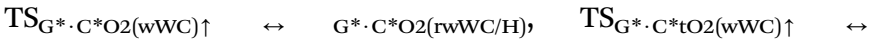
$\left.\mathrm{G}^{*} \cdot \mathrm{C}^{*} \mathrm{tO} 2(\mathrm{rwWC} / \mathrm{H}), \quad \mathrm{TS}_{\mathrm{G}^{*} \cdot \mathrm{C}^{*} \mathrm{tO} 2(\mathrm{wWC}) \uparrow} \leftrightarrow \mathrm{G}^{*} \cdot \mathrm{C}^{*} \mathrm{tO} 2(\mathrm{rwWC}) \downarrow\right) ; \quad \mathrm{OH} \cdots \mathrm{O}$ $\left(\mathrm{TS}_{\mathrm{G}^{*} \cdot \mathrm{C}^{*}(\mathrm{rWC}) \leftrightarrow \mathrm{G}^{*} \cdot \mathrm{C}^{*}(\mathrm{wWC} / \mathrm{H})}\right) ; \mathrm{NH} \cdots \mathrm{O}\left(\mathrm{TS}_{\mathrm{G}} \cdot \mathrm{C}^{*}(\mathrm{rwWC}) \uparrow \leftrightarrow \mathrm{G} \cdot \mathrm{C}^{*}(\mathrm{wWC}) \uparrow ;\right.$

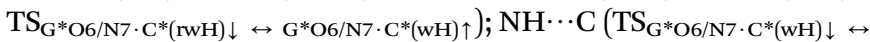

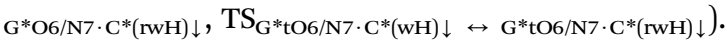

At the same time, there are also cases of the simultaneous coexistence of the two $\mathrm{H}$-bonds at the TSs $(\mathrm{NH} \cdots \mathrm{N}, \mathrm{NH} \cdots \mathrm{O}, \mathrm{OH} \cdots$ $\mathrm{N}, \mathrm{OH} \cdots \mathrm{O}$ and $\left.\mathrm{CH}^{\cdots} \cdot \mathrm{N}\right)$ (Table 1): $\mathrm{TS}_{\mathrm{G} * \mathrm{t} \cdot \mathrm{C} * \mathrm{O} 2(\mathrm{wH}) \uparrow \leftrightarrow \mathrm{G}^{*} \mathrm{t} \cdot \mathrm{C}^{*} \mathrm{O} 2(\mathrm{rwH}) \downarrow}$, $\mathrm{TS}_{\mathrm{G}^{*} \mathrm{t} \cdot \mathrm{C}^{*} \mathrm{tO} 2(\mathrm{wH}) \uparrow \leftrightarrow \mathrm{G}^{*} \mathrm{t} \cdot \mathrm{C}^{*} \mathrm{tO} 2(\mathrm{rwH}) \downarrow}, \mathrm{TS}_{\mathrm{G}^{*} \mathrm{t} \cdot \mathrm{C}^{*} \mathrm{tO} 2(\mathrm{rwH}) \downarrow \leftrightarrow \mathrm{G}^{*} \mathrm{t} \cdot \mathrm{C}^{*} \mathrm{tO} 2(\mathrm{wH}) \downarrow}$, $\mathrm{TS}_{\mathrm{G} * \mathrm{~N} 7 \cdot \mathrm{C}^{*}(\mathrm{rwH}) \uparrow \leftrightarrow \mathrm{G}^{*} \mathrm{~N} 7 \cdot \mathrm{C}^{*}(\mathrm{wH}) \uparrow}$ and $\mathrm{TS}_{\mathrm{G}} * \mathrm{O} 6 / \mathrm{N} 7 \cdot \mathrm{C}^{*}(\mathrm{rwH}) \uparrow \leftrightarrow \mathrm{G}^{*} \mathrm{O} 6 /$ $\mathrm{N} 7 \cdot \mathrm{C}^{*}(\mathrm{wH}) \uparrow$, especially those involving $\mathrm{NH}_{2}$ amino groups of the $\mathrm{G}$

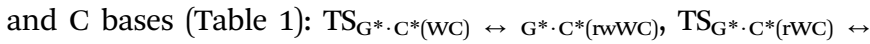

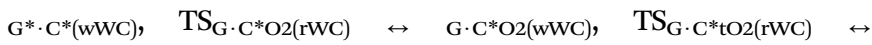
G.C.tO2(wWC), $\quad \mathrm{TS}_{\mathrm{G}^{*} \mathrm{t} \cdot \mathrm{C}^{*} \mathrm{t}(\mathrm{WC}) \quad \leftrightarrow \quad \mathrm{G}^{*} \mathrm{t} \cdot \mathrm{C}^{*} \mathrm{t}(\mathrm{rwWC}),} \mathrm{TS}_{\mathrm{G} \cdot \mathrm{C}^{*}(\mathrm{wWC}) \uparrow} \leftrightarrow$

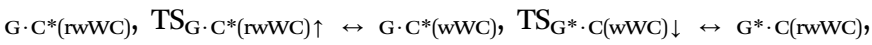
$\mathrm{TS}_{\mathrm{G}^{*} \cdot \mathrm{C}^{*} \mathrm{O} 2(\mathrm{rwWC}) \downarrow \leftrightarrow \mathrm{G}^{*} \cdot \mathrm{C}^{*} \mathrm{O} 2(\mathrm{wWC})}, \mathrm{TS}_{\mathrm{G}^{*} \mathrm{t} \cdot \mathrm{C}^{*} \mathrm{O} 2(\mathrm{rwWC}) \downarrow \leftrightarrow \mathrm{G}^{*} \mathrm{t} \cdot \mathrm{C}^{*} \mathrm{O} 2(\mathrm{wWC})}$, $\mathrm{TS}_{\mathrm{G}^{*} \cdot \mathrm{C}^{*} \mathrm{tO} 2(\mathrm{rwWC}) \downarrow} \leftrightarrow \quad \mathrm{G}^{*} \cdot \mathrm{C} * \mathrm{tO} 2(\mathrm{wWC}), \quad \mathrm{TS}_{\mathrm{G}} * \mathrm{t} \cdot \mathrm{C}^{*} \mathrm{tO} 2(\mathrm{rwWC}) \downarrow \quad \leftrightarrow$ $\mathrm{G}^{*} \mathrm{t} \cdot \mathrm{C}^{*} \mathrm{tO} 2(\mathrm{wWC}) ; \mathrm{TS}_{\mathrm{G}} * \mathrm{t} \cdot \mathrm{C}(\mathrm{wH}) \downarrow \leftrightarrow \mathrm{G}^{*} \cdot \mathrm{C}(\mathrm{rwH})$.

Especially interesting are the cases of the following TSs $\mathrm{TS}_{\mathrm{G} \cdot \mathrm{C}^{*} \mathrm{O} 2(\mathrm{rWC}) \leftrightarrow \mathrm{G} \cdot \mathrm{C}^{*} \mathrm{O} 2(\mathrm{wWC})}, \mathrm{TS}_{\mathrm{G} \cdot \mathrm{C}^{*} \mathrm{tO} 2(\mathrm{rWC}) \leftrightarrow \mathrm{G} \cdot \mathrm{C}^{*} \mathrm{tO} 2(\mathrm{wWC})}$, $\mathrm{TS}_{\mathrm{G}} \cdot \mathrm{C}^{*}(\mathrm{wWC}) \uparrow \leftrightarrow \mathrm{G} \cdot \mathrm{C}^{*}(\mathrm{rwWC}), \mathrm{TS}_{\mathrm{G}} \cdot \mathrm{C}^{*}(\mathrm{rwWC}) \uparrow \leftrightarrow \mathrm{G} \cdot \mathrm{C}^{*}(\mathrm{wWC})$, where the $\mathrm{N} 1 \mathrm{H} \cdots \mathrm{N} 3$ and $\mathrm{N} 2 \mathrm{H} \cdots \mathrm{N} 3 / \mathrm{N} 1 \mathrm{H} \cdots \mathrm{N} 3$ and $\mathrm{N} 2 \mathrm{H} \cdots \mathrm{N} 3 / \mathrm{N} 1 \mathrm{H} \cdots \mathrm{O} 2$ and $\mathrm{N} 2 \mathrm{H} \cdots \mathrm{O} 2 / \mathrm{N} 1 \mathrm{H} \cdots \mathrm{N} 4$ and $\mathrm{N} 2 \mathrm{H} \cdots \mathrm{N} 4 \mathrm{H}$-bonds are focused on one common $\mathrm{N} 3 / \mathrm{N} 3 / \mathrm{O} 2 / \mathrm{N} 4$ atom, respectively.

Also, there are cases of the H-bonds ( $\mathrm{NH} \cdots \mathrm{O}, \mathrm{NH} \cdots \mathrm{N}$ and $\mathrm{OH} \cdots \mathrm{N})$, which are combined with attractive van der Waals

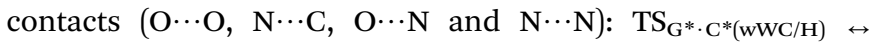

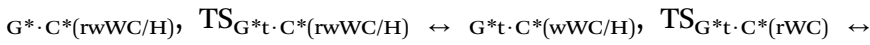
$\mathrm{G}^{*} \cdot \mathrm{C}^{*}(\mathrm{wWC}), \quad \mathrm{TS}_{\mathrm{G} \cdot \mathrm{C}^{*}(\mathrm{rwWC})} \leftrightarrow \mathrm{G} \cdot \mathrm{C}^{*}(\mathrm{wWC}), \quad \mathrm{TS}_{\mathrm{G}^{*} \mathrm{tN} 2 \cdot \mathrm{C}^{*}(\mathrm{rwWC}) \downarrow} \leftrightarrow$

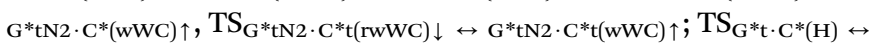

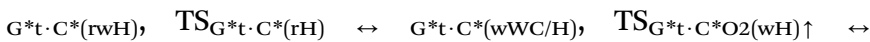
$\mathrm{G}^{*} \mathrm{t} \cdot \mathrm{C}^{*} \mathrm{O} 2(\mathrm{rwH}), \mathrm{TS}_{\mathrm{G}}{ }^{*} \cdot \mathrm{C}^{*} \mathrm{O} 2(\mathrm{wH}) \uparrow \leftrightarrow \mathrm{G}^{*} \cdot \mathrm{C}^{*} \mathrm{O} 2(\mathrm{rwWC} / \mathrm{H}), \mathrm{TS}_{\mathrm{G}}{ }^{*} \cdot \mathrm{C}^{*} \mathrm{tO} 2(\mathrm{wH}) \uparrow$ $\leftrightarrow \mathrm{G}^{*} \mathrm{t} \cdot \mathrm{C} \cdot \mathrm{tO} 2(\mathrm{rwH}) \uparrow, \mathrm{TS}_{\mathrm{G}} * \mathrm{t} \cdot \mathrm{C}(\mathrm{rwH}) \uparrow \leftrightarrow \mathrm{G}^{*} \mathrm{t} \cdot \mathrm{C}(\mathrm{wH}) \uparrow, \mathrm{TS}_{\mathrm{G}} * \mathrm{~N} 7 \cdot \mathrm{C} *(\mathrm{rwH}) \uparrow \leftrightarrow$ $\mathrm{G}^{*} \mathrm{~N} 7 \cdot \mathrm{C}^{*}(\mathrm{wH}) \cdot$

Finally, there are also TSs stabilized by three intermolecular

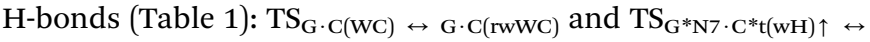
$\mathrm{G}^{*} \mathrm{~N} 7 \cdot \mathrm{C}^{*} \mathrm{t}(\mathrm{rwH}) \uparrow \cdot$

It is especially interesting to note the cases of the TSs $\mathrm{TS}_{\mathrm{G} * \mathrm{t} \cdot \mathrm{C} *(\mathrm{H}) \quad \leftrightarrow \quad \mathrm{G}^{*} \cdot \mathrm{C}^{*}(\mathrm{rwH}),} \mathrm{TS}_{\mathrm{G} * \mathrm{t} \cdot \mathrm{C} *(\mathrm{rH})} \leftrightarrow \quad \mathrm{G}^{*} \cdot \mathrm{C}^{*}(\mathrm{wWC} / \mathrm{H})$, $\mathrm{TS}_{\mathrm{G}} *_{\mathrm{t}} \cdot \mathrm{C}^{*} \mathrm{O} 2(\mathrm{wH}) \uparrow \leftrightarrow \mathrm{G}^{*} \cdot \mathrm{C} \cdot \mathrm{O} 2(\mathrm{rwH}), \mathrm{TS}_{\mathrm{G}}{ }^{*} \cdot \mathrm{C}^{*} \mathrm{O} 2(\mathrm{wH}) \uparrow \leftrightarrow \mathrm{G}^{*} \cdot \mathrm{C}^{*} \mathrm{O} 2(\mathrm{rwWC} / \mathrm{H})$, $\mathrm{TS}_{\mathrm{G}} *_{\mathrm{t}} \cdot \mathrm{C}^{*} \mathrm{tO} 2(\mathrm{wH}) \uparrow \leftrightarrow \mathrm{G}^{*} \cdot \mathrm{C}^{*} \mathrm{tO} 2(\mathrm{rwH}) \uparrow, \mathrm{TS}_{\mathrm{G}} *_{\mathrm{t}} \cdot \mathrm{C}(\mathrm{rwH}) \uparrow \leftrightarrow \mathrm{G}^{*} \mathrm{t} \cdot \mathrm{C}(\mathrm{wH}) \uparrow$,

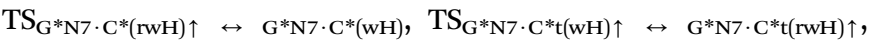
where the $\mathrm{O} 6 \mathrm{H} \cdots \mathrm{N} 4$ and $\mathrm{N} 7 \cdots \mathrm{N} 4 / \mathrm{O} 6 \mathrm{H} \cdots \mathrm{O} 2$ and $\mathrm{N} 7 \cdots \mathrm{O} 2 / \mathrm{O} 6 \mathrm{H} \cdots$ $\mathrm{N} 3$ and $\mathrm{N} 7 \cdots \mathrm{N} 3 / \mathrm{O} 6 \mathrm{H} \cdots \mathrm{N} 3$ and $\mathrm{N} 7 \cdots \mathrm{N} 3 / \mathrm{O} 6 \mathrm{H} \cdots \mathrm{N} 3$ and $\mathrm{N} 7 \cdots \mathrm{N} 3 /$ $\mathrm{O} 6 \mathrm{H} \cdots \mathrm{N} 3$ and $\mathrm{N} 7 \cdots \mathrm{N} 3 / \mathrm{O} 6 \cdots \mathrm{N} 4$ and $\mathrm{N} 7 \mathrm{H} \cdots \mathrm{N} 4 / \mathrm{N} 4 \mathrm{H} \cdots \mathrm{O} 6$ and $\mathrm{N} 3 \mathrm{H} \cdots \mathrm{O} 6 \mathrm{H}$-bonds and attractive van der Waals contacts are focused on one common N4/O2/N3/N3/N3/N3/N4/O6 atom, respectively.

15. Moreover, careful analysis of the specificities of the geometrical structures of the TSs shows that the term "rotation around the individual intermolecular H-bond" should not be considered literally, in the sense of the rotation around some imaginary non-deformable fixed axis, but in the sense of the rotation around labile "axis" of rotation. Notably, that exactly this circumstance significantly complicates the procedure of the localization of the corresponding transition states, which to some extent could be considered as an art. Localization of the TSs is also complicated by the fact that corresponding hypersurface of the electronic energy is quite diverse. From our point of view, some results evidence about this, when rotations around one and the same H-bond, which of course are controlled by the different TSs, lead to the different structural consequences, that is to the different conformers of the $\mathrm{G} \cdot \mathrm{C}$ nucleobase pair.

16. Investigated conformational transformations are dipole active, for which the dipole moments of the TSs change in the wide range of values: $\mu=1.08-10.57 / 1.45-9.89 \mathrm{D}$ (Table 1). At this, in the vast majority of cases, the dipole moments of the TSs are less than dipole moments of the starting and final structures (Table 1).

17. Especial interest attracts conformational transformations in the $\mathrm{G} \cdot \mathrm{C}\left(\mathrm{w}_{\mathrm{H}}\right) / \mathrm{G} \cdot \mathrm{C}\left(\mathrm{rw}_{\mathrm{H}}\right)$ base pairs, where $\mathrm{G}$ is in the yilidic tautomeric form. In all these cases, conformational degree of freedom ensures the unusual intermolecular $\mathrm{C} 8 \cdots \mathrm{HN} \mathrm{H}$-bond: $\mathrm{G}^{*}{ }_{\mathrm{O} 6 / \mathrm{N} 7} \cdot \mathrm{C}^{*}\left(\mathrm{w}_{\mathrm{H}}\right)_{\downarrow} \leftrightarrow \mathrm{G}^{*}{ }_{\mathrm{O} / \mathrm{N} 7} \cdot \mathrm{C}^{*}\left(\mathrm{rw}_{\mathrm{H}}\right)_{\downarrow}, \mathrm{G}^{*}{ }_{\mathrm{O} 6 / \mathrm{N} 7} \cdot \mathrm{C}^{*}\left(\mathrm{w}_{\mathrm{H}}\right)_{\downarrow} \leftrightarrow$ $\mathrm{G}^{*}{ }_{\mathrm{O} / \mathrm{N} 7} \cdot \mathrm{C}^{*}\left(\mathrm{rw}_{\mathrm{H}}\right)_{\uparrow}, \mathrm{G}^{*}{ }_{\mathrm{O} 6 / \mathrm{N} 7} \cdot \mathrm{C}^{*}\left(\mathrm{rw}_{\mathrm{H}}\right)_{\downarrow} \leftrightarrow \mathrm{G}^{*}{ }_{\mathrm{O} 6 / \mathrm{N} 7} \cdot \mathrm{C}^{*}\left(\mathrm{w}_{\mathrm{H}}\right)_{\uparrow}$ and $\mathrm{G}^{* \mathrm{t}}{ }_{\mathrm{O} 6 / \mathrm{N} 7} \cdot \mathrm{C}^{*}\left(\mathrm{w}_{\mathrm{H}}\right)_{\downarrow} \leftrightarrow \mathrm{G}^{* \mathrm{t}}{ }_{\mathrm{O} 6 / \mathrm{N} 7} \cdot \mathrm{C}^{*}\left(\mathrm{rw}_{\mathrm{H}}\right)_{\downarrow}$ (Table 1).

\section{Conclusions}

By the methods of quantum chemistry at the MP2/6$311++\mathrm{G}(2 \mathrm{df}, \mathrm{pd}) / / \mathrm{B} 3 \mathrm{LYP} / 6-311++\mathrm{G}(\mathrm{d}, \mathrm{p})$ level of theory, it was investigated in detail conformational possibilities of the isolated $\mathrm{G} \cdot \mathrm{C}$ nucleotide base pairs by the participation of the $\mathrm{G}$ and $\mathrm{C}$ bases in the canonical and rare tautomeric forms.

Provided investigation of the conformational transformations of the biologically important $\mathrm{G} \cdot \mathrm{C}$ (WC, rWC, $\mathrm{w}_{\mathrm{WC}}$, $\mathrm{rw}_{\mathrm{WC}}, \mathrm{H}, \mathrm{rH}, \mathrm{w}_{\mathrm{H}}$ and $\mathrm{rw}_{\mathrm{H}}$ ) nucleobase pairs, which is caused by the rotational mobility of the bases around the individual 
intermolecular $\mathrm{H}$-bonds, significantly extends the existing pull of the unusual conformers of the $\mathrm{G} \cdot \mathrm{C}$ base pairs and thoughts about the microstructural mechanisms of these processes, as well as about their functional role.

As a result of this investigation, we have revealed a wide set of the surprising conformers-rotamers of the $\mathrm{G} \cdot \mathrm{C}$ nucleobase pairs, which could be incorporated into the double helix of the parallel or anti-parallel ${ }^{50}$ DNA and RNA molecules. High-energy conformers of the $\mathrm{G} \cdot \mathrm{C}$ nucleobase pair, formed by the interbonded non-dissociative conformational transformations, most likely in our opinion play the outstanding role in support of the unique spatial structure of the nucleic acids, especially of the RNA molecule, and their functionally-important rebuildings, which are usually caused by the proteins.

And finally, it deserves to pay especial attention to the fact that localization of the TSs, describing the conformational mobility of the $\mathrm{G} \cdot \mathrm{C}$ nucleobase pairs, is quite delicate procedure in its essence, approaching to the art. So, we hope that presented here results would simplify further work in this biologically-perspective direction.

\section{Conflicts of interest}

There are no conflicts to declare.

\section{References}

1 O. O. Brovarets', K. S. Tsiupa and D. M. Hovorun, Where quantum biochemistry meets structural bioinformatics: Excited conformationally-tautomeric states of the classical A.T DNA base pair, in DNA - Damages and Repair Mechanisms, ed. P. Behzadi, InTechOpen, London, UK, May 2021, DOI: 10.5772/intechopen.94565.

2 J. D. Watson and F. H. C. Crick, Molecular structure of nucleic acids: A structure for deoxyribose nucleic acid, Nature, 1953, 171, 737-738.

3 J. D. Watson and F. H. C. Crick, The structure of DNA, Cold Spring Harbor Symp. Quant. Biol., 1953, 18, 123-131.

4 P. Pospisil, P. Ballmer, L. Scapozza and G. Folkers, Tautomerism in computer-aided drug design, J. Recept. Signal Transduction, 2003, 23, 361-371.

5 A. J. Cruz-Cabeza and C. R. Groom, Identification, classification and relative stability of tautomers in the cambridge structural database, CrystEngComm, 2011, 13, 93-98.

6 D. K. Dhaked, L. Guash and M. C. Nicklaus, Tautomer database: A comprehensive resource for tautomerism analyses, J. Chem. Inf. Model., 2020, 60, 1090-1100.

7 O. Wahl and T. T. Sander, An open tautomer database, $J$. Chem. Inf. Model., 2020, 60, 1085-1089.

8 D. K. Dhaked, W.-D. Ihlenfeldt, H. Patel, V. Delannee and M. C. Nicklaus, Toward a comprehensive treatment of tautomerism in chemoinformatics including in InChI V2, J. Chem. Inf. Model., 2020, 60, 1253-1275.

9 O. O. Brovarets', R. O. Zhurakivsky and D. M. Hovorun, DPT tautomerization of the long $\mathrm{A} \cdot \mathrm{A}^{*}$ Watson-Crick base pair formed by the amino and imino tautomers of adenine:
Combined QM and QTAIM investigation, J. Mol. Model., 2013, 19, 4223-4237.

10 O. O. Brovarets', I. M. Kolomiets' and D. M. Hovorun, Elementary molecular mechanisms of the spontaneous point mutations in DNA: A novel quantum-chemical insight into the classical understanding, Quantum chemistry - molecules for innovations, ed. T. Tada, Rijeka: In Tech Open Access, 2012.

11 O. O. Brovarets', R. O. Zhurakivsky and D. M. Hovorun, A QM/QTAIM microstructural analysis of the tautomerisation via the DPT of the hypoxanthine-adenine nucleobase pair, Mol. Phys., 2014, 112, 2005-2016.

12 O. O. Brovarets' and D. M. Hovorun, The nature of the transition mismatches with Watson-Crick architecture: The $\mathrm{G}^{*} \cdot \mathrm{T}$ or $\mathrm{G} \cdot \mathrm{T}^{*}$ DNA base mispair or both? A $\mathrm{QM} /$ QTAIM perspective for the biological problem, J. Biomol. Struct. Dyn., 2015, 33, 925-945.

13 O. O. Brovarets' and D. M. Hovorun, IR Vibrational spectra of H-bonded complexes of adenine, 2-aminopurine and 2aminopurine $^{+}$with cytosine and thymine: Quantumchemical study, Opt. Spectrosc., 2011, 111, 750-757.

14 J. S. Kwiatkowski, T. Julia and Z. R. Rein, Quantummechanical prediction of tautomeric equilibria, $A d v$. Quantum Chem., 1986, 18, 85-130.

15 H. A. Heus and C. W. Hilbers, Structures of non-canonical tandem base pairs in RNA helices: Review, Nucleosides, Nucleotides Nucleic Acids, 2003, 22, 559-571.

16 S. Mukherjee, M. Bansal and D. Bhattacharyya, Conformational specificity of non-canonical base pairs and higher order structures in nucleic acids: Crystal structure database analysis, J. Comput.-Aided Mol. Des., 2006, 20, 629-645.

17 J. Das, S. Mukherjee, A. Mitra and D. Bhattacharyya, Noncanonical base pairs and higher order structures in nucleic acids: Crystal structure database analysis, J. Biomol. Struct. Dyn., 2006, 24, 149-161.

18 P. Sharma, A. Mitra, S. Sharma, H. Singh and D. Bhattacharyya, Quantum chemical studies of structures and binding in noncanonical RNA base pairs: The trans Watson-Crick:Watson-Crick family, J. Biomol. Struct. Dyn., 2008, 25, 709-732.

19 S. Halder and D. Bhattacharyya, RNA structure and dynamics: A base pairing perspective, Prog. Biophys. Mol. Biol., 2013, 113, 264-283.

20 S. Bhattacharya, A. Jhunjhunwala, A. Halder, D. Bhattacharyya and A. Mitra, Going beyond base-pairs: Topology-based characterization of base-multiplets in RNA, RNA, 2019, 25, 573-589.

21 O. O. Brovarets' and D. M. Hovorun, How stable are mutagenic tautomers of the DNA bases?, Biopolym. Cell, 2010, 26, 72-76.

22 O. O. Brovarets' and D. M. Hovorun, Stability of mutagenic tautomers of uracil and its halogen derivatives: The results of quantum-mechanical investigation, Biopolym. Cell, 2010, 26, 295-298.

23 O. O. Brovarets' and D. M. Hovorun, Intramolecular tautomerization and the conformational variability of 
some classical mutagens - cytosine derivatives: Quantum chemical study, Biopolym. Cell, 2011, 27, 221-230.

$24 \mathrm{H}$. Zheng, D. Zhao and Z. Yang, Theoretical study of the intramolecular proton transfer in the tautomers of cytosine assisted by water, Chin. J. Chem., 2011, 29, 2243-2248.

25 F. H. C. Crick and J. D. Watson, The complementary structure of deoxyribonucleic acid, Proc. R. Soc., 1954, 223, 80-96.

26 P.-O. Löwdin, Proton tunneling in DNA and its biological implications, Rev. Mod. Phys., 1963, 35, 724-732.

27 P.-O. Löwdin, Quantum genetics and the aperiodic solid: Some aspects on the biological problems of heredity, mutations, aging, and tumors in view of the quantum theory of the DNA molecule, in Advances in Quantum Chemistry, ed. P.-O. Löwdin, New York, NY; London, Academic Press, 1966, pp. 213-360.

28 O. O. Brovarets' and D. M. Hovorun, Can tautomerization of the A·T Watson-Crick base pair via double proton transfer provoke point mutations during DNA replication? A comprehensive QM and QTAIM analysis, J. Biomol. Struct. Dyn., 2014, 32, 127-154.

29 O. O. Brovarets' and D. M. Hovorun, Proton tunneling in the A $\cdot$ T Watson-Crick DNA base pair: Myth or reality?, J. Biomol. Struct. Dyn., 2015, 33, 2716-2720.

30 O. O. Brovarets' and D. M. Hovorun, Atomistic mechanisms of the double proton transfer in the H-bonded nucleobase pairs: QM/QTAIM computational lessons, J. Biomol. Struct. Dyn., 2018, 37, 1880-1907.

31 O. O. Brovarets' and D. M. Hovorun, Why the tautomerization of the G.C Watson-Crick base pair via the DPT does not cause point mutations during DNA replication? QM and QTAIM comprehensive analysis, J. Biomol. Struct. Dyn., 2014, 32, 1474-1499.

32 B. Bezbaruah and B. C. Medhi, Quantum mechanical study on the proton transfer mechanism within adenine-thymine and guanine-cytosine base pairs of DNA nucleobase, Indian J. Adv. Chem. Sci., 2016, 4, 314-320.

33 A. Shekaari and M. Jafari, Modeling the action of environment on proton tunneling in the adenine-thymine base pair, Prog. Biophys. Mol. Biol., 2020, 150, 98-103.

34 O. O. Brovarets', I. S. Voiteshenko, H. E. Perez-Sanchez and D. M. Hovorun, A QM/QTAIM research under the magnifying glass of the DPT tautomerisation of the wobble mispairs involving 2-aminopurine, New J. Chem., 2017, 41, 7232-7243.

35 O. O. Brovarets', I. S. Voiteshenko and D. M. Hovorun, Physico-chemical profiles of the wobble $\leftrightarrow$ Watson-Crick $\mathrm{G}^{*} \cdot 2 \mathrm{AP}(\mathrm{w}) \leftrightarrow \mathrm{G} \cdot 2 \mathrm{AP}(\mathrm{WC})$ and $\mathrm{A} \cdot 2 \mathrm{AP}(\mathrm{w}) \leftrightarrow \mathrm{A}^{*} \cdot 2 \mathrm{AP}(\mathrm{WC})$ tautomerisations: A QM/QTAIM comprehensive survey, Phys. Chem. Chem. Phys., 2018, 20, 623-636.

36 O. O. Brovarets' and D. M. Hovorun, Renaissance of the tautomeric hypothesis of the spontaneous point mutations in DNA: New ideas and computational approaches. Mitochondrial DNA - New Insights, IntechOpen, ed. H. Seligmann, London, United Kingdom, 2018, ISBN 978-95351-6167-7.
37 O. O. Brovarets' and D. M. Hovorun, How many tautomerization pathways connect Watson-Crick-like $\mathrm{G}^{*} \cdot \mathrm{T}$ DNA base mispair and wobble mismatches?, J. Biomol. Struct. Dyn., 2015, 33, 2297-2315.

38 O. O. Brovarets' and D. M. Hovorun, Novel physico-chemical mechanism of the mutagenic tautomerisation of the Watson-Crick-like A.G and C.T DNA base mispairs: A quantum-chemical picture, $R S C$ Adv., 2015, 5, 66318-66333.

39 O. O. Brovarets' and D. M. Hovorun, Tautomeric transition between wobble A.C DNA base mispair and Watson-Cricklike A. $\mathrm{C}^{*}$ mismatch: Microstructural mechanism and biological significance, Phys. Chem. Chem. Phys., 2015, 17, 15103-15110.

40 O. O. Brovarets' and D. M. Hovorun, Wobble $\leftrightarrow$ WatsonCrick tautomeric transitions in the homo-purine DNA mismatches: A key to the intimate mechanisms of the spontaneous transversions, J. Biomol. Struct. Dyn., 2015, 33, 2710-2715.

41 O. O. Brovarets' and D. M. Hovorun, A novel conception for spontaneous transversions caused by homo-pyrimidine DNA mismatches: A QM/QTAIM highlight, Phys. Chem. Chem. Phys., 2015, 17, 21381-21388.

42 O. O. Brovarets' and D. M. Hovorun, New structural hypostases of the A.T and G.C Watson-Crick DNA base pairs caused by their mutagenic tautomerisation in a wobble manner: A QM/QTAIM prediction, RSC Adv., 2015, 5, 99594-99605.

43 O. O. Brovarets', K. S. Tsiupa and D. M. Hovorun, Novel pathway for mutagenic tautomerization of classical A.T DNA base pairs via sequential proton transfer through quasi-orthogonal transition states: A QM/QTAIM investigation, PLOS One, 2018, 13, e0199044.

44 O. O. Brovarets', K. S. Tsiupa and D. M. Hovorun, Unexpected $\mathrm{A} \cdot \mathrm{T}(\mathrm{WC}) \leftrightarrow \mathrm{A} \cdot \mathrm{T}(\mathrm{rWC}) / \mathrm{A} \cdot \mathrm{T}(\mathrm{rH})$ and $\mathrm{A} \cdot \mathrm{T}(\mathrm{H}) \leftrightarrow$ $\mathrm{A} \cdot \mathrm{T}(\mathrm{rH}) / \mathrm{A} \cdot \mathrm{T}(\mathrm{rWC})$ conformational transitions between the classical A $\cdot T$ DNA base pairs: A QM/QTAIM comprehensive study, Int. J. Quantum Chem., 2018, 118, e25674.

45 O. O. Brovarets', K. S. Tsiupa and D. M. Hovorun, Nondissociative structural transitions of the Watson-Crick and reverse Watson-Crick A.T DNA base pairs into the Hoogsteen and reverse Hoogsteen forms, Sci. Repts., 2018, 8, 10371.

46 O. O. Brovarets', K. S. Tsiupa and D. M. Hovorun, The $\mathrm{A} \cdot \mathrm{T}(\mathrm{rWC}) / \mathrm{A} \cdot \mathrm{T}(\mathrm{H}) / \mathrm{A} \cdot \mathrm{T}(\mathrm{rH}) \quad \leftrightarrow \quad \mathrm{A} \cdot \mathrm{T}^{*}\left(\mathrm{rw}_{\mathrm{WC}}\right) / \mathrm{A} \cdot \mathrm{T}^{*}\left(\mathrm{w}_{\mathrm{H}}\right) /$ $\mathrm{A} \cdot \mathrm{T} *\left(\mathrm{rw}_{\mathrm{H}}\right)$ mutagenic tautomerization via sequential proton transfer: A QM/QTAIM study, RSC Adv., 2018, 8, 13433-13445.

47 O. O. Brovarets', K. S. Tsiupa, A. Dinets and D. M. Hovorun, Unexpected routes of the mutagenic tautomerization of the T nucleobase in the classical A.T DNA base pairs: A QM/ QTAIM comprehensive view, Front. Chem., 2018, 6, 532.

48 O. O. Brovarets', T. A. Oliynyk and D. M. Hovorun, Novel tautomerisation mechanisms of the biologically important conformers of the reverse Löwdin, Hoogsteen, and reverse Hoogsteen $\mathrm{G}^{*} \cdot \mathrm{C}^{*}$ DNA base pairs via proton transfer: A quantum-mechanical survey, Front. Chem., 2019, 7, 597. 
49 O. O. Brovarets', A. Muradova and D. M. Hovorun, A quantum-mechanical looking behind the scene of the classical G.C nucleobase pairs tautomerization, Front. Chem., 2020, 8, 574454.

50 M. Szabat and R. Kierzek, Parallel-stranded DNA and RNA duplexes: Structural features and potential applications, FEBS J., 2017, 284, 3986-3998.

51 J. Tirado-Rives and W. L. Jorgensen, Performance of B3LYP Density Functional Methods for a large set of organic molecules, J. Chem. Theory Comput., 2008, 4, 297-306.

52 R. G. Parr and W. Yang, Density-functional theory of atoms and molecules, Oxford, Oxford University Press, 1989.

53 C. Lee, W. Yang and R. G. Parr, Development of the ColleSalvetti correlation-energy formula into a functional of the electron density, Phys. Rev. B: Condens. Matter Mater. Phys., 1988, 37, 785-789.

54 P. C. Hariharan and J. A. Pople, The influence of polarization functions on molecular orbital hydrogenation energies, Theor. Chim. Acta, 1973, 28, 213-222.

55 R. Krishnan, J. S. Binkley, R. Seeger and J. A. Pople, Self-consistent molecular orbital methods. XX. A basis set for correlated wave functions, J. Chem. Phys., 1980, 72, 650-654.

56 M. J. Frisch, G. W. Trucks, H. B. Schlegel, G. E. Scuseria, M. A. Robb, J. R. Cheeseman and J. A. Pople, GAUSSIAN 09 (Revision B.01), Wallingford CT, Gaussian Inc, 2010.

57 C. F. Matta, How dependent are molecular and atomic properties on the electronic structure method? Comparison of Hartree-Fock, DFT, and MP2 on a biologically relevant set of molecules, J. Comput. Chem., 2010, 31, 1297-1311.

58 O. O. Brovarets', R. O. Zhurakivsky and D. M. Hovorun, DPT tautomerisation of the wobble guanine thymine DNA base mispair is not mutagenic: QM and QTAIM arguments, $J$. Biomol. Struct. Dyn., 2015, 33, 674-689.
59 C. Peng, P. Y. Ayala, H. B. Schlegel and M. J. Frisch, Using redundant internal coordinates to optimize equilibrium geometries and transition states, J. Comput. Chem., 1996, 17, 49-56.

60 B. E. García-Moreno, J. J. Dwyer, A. G. Gittis, E. E. Lattman, D. S. Spencer and W. E. Stites, Experimental measurement of the effective dielectric in the hydrophobic core of a protein, Biophys. Chem., 1997, 64, 211-224.

$61 \mathrm{~S}$. T. Bayley, The dielectric properties of various solid crystalline proteins, amino acids and peptides, Trans. Faraday Soc., 1951, 47, 509-517.

62 M. J. Frisch, M. Head-Gordon and J. A. Pople, Semi-direct algorithms for the MP2 energy and gradient, Chem. Phys. Lett., 1990, 166, 281-289.

63 R. A. Kendall, T. H. Jr Dunning and R. J. Harrison, Electron affinities of the first-row atoms revisited. Systematic basis sets and wave functions, J. Chem. Phys., 1992, 96, 6796-6806.

64 R. F. W. Bader, Atoms in molecules: A quantum theory, Oxford, Oxford University Press, 1990.

65 C. F. Matta and J. Hernández-Trujillo, Bonding in polycyclic aromatic hydrocarbons in terms of the electron density and of electron delocalization, J. Phys. Chem. A, 2003, 107, 74967504.

66 C. F. Matta, N. Castillo and R. J. Boyd, Atomic contributions to bond dissociation energies in aliphatic hydrocarbons, $J$. Chem. Phys., 2006, 125, 204103.

67 I. Cukrowski and C. F. Matta, Hydrogen-hydrogen bonding: A stabilizing interaction in strained chelating rings of metal complexes in aqueous phase, Chem. Phys. Lett., 2010, 499, 66-69.

68 C. Lecomte, E. Espinosa and C. F. Matta, On atom-atom 'short contact' bonding interactions in crystals, IUCrJ, 2015, 2, 161-163.

69 T. A. Keith, AIMAll, 2010, version 10.07.01, retrieved from aim.tkgristmill.com. 